UC-20g and UC-34B

lssued: September 1986

LA--10819-T

$\mathrm{DE} 87002229$

\title{
Field Reversing Magnetotail Current Sheets: Earth, Venus, and Comet Giacobini-Zinner
}

\author{
David John McComas
}

\section{DISCLAIMER}

This report was prepared as an account of work sponsored by an agency of the United States Government. Neither the United States Goverument nor any agency thereof, nor any of their employees, makes any warranty, express or implied, or assumes any legal liability or responsibility for the accuracy, completeness, or usefulness of any information, apparatus, product, or process disclosed, or represents that its use would not infringe privately owned rights. Reference herein to any specific commercial product, process, or service by trade name, trademark, manufacturer, or otherwise does not necessarily constitute or imply its endorsement, recommendation, or favoring by the United States Government or any agency thereof. The views and opinions of authors expressed herein do not aecessarily state or reflect those of the United States Government or any agency thereof. 


\section{Dedicated to my parents, \\ Harrold James and Hazelyn McComas, \\ for their unwavering support and encouragement.}


List of Figures vii

Vita

Publications $\quad$ ix

Abstract $\quad$ xiii

CHAPTER 1 INTRODUCTION 1

1.0 Magnetotails and Their Field
Reversing Current Sheets

1.1 Intrinsic Magnetotail: Earth 3

1.2 Induced Magnetotails 6

1.2 .1 Venus 7

1.2.2 Comet Giacobini-Zinner 8

$\begin{array}{llll}\text { CHAPTER } 2 & \text { THE NEAR EARTH CROSS TAIL CURRENT SHEET: } \\ & \text { DETAILED ISEE- } 1 \text { AND }-2 \text { CASE STUDIES } & 10\end{array}$

$\begin{array}{ll}2.0 \text { Chapter Overview } & 10\end{array}$

2.1 Introduction 11

2.2 Current Sheet Geometry 14

2.3 Current Sheet Motions 21

2.4 Case Studies: 5 April 197925

2.4.1 15:50 UT 5 April 1979 30

2.4.2 02:00 UT 5 April $1979 \quad 39$

2.4.3 15:15 UT 5 April 1979

2.5 Discussion and Summary 49

2.6 Chapter Acknowledgments 56

CHAPTER 3 THE AVERAGE MAGNETIC FIELD DRAPING AND CONSISTENT PLASMA PROPERTIES OF THE VENUS MAGNETOTAIL 57

3.0 Chapter Overview $\quad 57$

3.1 Introduction 58

3.2 The Average Venus Tail in Magnetic
Coordinates 
TABLE OF CONTENTS (continued)

3.3 The Average Tail Configuration with the Effects of Flapping Removed

3.4 The Inferred Plasma Properties 93

3.5 Summary 103

$\begin{array}{lll}3.6 & \text { Chapter Acknowledgments } & 106\end{array}$

CHAPTER 4 THE GIACOBINI-ZINNER MAGNETOTAIL: TAIL

$\begin{array}{ll} & 108\end{array}$

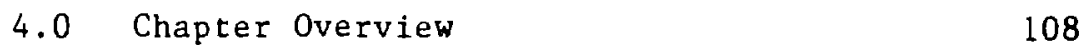

4.1 Introduction 109

4.2 Tail Formation and Topology 113

4.3 MHD Equilibrium 122

4.4 Cometary Mass Transport 145

4.5 Summary and Discussion 153

4.6 Chapter Acknowledgments 162

$\begin{array}{lll}\text { CHAPTER } 5 & \text { SUMMARY AND DISCUSSION } & 164\end{array}$

5.0 Summary 164

5.0 .1 Earth 165

5.0 .2 Venus 167

5.0.3 Comet Giacobini-Zinner 172

5.1 General Discussion $\quad 176$

$\begin{array}{lr}\text { Dissertation Approvals } & 184\end{array}$

$\begin{array}{lr}\text { Acknowledgments } & 185\end{array}$

$\begin{array}{ll}\text { References } & 186\end{array}$ 


\section{LIST OF FIGURES}

Figure

2.1 Schematic of current sheet field lines.

Page

2.2 Schematic of current sheet cross-section.

2.3 Magnetic field and velocity profiles:

15:50 crossing.

2.4 Field data from ISEE-3, IMP-8, and ISEE-2.

2.5 ISEE-2 plasma moments.

2.6 Current density distributions: 15:50 crossing.

2.7 Proton density, temperature, and pressure profiles.

2.8 Three-dimensional velocity profiles:

$15: 50$ crossing.

2.9 Field, velocity, and current density

distributions: 02:00 crossing.

2.10 Field, velocity, and current densicy distributions: $15: 15$ crossing.

3.1 Schematic of Venus magnetotail formation.

3.2 Data set orbital coverage.

3.3 Diamagnetic reduction versus field angle. 69

3.4 Orbit 1761 , diamagnetic reduction. 72

3.5 Field draping angle versus spatial location. 74

3.6 Fraction of tailward pointing lobe. 75

3.7 Lobe field line draping angles. 77

3.8 Schematic of principle underlying constructed coordinate system.

3.9 Magnetic field angle versus $Y^{*}$. $\quad 82$

3.10 Measured $B_{X}$ versus $Y^{*}$.

3.11 Measured $B_{Y *}$ versus $Y^{*}$. $\quad 86$

3.12 Average magnetic field vectors versus $Y^{*}$. $\quad 87$

3.13 Orbital coverage and $B_{Y *}$ versus $X$. 89

3.14 Current density versus $Y^{*}$. $\quad 91$

$3.15 \mathrm{JXB}$ force versus $\mathrm{Y*}$. $\quad 92$

3.16 Derived plasma velocity versus $X$. 97

3.17 Derived plasma acceleration versus $X$. 99 


\section{LIST OF FIGURES (continued)}

Figure

Page

4.1 Schematic of draped magnetic field near comet nuclei.

115

4.2 Flow speed and azimuth.

118

4.3 Schematic of tail/current sheet geometry at time of ICE tail encounter.

119

$\begin{array}{lll}4.4 & \text { Measured plasma and field varlations. } & 123\end{array}$

4.5 Plasma and field pressures.

131

4.6 Inferred ion temperature. 133

4.7 Electromagnetic JxB force. 139

4.8 Derived plasma properties at ICE and upstream near the comet nucleus.

4.9 Schematic of mass flux calculation. $\quad 148$

4.10 Number density and number flux. 150 
May 22, 1958

1980

1980-present

1983-1984

$1984-1985$

1985
Born, Milwaukee, Wisconsin

B.S., Massachusetts Institute of Technology

Staff Member, Los Alamos National Laboratory

Fellowship, Institute for Geophysics and Planetary Physics, Los Alamos Branch

Advanced Study Program Award, Los Alamos National Laboratory

M.S. University of California, Los Angeles

\section{PUBLICATIONS}

1) Radially Uniform Electron Source; D. McComás and S.J. Bame; Review of Scientific Instruments, 53, 1490, 1982.

2) Electron Heating Within the Earth's Bowshock; W.C. Feldman, S.J. Bame, S.P. Gary, J.T. Gosling, D. McComas, M.F. Thomsen, G. Paschmann, N. Sckopke, M.M. Hoppe, C.T. Russe1l; Physical Review Letters, 49, 199, 1982.

3) Electron Velocity Distributions Near the Earch's Bowshock; W.C. Feldman, R.C. Anderson, S.J. Bame, S.P. Gary, J.T. Gosling, D.J. McComas, M.F. Thomsen, G. Paschmann, M.M. Hoppe; Journal of Geophysical Research, 88, 96, 1983.

4) ISPM Solar Wind Plasma Experiment; S.J. Bame, J.P. Glore, D.J. McComas, K.R. Moore, J.C. Chavez, T.J. Ellis, G.R. Peterson, J.H. Temple, F.J. Wymer; ESA Special Publication $\underline{\mathrm{SP}-1050,47,1983 .}$

5) Plasma Regimes in the Deep Geomagnetic Tail: ISEE-3; S.J. Bame, R.C. Anderson, J.R. Asbridge, D.N. Baker, W.C. Feldman, J.T. Gosling, E.W. Hones, Jr., D.J. McComas, R.D. Zwick1; Geophysical Research Letters, 10, 912, 1983. 
6) Channe1 Multiplier Compatible Materials and Lifetime Tests; D.J. McComas and S.J. Bame; Review of Scientific Instruments, 55, 463,1984 .

7) Structure of the Magnetotail at $220 \mathrm{R}_{\mathrm{E}}$ and Its Response to Geomagnetic Activity; E.W. Hones, Jr., D.N. Baker, S.J. Bame, W.C. Feldman, J.T. Gosling, D.J. McComas, R.D. Zwickl, J.A. Slavin, E.J. Smith, B.T. Tsurutani; Geophysical Research Letters, $11,5,1984$.

8) Correlated Dynamical Changes in the Near Earth and Distant Magnetotail Regions: ISEE-3; D.N. Baker, S.J. Bame, W.C. Feldman, J.T. Gosling, P.R. Higbie, E.W. Hones, Jr., D.J. McComas, R.D. Zwickl; Journal of Geophysical Research, 89, 3855,1984 .

9) Evolution of the Earth's Distant Magnetotail: ISEE-3 Eluctron Plasma Results; R.D, Zwickl, D.N. Baker, S.J. Bame, W.C. Feldman, J.T. Gosling, E.W. Hones, Jr., D.J. McComas, B.T. Tsurutani, J.A. Slavin; Juurnal of Geophysical Research, 89, $11007,1984$.

10) Evidence for Slow-Mode Shocks in the Deep Geomagnetic Tail; W.C. Feldman, S.J. Schwartz, S.J. Bame, D.N. Baker, J. Birn, J.T. Gosling, E.W. Hones, Jr., D.J. McComas, J.A. Slavin, E.J. Smith, R.D. Zwick1; Geophysical Research Letters, 11 , 599,1984 .

1i) Plasma Entry into the Distant Tail Lobes: ISEE-3; J.T. Gosling, D.N. Baker, S.J. Bame, E.W. Hones, Jr., D.J. McComas, R.D. Zwickl, J.A. Slavin, E.J. Smith, B.T. Tsurutani; Geophysical Research Letters, $11,1078,1984$.

12) Detailed Examination of a Plasmoid in the Distant Magnetotail with ISEE-3; E.W. Hones, Jr., J. Birn, D.N. Baker, S.J. Bame, W.C. Feldman, D.J. McComas, R.D. Zwick1, J.A. Slavin, E.J. Smith, B.T. Tsurutani; Geophysical Research Letters, 11, 1046 , 1984.

13) The Near Earth Current Sheet: ISEE-1 and -2 Studies; D.J. McComas and C.T. Russe11; Achievements of the International Magnetospheric Study (IMS), ESA Scientific and Technical Publications, SP-217, 205, 1984 .

14) Bistatic LIDAR Expertment Proposed for the Shuttle/Tethered Satellite System Missions; D.J. McConas, H.E. Spence, R.R. Karl, Jr., H.G. Horak, T.D. Wilkerson; Review of Scientific Instruments, 56, 670, 1985 . 
15) The Near Earth Cross Tail Current Sheet: Detailed ISEE-1 and -2 Case Studies; D.J. McComas, C.T. Russell, R.C. Elphic, S.J. Bame; Journal of Geophysical Research, 91, 4287, 1986.

16) The Average Magnetic Field Draping and Consistent Plasma Properties of the Venus Maguetotail; D.J. McComas, H.E. Spence, C.T. Russe11, M.A. Saunders; Journal of Geophysical Research, 91, 7939,1986 .

17) Comet Giacobinni-Zinner: A Plasma Description; S.J. Bame, R.C. Anderson, J.R. Asbridge, D.N. Baker, W.C. Feldman, S.A. Fuselier, J.T. Gosling, D.J, McComas, M.F. Thomsen, D.T. Young, R.D. Zrick1; Science, 233, 356, 1986.

18) The Warped Neutral Sheet and Plasma Sheet in the Near-Earth Geomagnetic Tail; J.T. Gosling, D.J. McComas, M.F. Thomsen, S.J. Bame, C.T. Russell; Journal of Geophysical Research, 91 $7093,1986$.

19) Average Plasma and Magnetic Field Variations in the Distant Magnetotail Associated with Near-Earth Substorm Effects; D.N. Baker, R.C. Anderson, S.J. Bame, R.D. Bellian, P.R. Higbie, D.J. McComas, R.D. Zwickl, J.A. Slavin, E.J. Smith; Submitted to Journal of Geophysical Research, 1985.

20) Plasma and Magnetic Field Variations in the Distant Magnetotail Associated with Near-Earth Substorm Effects; D.N. Baker, S.J. Bame, D.J. McComas, R.D. Zwick1, J.A. Slavin, E.J. Smith; Accepted for publication in Chapman Conference on Magnetotail Physics Monograph, 1986.

21) The Average Configuration of the Induced Venus Magnetotail; D.J. McComas, H.E. Spence, C.T. Russe11; Accepted for publication in Chapman Conference on Magnetotail Physics Monograph, 1986.

22) A Characterization of Large-Amplitude Plasma Fluctuations in the Transition and Sheath Regions of Comet Giacobini-Zinner; D.N. Baker, S.J. Bame, W.C. Feldman, S.P. Gary, D.J. McComas, J. Middleditch, R.D. Zwick1; Geophysical Research Letters, 13, $271,1986$.

23) Three Component Plasma Electron Distributions in the Intermediate Ionized Coma of Comet Giacobini-Zinner; R.D. Zwickl, D.N. Baker, S.J. Bame, W.C. Feldman, S.A. Fuselier, W.F. Huebner, D.J. McComas, D.T. Young; Geophysical Research Letters, 13, $401,1986$. 
24) The Comet/Solar Wind Transition Region at Giacobini-Zinner; M.F. Thomsen, S.J. Bame, W.C. Feldman, J.T. Gosling, D.J. McComas, D.T. Young; Geophysical Research Letters, 13, 393, 1986.

25) Diagnostics of Space Plasmas; S.J. Bame, D.J. McComas, D.T. Young, R.D. Bellian; Accepted by Review of Scientific Instruments, 1986.

26) The Giacobini-Zinner Magnetotail: Tail Configuration and Current Sheet; D.J. McComas, J.T. Gosling, S.J. Bame, J.A. Slavir, E.J. Smith, J.-L. Steinberg; Submitted to Journal of Geophysical Research, 1986. 
FIELD REVERSING MAGNETOTAIL CURRENT SHEETS:

EARTH, VENUS, AND COMET GIACOBINI-ZINNER

by

David John McComas

ABSTRACT

This dissertation examines the field reversing magnetotail current sheets at the earth, Venus, and Comet Glacobini-Zinner. In the near earth study a new analysis technique is developed to calculate the detailed current density distributions within the cross tail current sheet for the first time. This technique removes the effects of a variable sheet velocity by inverting intersatellite timings between the co-orbiting satellites ISEE-1 and -2 . Case studies of three relatively geomagnetically quiet crossings are made; sheet thicknesses and peak current densities are $\sim 1-5 \times 10^{4} \mathrm{~km}$ and $\sim 5-50 \mathrm{nA} / \mathrm{m}^{2}$. Current density distributions reveal a high density central region, lower density shoulders, and considerable fine structure throughout. In the Venus study another new analysis technique is developed to reconstruct the average tail configuration from a correlation between field magnitude and draping angle in a large statistical data set. The variability of the Venus tail data is explained in cerms of 
flapping of the current sheet as the tail tries to maintain a pressure balance condition under varlations in the upstream IMF angle and, therefore, magnetic fluxes in the two lobes. The ayerage magnetic field configuration and consistent plasma properties are derived; the flow velocity varies from $\sim-250 \mathrm{~km} / \mathrm{sec}\left(-8 R_{V}\right)$ to $\sim-470$ $\mathrm{km} / \mathrm{sec}\left(-12 \mathrm{R}_{\mathrm{V}}\right)$, the average sheet and lobe densities are $\sim 0.9$ and $\sim 0.07 \mathrm{amu} / \mathrm{cm}^{3}$, the average $0^{+}$temperature is $-9 \times 10^{7} \mathrm{~K}$, and the integrated tailward mass flux is $\sim 1 \times 10^{26} \mathrm{amu} / \mathrm{sec}$. In the comet study, high resolution magnetic field and plasma electron data from the ICE traversal of Giacobini-Zinner are combined for the first time to determine the tail/current sheet geometry and calculate certain important but unmeasured local ion and upstream properties. Pressure balanco across the tail gives ion temperatures and betas of $\sim 1.2 \times 10^{5} \mathrm{~K}$ and $\sim 40$ in the center of the current sheet to $\sim 1 \times 10^{6} \mathrm{~K}$ and $\sim 3$ in the outer lobes. Axiai stress balance shows that the velocity shear upstrean near the nucleus is $>6$ ( $\sim 1$ at ICE), and that a region of strongly enhanced mass loading (ion source rate $\sim 24$ times that upstream from lobes) exists upstream from the current sheet. The integrated downtail mass $f l u x$ is $\sim 2.6 \times 10^{26} \mathrm{H}_{2} \mathrm{O}^{+} / \mathrm{sec}$, which is only $\sim$ i\% of the independently determined total cometary efflux. 


\section{CHAPTER 1}

Introduction

1.0 Magnetotalis and Their Fleld Reversing Current Sheets

Magnetotails arise out of the solar wind's interaction with magnetic and mass loading planetary and cometary bodies throughout the solar system. While the detalls of magnetotall structures vary from one planetary body to the next, certaln attributes are inherent to magnetotails in general. These include the fuxtaposition of two lobes of nearly oppositely directed magnetic fields and a field reversing current sheet which acts to separate them. Through these current sheets the field rotates from one lobe's orientation to the other, everywhere self-consistently satisfying Ampere's Law.

This dissertation examines three such magnetotalls in general and their fleld reversing current sheets in particular. The three magnetotails are assoclated with very different sorts of inner solar systern bodies. These bodles are: 1) the earth, 2) Venus, and 3) Comet Giacobini-Zinner (G-Z). These three were chosen not just because of their considerable scientific interest, but also because each represented a different type of interaction with the solar wind. By examining the different manifestations of magnetotalis and fleld reversing current sheets, this dissertation attempts to convey some of the richness of variety which these structures display.

Time dependent effects are often extremely important in 
determining the instantaneous structure and properties of magnetotalls and their current sheets. Examples of such effects include variations of the solar wind and Interplanetary Magnetic Field (IMF) properties upstream from all three bodles, near earth reconnection and consequent substorm activity at earth, variations In the near nightside magnetic structure at Venus, and spatial and temporal fluctuations in the neutral outgassing rate of Comet $G-Z$. While these effects are all extremely interesting in their own rights, the fundamental, average magnetotall/current sheet properties are time independent. In order, therefore, to examine the fundamental current sheet properties within the three magnetotalls, this dissertation concentrates on the time independent aspects.

The funcamental planetary/cometary property which dominates a body"s interaction with the solar wind and, therefore, the nature of the magnetota11 which forms antisunward from $1 \mathrm{t}$, is the magnetohydrodynamic (MHD) obstacle which it presents to the flowing solar wind/IMF. At bodles with substantial intrinsic magnetic fields, such as the earth, Mercury, Juplter, Saturn, and Uranus, the solar wind flow is essentially diverted entirely by the region dominated by this intrinsic field. At bodies which lack substantial intrinsic magnetic flelds but possess appreclable gaseous atmospheres, such as ccmets, Venus, and some of the outer solar system planetary sateliftes, mass loading of the flow and the formation of a conductive lonosphere serve to divert the solar wind flow. For these atmospheric obstacles, the presence (Venus) or absence (Comet 
G-Z) of a substantial gravitational field determines whether a compact or extended mass loading region is formed.

The flowing solar wind/IMF can be diverted about a body by elther an intrinsic planetary magnetic field or by mass loading of the flow and the formation of a conductive lorosphere. of course some bodies in the solar system have neither an intrinsic magnetic field nor a sufficient atmosphere to mass load the solar wind or produce a conductive ionosphere. The moon and, probably, asteroids also, are examples of such bodies. The region antisunward of such an obstacle, however, is not a magnetotail, but rather a wake. These wakes are essentially devold of plasma since the solar wind is not deflected about the obstacle, but is absorbed as it collides with the body's surface. Since magnetotalls and magnetotail current sheets are not formed at such obstacles, they will not be considered further in this dissertation.

1.1 Intrinsic Magnetotail: Earth

of the three magnetotalis studied in this dissertation, oniy the earth's contalns magnetic fields which are connected to the planetary obstacle. When a planetary body possesses a substantial Intrinsic magnetic fleld, as does the earth, the flowing solar wind acts to deform the Intrinsic magnetic cavity surrounding the planet. This has the effect of compressing the cavity on the dayside and stretching it out on the nightside into a tall-like configuration. Tallward elongation of the magnetosphere occurs both because of tangential stresses or drag at the magnetopause boundary and because 
of magnetic reconnection between the planet's intrinsic magnetic field and the IMF.

Magnetic reconnection between the shocked IMF (magnetosheath field) and the Intrinsic planetary fleld occurs preferentially near the nose of the magnetopause at times when the two fields are oppositely directed (1.e., when the IMF has a negative $\mathrm{B}_{\mathrm{Z}}$-comporont). Interconnection between the magnetosheath and lobe flelds travels tallward along the magnetopause, and the magnetosheath flow thereby acts to drape back the magnetic fleld in the tall into nearly opposttely directed lobes. In contrast to the lobes, where the magnetic fleld at least partially interconnects the planetary Ionosphere and the IMF, the near earth cross tall current sheet consists of field lines which are connected to the lonosphere at both ends. In the steady state picture, the near earth current sheet plasma and magnetic fleld are slowly convected earthward from a region where the two lobes are undergoing steady state reconnecting, somewhere deep in the tat $t$.

In light of the already very substantial knowledge of the general characteristics of the terrestrial magnetotail and its field reversing current sheet, this dissertation avolds a reexamination of these general properties. Rather, it extends the frontier of knowledge by determining detalled current density distributions in the near earth cross tall current sheet for the first time. This study is carried out in Chapter 2 through the development and use of a new multisatelite data analysis technique.

High resolution magnetic fleld and plasma data from the 
co-orbiting satelites ISEE-1 and -2 are combined in order to resolve the current sheet geometry and orfentation at the time of spacecraft crossings. The magnetic fleld data is then used to make careful intersatellite timings of the motion of the current sheet and derive the detailed cross tail current density distributions through the sheet. These distributions will be shown to exhibit substantial fine structure, and the 1mpilcations of this structure w111 be discussed.

The orlentation of the orb1ts of the ISEE-1 and -2 spacecraft remain essentially fixed throughout the year so that they are in an advantageous position to sound the near magnetotall for only approximately four months each year (mid-February to mid-June). During the years since the 1977 launch, the orbits have slowly evolved so that the spacesraft spend progressively more time in the southern lobe and make fewer current sheet crossings. Consequently, the special criterla required to resoive the current sheet geometry w1th only two spacecraft, as described in detail in Chapter 2, are far less likely to occur later in the mission.

In any case, the magnetic fleld data for the four month Intervals have been carefully examined from the start of mission through 1983. From the few analyzable crossings found in the data, three whlch were almost certainly not assoclated with substorm activity were chosen for detalled analysis. In this way, the average or steady state structure of the current sheet is most nearly examined. In the future, the European Space Agency CLUSTER Mission, which is designed to combine four co-orbiting satelites, 
should be able to make substantial use of the new technique developed in this section.

\subsection{Induced Magnetotalls}

At planetary and cometary bodies which possess substantial atmospheres but no intrinsic magnetic fields, magnetotails can st111 form from the solar wind Interaction. Unlike the case of intrinsic magnetotalls such as the earth's, however, the magnetic fields comprising induced magnetotalls are of purely solar origin. The plasma in induced magnetotalls, on the other hand, is largely of planetary or cometary origin and has been added to the flow through photolonization, charge exchange, or other similar mass loading processes.

The general solar wind/IMF interaction with such bodies consists of draping of the magnetic field due to mass addition and slowing of the flow, and is described in more detall in the introductory sections of Chapters 3 and 4. Briefly, the magnetic field Immediately adjacent to the tall continues to be carried along by the relatively unimpeded flow while the flow passing near to the obstacle is mass loaded and slowed. In addition, formation of a conductive lonosphere serves to exclude the upstream flow, further slowing and diverting it. Since the fleld links the regions of greater and lesser flow speeds, the fleld configuration becomes highly draped and a magnetotall configuration forms, complete with oppositely directed tall lobes and a self-consistent, field reversing current sheet. 


\section{2 .1 Venus}

Venus represents an example of an induced magnetotall forming body which has an essentially gravitationally bound newtral atmosphere. As such, the amount of neutral gas above the Venus Lonosphere is $11 \mathrm{~m} 1 \mathrm{ted}$ and drops off very quickly with distance from the planet. The plasma flow directly upstream from the planet is therefore slowed over a relatively short distance, and the fleld directly upstream piles up above the dayside tonopause. The magnetotall which forms in this interaction is demarked by relatively sharp and wel1-defined tall boundarles.

Even though the Pioneer Venus Orbiter, PVO, has been in orbit around Venus since 1978, the detalled average properties of the Venus magnetotal1 have not been determined prior to the work described in Chapter 3 of this dissertation. This was due to the extremely large amount of varlability of the tal1's magnetic configuration and location, due to fiuctuations in the upstream IMF orlentation and solis wind properties, which occurs on a few-minute time scale. Exacerbating this problem is the general lack of plasma observations attainable within the tall with any of the plasma analyzers carrled onbcard PVO. Therefore, unlike the earth study in Chapter 2, the Venus magnetotall study described in Chapter 3 centers on the more general, average properties of the Venus magnetota1\% and its field reversing current sheet.

In order to carry out such a study, which would provide much wore Information about the average configuration and properties than previous case studies of the tall, a new method for analyzing the 
highly variable tall field data was needed. In the Venus chapter of this dissertation fust such a technique is developed. This technique allows the data to be organized by distance from the fleld reversing current sheet rather than by location in Inertial space. Nearly 10,000 one-minute averaged magnetic fleld data samples are statistically examined in these new coordinates, and the average magnetic configuration of the Venus inagnetotall is determined. From this derived average fleld configuration, and using an MHD analysis, the self-consistent, average plasma properties of the tall are derived for the first time.

\subsubsection{Comet Giacobini-Zinner}

While many aspects of cometary interactions with the solar wind are similar to those at Venus, the extremely small gravitational attraction of a comet combined with its continual gas efflux causes the cometary obstacle to be much more extended. Inside the extended region of mass loading, a cometary lonopause is expected to form near to the nucleus where the Ion density is so large that at least partial exclusion of the upstream field occurs. Ion talls observed visually from earth and inftial G-Z magnetotall results indicate that cometary magnetotalls apparently also have well-defined boundaries, even though the global cometary interactions are so extended.

The magnetotall traversal of Comet Glacobini-zinner by the International Cometary Explorer (ICE) on 11 September 1985 represents the first, and only, scheduled, in situ observations of a 
cometary magnetotall. Unlike the Venus tall studied in Chapter 3, the G-2 magnetotall data set consists of only a single tall crossing. Therefore, no statistical derlvation of the average tail properties is possible, and the observed properties of the tall must simply be assumed to be typical. Also unlike the Venus case, however, the plasma electron properties of the crossing were measured along with the magnetic fleld properties, allowing for a much better analysis of the single crossing data sets.

In Chapter 4 of this dissertation the high resolution magnetic fleld and plasma data sets for the ICE tall traversal are combined for the first time. Together they allow for the determination of the magnetotall configuration at the time of the spacecraft crossing. From an MHD analysis of these combined daca, the upstream conditions at the average plck-up locations near to the comet nucleus are also derived, and with tliem the detalled tall formation process is examined. 
The Near Earth Cross Tall Current Sheet:

Detalled ISEE-1 and -2 Case Studies

\subsection{Chapter Overview}

Three near geomagnetic tall current sheet crossings of the ISEE- $i$ and -2 satellites on 5 April 1979 are examined in detafl. All are associated with the passagge of an 1nterplanetary shock and the region of varlable solar wind pressure behind 1t. The general geometry of fleld reversing current sheets is discussed, and this geometry is examined for the cases studied, by using the ISEE-1 and -2 co-orbiting satellite data sets. A new technique is employed which removes the effects of a variable sheet normal velocity for the first time. This allows us to calculate firm upper bounds on the current sheet thicknesses, and by utilizing certain physicaliy motivated assumptions, detirmine the most probable actual sheet thicknesses, and inclinations of the fleld lines within these sheets. Current density profiles derived with this technique show the main cross tafl current sheet to be a structure that is many thermal ion-gyroradif thick and which is sometimes embedded in a region that is three or more times thicker and contains much smaller current densities. These profiles also exhibit a considerable amount of fine structure in the sheet which appears as narrow peaks in the current density distributions. Possible explanations for 
these structures, and for the overall sheet structure itself, are examined.

\subsection{Introduction}

Current sheets are ublquitous in space plasmas. These sheets carry current distributions through regions of spatially varying magnetic flelds such that the current density and magnetic field configurations everywhere self-consistently satisfy Ampere's Law. One interesting class of current sheets, fleld reversing sheets, forms when two nearly antiparallel portions of a magnetic fleld abut each other. Interplanetary sector boundaries and lobe separating magnetotall current sheets are examples of fleld reversing sheets. Since this study will examine the near earth, cross tall current sheet which serves to separate the oppositely directed magnetic fleids in the geomagnetic tall lobes, a brief discussion of such sheets in intrinsic magnetotalls is appropriate.

Intrinsic magnetotall current sheets form in the central portions of magnetotails antisunward of planets with intrinsic magnetic flelda, such as the earth, Jupiter, Saturn, and Mercury. The solar wind deforms the intrinsic planetary field, stretching out field lines benind the planet. In addition, dayside merging causes magnetic flux to be added to the tall lobes. A magnetotall configuration $1 \mathrm{~s}$ therefore formed, with adjacent lobes of roughly antiparallel fields separated by a central cross tall current sheet.

In the theoretical $11 \mathrm{mlt}$ of the so-called Harris neutral sheet [Harris, 1962], a one-dimensional equilibrium current sheet solution 
Is found for a current sheet separating antiparallel fields. In actual planetary magnetotalls, reconnection and three-dimensional effects can alter this geometry and create regions of very different magnetic topologies. Planetward of an X-type neutral 11ne, fleld Iines connect to the ionosphere. Stresses in the magnetotall are communicated to the lonosphere, and the Lonospheric responses are in turn communicated to the tall. Tallward of an $X-1$ ine on reconnected interplanetary magnetic field (IMF) lines, the boundary conditions are vastly different. Current sheets in these disconnected portions of the tal1 have magnetic topologies similar to Induced magnetotall current sheets such as are observed at Venus [Russe11 et al., 1981] and postulated to exist antisunward of comets. Such a field geometry is highly kinked and mass loaded throughout the sheet. The magnetic field tension acts to accelerate the plasma and reduce the total sheet current by restraightening the field to the local IMF configuration. Another type of magnetic field topology can form when reconnection inftiates on closed (i.e., connected to the farth at both ends) fleld 11nes, namely an 0-type neutial line [Russel1, 1974] or plasmoid [Hones, 1979]. The fully three-dimensional magnetic configurations of these structures and their associated current systems are quite complicated, and are an area of considerable current research [Hones et a1., 1982, 1984]. This study is concerned with the simple, field reversing, near earth current sheet, and will not examine disconnected or plasmotd-embedded sheets.

Prior to the launch of the co-orbiting satellites, ISEE-1 and 
-2 , studles of current and plasma sheet topologles and dynamics had necessarlly relied on data taken from single or widely separated spacecraft. The use of single spacecraft data sets is useful for the purpose of maklng large statistical studies of average current sheet properties (e.g., Fairfield [1979], [1980]), but it 1s Impossible to separate spatial and temporal effects with data taken from only one location. Even with two-spacecraft observations a fully three-dimensional geometry which may vary as a function of time cannot be uniquely resolved. Magnetic fleld topologies in current sheets may, in general, be quite complicated (see, for example, Lui [1983]), and the sheet may even have closed magnetic structures embedded in it. For the purposes of this study, however, only current sheet crossings which exhibit planar, monotonic field variations have been chosen. Th1s greatly simplifies the problem of resolving the sheet geometry with two-spacecraft observations. In addition to these constraints, the intersatellite separation vector must 11 e well out of the plane of the curzent sheet in order to make a reliable determination with our technique. Out of all of the ISEE-1 and -2 current sheet crossings from the 1978 and 1979 ta11 orbital seasons, six crossings were identified which exhibited fast and relatively smooth, monotonic fleld varlations in the data sets of both of the spacecraft, and for which the intersatellite separation vectors are a sultably large angle from the planes of the current sheets.

It is the purpose of this paper to discuss the geomagnetic cross tall current sheet in the near earth region through the 
detalled examination of three case studies. Very fast sheet crossings were chosen in order to minimize the effect of temporal varlations of the sheet structure 1tself. Since the geomagnetic tall, In general, and the plasma sheet, In particular, are subject to large and rapid vartations as a function of substorm phase, [McPherron et al., 1973; Hones, 1979, and references tíerein] current sheet crossings were specifically chosen to avold times of large substorm activity. Speciflcally, all three were chosen from a day when the variable pressure region behind an interplanetary shock caused large scale motions of the magnetotall and repeated encounters between the current sheet and the ISEE-1 and -2 satellices. Bulk reorlentations of the magnetotail, due to varlations in the solar wind flow, caused substantiaily enhanced geomagnetic activity on this day. Therefore, the crossings studied here cannot be considered to be representative of "quiet" current sheet crossings. They do, however, probably represent crossings caused by bulk motions of the tail, and not internal: substorm related reconfigurations, as will be discussed in greater detail in following sections.

\subsection{Current Sheet Geometry}

The natural coordinate system for examining simple sheet crossings is a local, current sheet normal coordinate system. In this system, the current sheet is assumed to have surface boundarles which are planar and parallel on the size scale of the satellite separation vector. Magnetic fleld lines rotate through the current 
sheet so that the flelds on opposite sides of the sheet are roughly antiparallel. Since only simple sheet crossings are examined here, the fleld rotation through the sheet is assumed to be symmetric about the current sheet midplane. Th1s quite reasonable assumption greatly reduces the ambigulty in the fleld geometry since the axis of symmetry is easily resolved from single satellite magnetometer data alone. This axis is defined to be the direction in which the field undergoes its maximum variance for the crossing, and is labeled as the L-axis throughout this study. The ratio of the maximum to the intermediate eigen values from the minimum variance analysis indicates how well this axis is defined.

Figure 2.1 shows the fleld geometry of a simple current sheet In the boundary normal coordinate systen. The $\mathrm{N}$-axis defines the current sheet normal, while the M-axis lies in the plane of the sheet and completes the right-handed set. In the nominally expected magnetotall configuration, the $L, M, N$ axes would correspond roughly to the GSM $X, Y, Z$ coordinates, respectively. The $L, M, N$ coordinates, however, describe a local coordinate system which may be quite different from the nominal configuration if the sheet surface is wavy, as has been suggested of the plasma sheet boundary by I:1 et a1. [1978], or if the tall becomes torqued around by the IMF ${ }^{B}{ }_{Y}$-component, as is suggested to occur in the deep geomagnetic tall [S1beck et a1., 1985].

For the current sheet crossings examined in this study the magnetic field rotations are essentially confined to a plane, which 1s defined to be the $L$, $n$ plane for the purposes of this study. In 


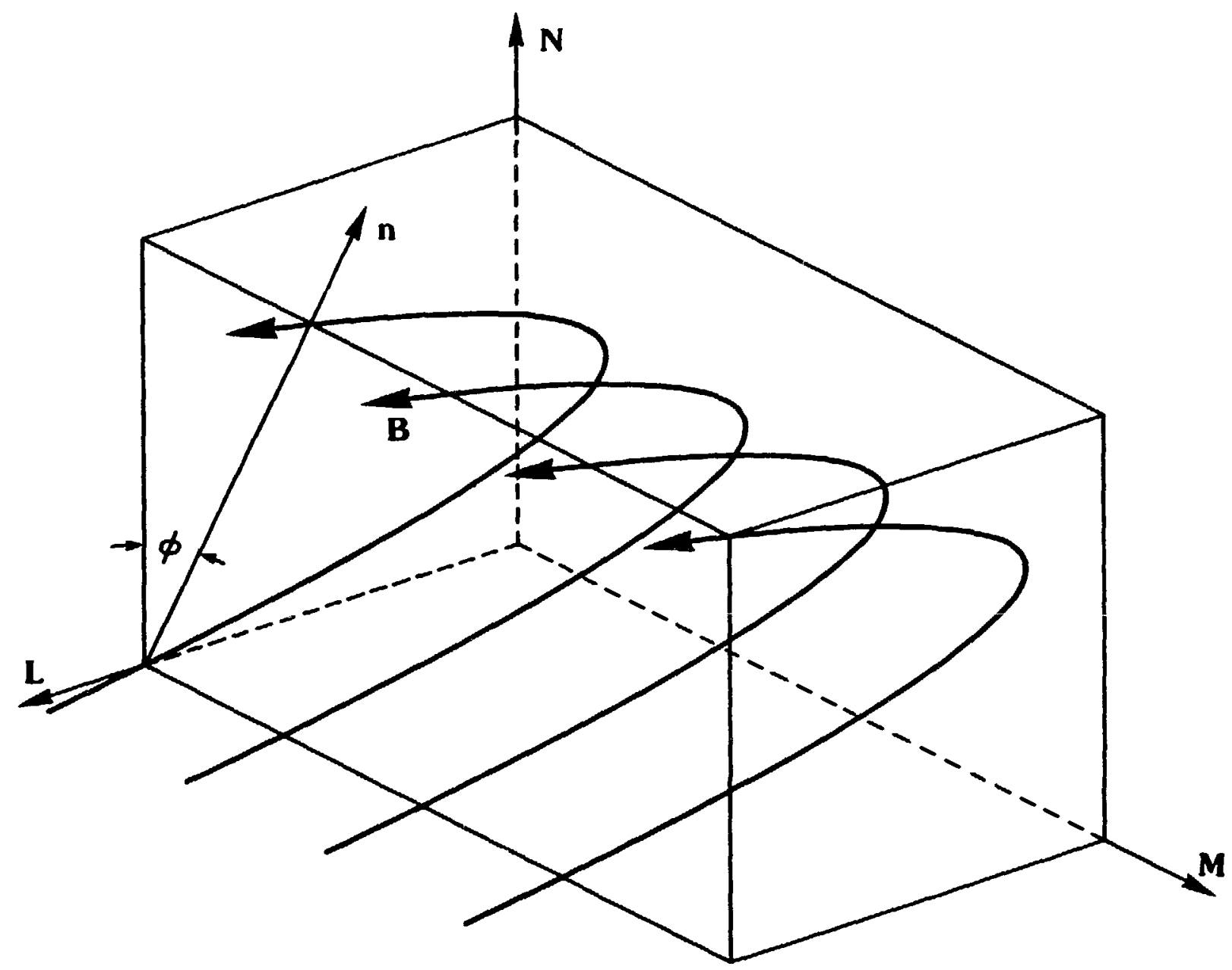

Fig. 2.1. Magnetic field line rotations in the cross tail current sheet. The planes of the field lines are inclined with respect to the sheet normal by the angle $\phi$. The $L$ axis bisects the field rotation while current flows self-consistently in the $M$ direction. 
practice, the $m$ - and $n$-axes are chosen so that the magnitude of $B_{m}$ and $B_{n}$ are essentially zero and constant respectively, through the crossing. of course it is possible that the magnetic field rotation through the sheet is not well confined to a plane, making it Impossible to find an axis such as $m$ for which the field component remains essentially zero through the crossing. If the sheet crossing does not have the simple symmetric and planar properties delineated above, then the structure of the current sheet at that time and location must be more complicated than the simple model, and the case is refected. In general, however, this criterion has been satisfled by the simple crossings which were chosen from overview data plots. The plane defined by the magnetic fleld rotation, as shown in Figure 2.1 , may in general be inclined by the arbitrary angle, $\phi$, with respect to the current sheet normal, N.

Once the geometry has been fully determined, spatial and temporal effects can be separated in the dual satellite data by careful intersatelife timing. For a current sheet with planar, parallel surfaces, as assumed here, only the component of the sheet motion perpendicular to the surface (In the N-direction) is observable with intersatellite timing. Unfortunately, the current sheet geometry is not fully determined as is demonstrated in Figure 2.2. In this figure, the geometry has been reduced to the plane, $\mathrm{P}$, of the four coplanar vectors; $M, N, m$, and $n$. This is possible because the L-axis $1 \mathrm{~s}$ uniquely known, and motions of the sheet parallel to the L-axis are unobservable with intersatellite timing, since the sheet is assumed to be uniform in the L-direction. 


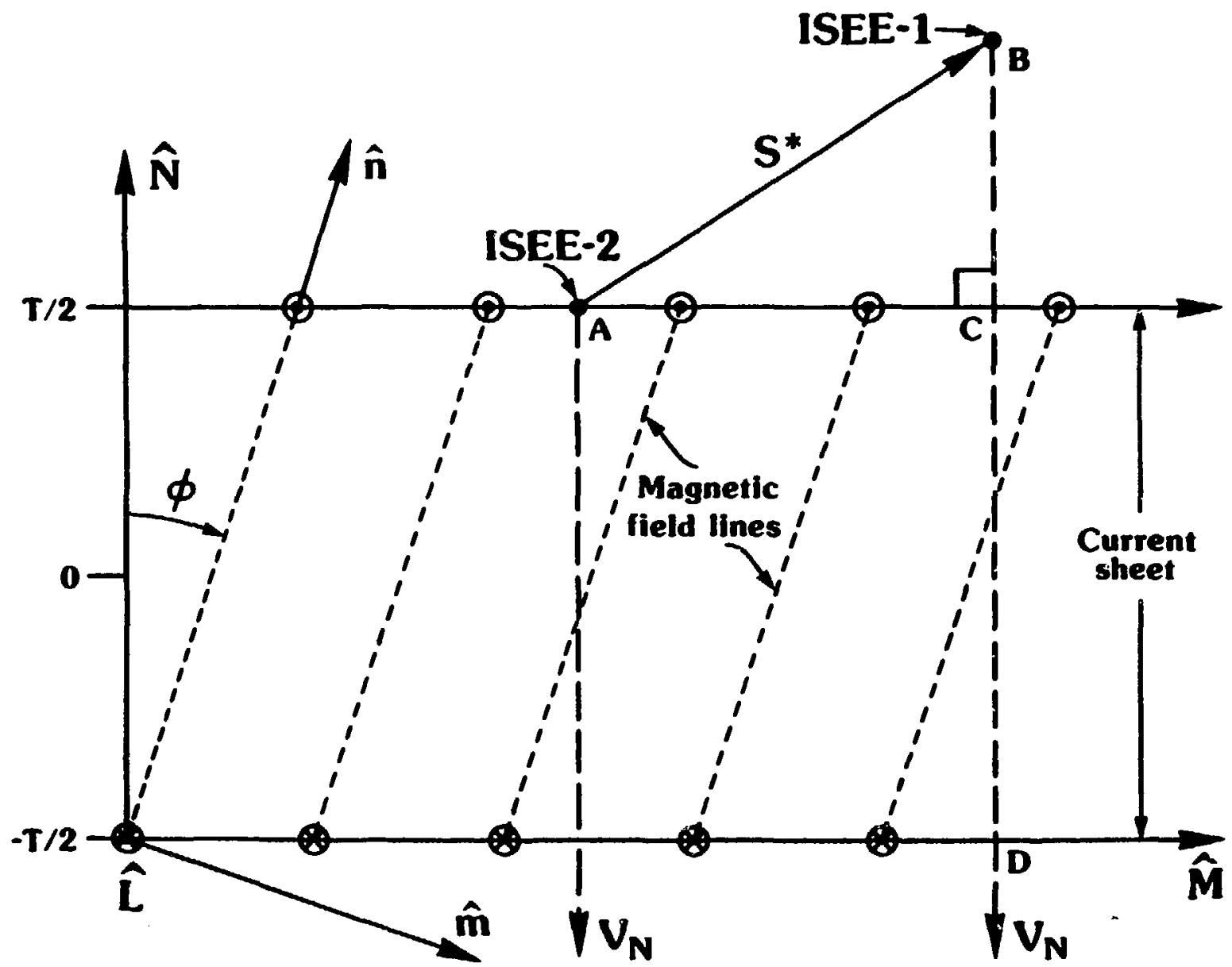

Fig. 2.2. Cross sectional cut through the current sheet in the plane perpendicular to the $L$ axis. $S^{*}$ is the projection of the satellite separation vector into this plane. The field 1ine axes, m and $n$, are well defined as is $S^{*}$, but the angle to the actual sheet normal $\phi$, is not known. 
The $\mathrm{m}$ - and $\mathrm{n}$-axes are also well known, as described previously, but the arbitrary rotation angle, $\phi$, is completely unknown. This ambiguity in the geometry is a central theme for this study. By making physically motivated assumptions about the geometry, the structure of the sheet is examined and this ambiguity is partially resolved.

Since the normal to plane $P$, namely $L$, is well specified, it is possible to obtain the component of any known vector, $A$, in the

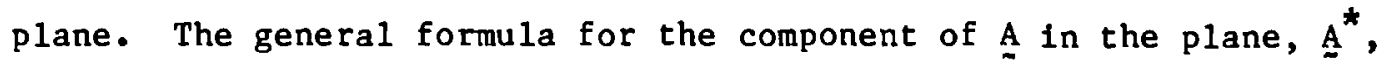
is $\underline{A}^{*}=\hat{L} \times(\underline{A} \times \hat{L})$. This method is used, for example, to determine the component of the $\mathrm{Z}_{\mathrm{GSM}}$-axis in this plane. The unft vector in this direction in the plane is called $\hat{\mathrm{N}}_{\mathrm{NOM}}$ as it represents the expected nominal direction for the sheet normal given that the $\mathrm{X}_{\mathrm{GSM}}$ axis has been rotated to the L-direction. In particular, $\hat{\mathrm{N}}_{N O M} \propto \hat{\mathrm{L}} \times\left(\hat{\mathrm{Z}}_{\mathrm{GSM}} \times \hat{\mathrm{L}}\right)$, and is a well speclfied vector since both the L-and $\mathrm{Z}_{G S M}-$ directions are well specified and usually nearly orthogonal. This definition of the nominal normal will be used in the ensulng sections to provide a baseline current sheet geometry consistent with the observed field rotations.

The component of the ISEE I-2 separation vector which lies in the plane $P$, is labeled $S^{*}$ in Figure 2.2. $S^{*}$ is similarly defined by $\underline{S}^{*}=\hat{\mathrm{L}} \times(\underline{S} \times \hat{\mathrm{L}})$, where the separation vector, $\underset{\sim}{S}$, and the maximum varlance axis, $L$, are both well specified. Just as for the Ldirection, the component of $S^{*}$ parallel to the M-axis is also unobservable with the intersatellite timing since the sheet is assumed uniform in the M-direction. Only the component of the 
separation vector parallel to the $\mathrm{N}$-axis, $S_{N}=\underline{S} \cdot \hat{N}$ is used in determining the sheet normal velocity.

If a constant normal velocity, $v_{N}$, is assumed, then this velocity can be determined by dividing $S_{N}$ by the time lag, $\Delta \tau_{B C}$, between the initial encounters with the sheet seen at the two spacecraft (points $B$ and $C$ in Figure 2.2). The thickness of the sheet, $T$, is then simply the product of the normal velocity, $v_{N}$, and the duration of the crossing, $\Delta \tau_{\mathrm{CD}}$. This type of analysis has been discussed in greater detall by McComas and Russell [1984] . Figure 2.2 is drawn with the satellites moving in the $\mathrm{N}$-direction which is geometrically equivalent to the actual case of the sheet moving in the minus $\mathrm{N}$-direction on the short time scale of the crossing. It will be shown in the next section that a varlable velocity profile can also be approximately calculated from a sertes of simflar timings and, therefore, the effect of this variation can be essentially removed from the data.

It is possible to calculate a maximum current sheet thickness for any crossing by finding the sheet thickness parallel to the arbitrarily oriented vector $\mathrm{s}^{*}$. This is accomplished by letting $s_{N}=\left|S^{*}\right|$, since $s_{N}=s \cdot \hat{N}<\left|S^{*}\right|$. This 1imiting thickness, TMAX, would be highly variable, even if all current sheets were the same thickness, since the angles between $\underline{S}^{*}$ and $\hat{\mathrm{N}}$ are arbitrary. These thicknesses in all cases, however, set firm upper bounds on the actual sheet thicknesses. 


\subsection{Current Sheet Motions}

It is critically important to determine the current sheet normal velocity in order to measure the basic sheet properties such as thickness and current density distribution. While an average crossing velocity is sufficlent to determine the sheet thickness and total integrated current, a detalled knowledge of the Instantaneous velocity as a function of time, through the crossing, is required to calculate the exact current density distribution. This is because the current density is calculated from the curl of the magnetic field by Ampere's Law, $\nabla \times \underline{B}=\mu_{0} J$. For the typical field geometry found in the near magnetotall $B_{L}$ is not a strong function of $M$, and both $B_{M}$ and $B_{N}$ have roughly constant, non-zero values. Since they are comparatively weak functions of the spatial coordinates, they do not contribute substantially to the curl of $\underset{\sim}{ }$, and currents are driven essentially parallel to the M-axis. The curl of the field in the $L, M, N$ coordinate system is therefore simply $\mu_{0} J_{M}=\partial B_{L} / \partial N$, where $\partial B_{L}$ and $\partial N$ are replaced in practice by $\Delta B_{L}$ and $\Delta N=V_{N} \Delta t$, respectively. If $v_{N}$ is an unknown function of time, the current density distribution is indeterminate. This essential ambigulty has made it impossible to calculate rellable current density distributions in current sheets unt11 now.

We have developed a program to calculate the sheet normal velocity as a function of time. The only assumption required for this analysis is that all locations within the sheet and in the region of the crossing which have equal $B_{L}$ magnitudes occur at the same distance from the sheet midplane. This assumption, however, is 
not new since it is equivalent to assuming that the sheet is constant in $L$ and $M$ on the size scale of the satelitte separation vector, and does not change much on the intersatelifte timing timescales. Since the separation vector is typically only a few thousand kllometers, and since the crossings are selected specifically to be of short duration, typlcally a few minutes, this assumption is reasonably valld at all but active substorm times.

Another effect which will tend to complicate our analysis is the possibility of compressional waves in the plasma sheet. Standing compressional waves, however, should produce quasi-periodic variations of the field which are not observed in the crossings studied here. The effect of impulsive (non-periodic) compressional waves is to reduce the accuracy of our technique by superimposing "noise" on the larger varlations of the current sheet crossings. These waves, however, w111 tend to cause $B_{L}$ to vary non-monotonically across the sheet and, since we only analyze crossings with monotonic $B_{L}$ variations in this study, such waves cannot be of primary importance for cases studied here.

The magnetic signature of a typical quick current sheet crossing, which occurred between 15:42 and 15:56 UT on 5 Apr11 1979, is shown in the upper panel of Figure 2.3. The data are shown in the $L, m, n$ coordinate system, and a small data gap has been filled in the $\mathrm{B}_{\mathrm{L}}$-component by interpolation, just after 15:53. The magnitudes of $B_{L 2}$ at $t_{1}$ and $B_{L 1}$ at $t_{j}$ are equal by construction, as are the magnitudes of ${ }^{B_{L 2}}$ at $t_{j}$ and $B_{L 1}$ at $t_{k} \cdot$ The time lags between the two points, $t_{1 a g}$, are simply $t_{j}-t_{1}$, and $t_{k}-t_{j}$, for the two 


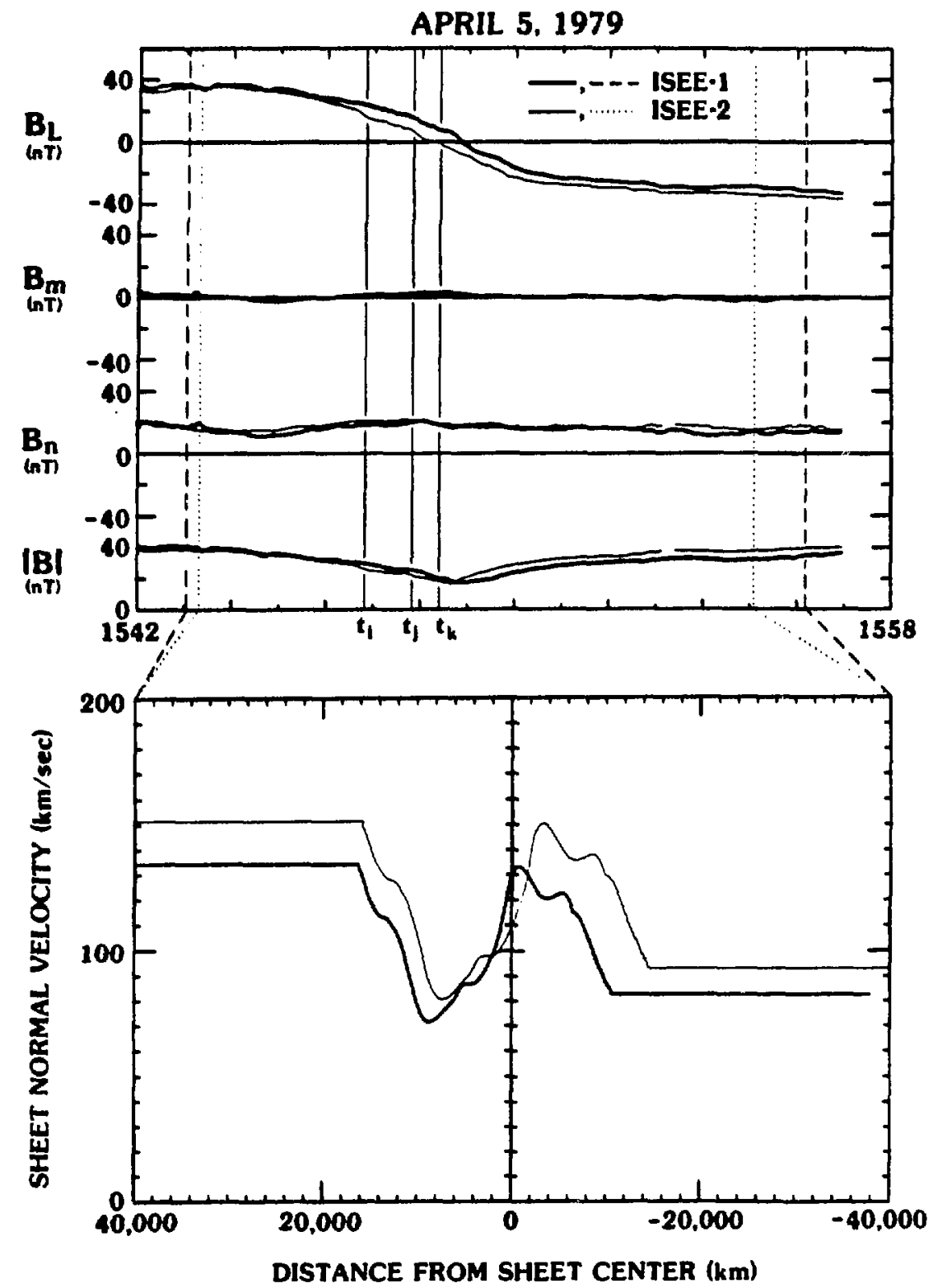

Fig. 2.3. Magnetic field data in the $L, m, n$ coordinate system for the crossing which occurred at $\sim 15: 50$ UT at $-17.6,-3.2,0.2 R_{G} G S M$ (top pane1). Intersatellite timing is derived for each distance from the current sheet center, and these timings are inverted to yield the sheet normal velocity as a function of location (see text for details). Note that $B_{m} * 0$ while $B_{*} \approx$ constant value, as expected for a field line rotating in the $L, n$ plane. The bottom panel displays the calculated velocities as a function of location in the sheet. The horizontal portions on the ends are simply extrapolations of the last reliably determined velocitles. 
perlods, respectively. By simllarly calculating $t_{1 a g}$ at all times through the crossing, an approximately normal crossing velocity as a function of time can be deduced.

The sheet normal velocity calculated for any particular tag, bounded by the times when the first satelifte reaches a particular ${ }^{B} \mathrm{~L}$ value and when the second satelitte reaches the same $B_{L}$ value, $1 \mathrm{~s}$ an average velocity over all Instantaneous velocitles between these two times. St111 referring to the upper panel of Figure 2.3 , the actual instantaneous velocity at time $t_{j}$ is averaged into all velocities calculated from the time lags which begin or end at any time between $t_{1}$ and $t_{k}$. For a velocity profile which is relatively constant on the timescale of $t_{1 a g}$, the approximate velocity at time $t_{j}$ is just the average of the velocities calculated using the time 1 ag method between times $t_{1}$ and $t_{k}$. With this method an approximate normal velocity crossing profile is dertved for the entire central portion of the sheet crossing. This method breaks down only on the sides of the crossing since the two curves converge there and intersatelite timing becomes Impossible. The fact that the two do not completely converge on the right-hand side of the crossing shown Indicates that the assumption of a planar, parallel geometry is not strictly true, or that the satellites did not sound completely through on this side of the sheet crossing.

The lower panel of Figure 2.3 shows the normal velocity profiles for the ISEE-1 and -2 spacecraft for the crossing. Prlor to calculating this velocity profile, the field data in the upper panel was filtered with a Kalser low pass filter with a cutoff 
frequency of $.025 \mathrm{~Hz}$. This value was chosen since it corresponds to a timescale of 40 seconds, which is a typical value for the time lags between the two spacecraft, determined as described previously for this crossing. Filtering the fleld data with a cutoff value of $t_{1 a g}$ insures that a reliable velocity profile is derived, and this procedure is carried out for all cases in this study. The constant velocity sections at the two ends are simply extensions of the last rellable velocity calculated.

The procedure described here, and the program which has been developed to Implement 1t, provides a number of important advantages over simply assuming a constant normal velocity, as has been done previously. The technique, however, does not derive the actual Instantaneous velocity profile, but rather a smoothed version of this profile where high frequency varlations $\left(\tau<t_{\text {lag }}\right)$ have been filtered out. This technique therefore provides the best results when $t_{\text {lag }}$ is typlcally much smaller than the total $t$ ime of the crossing. Higher time resolution velocity profiles are undetermined from the two-satelite data available. The averaging technique described herein provides the best solution for the sheet normal velocity with the only caveat that those structures seen which correspond to timescales less than tigg, are not reliably determined.

2.4. Case Stud1es: 5 Apr11 1979

Just prior to 2:00 UT on 5 Apri1 1979, an 1nterplanetary shock arrived at IMP-8 which was located in the solar wind in near earth 
orbit at approximately $(-2,22,27) R_{E}$ In GSM coordinates. The IMF magnitude at this location rose from a value of $13 \mathrm{nT}$ upstream of the shock to $\sim 60 \mathrm{nT}$ just downstream, and persisted unt11 15:00 UT when 1 t began to drop back to the pre-shock value which it reached at about 21:00 UT. The top two panels of Figure 2.4 show the ent1re day of magnetometer data taken $220 R_{E}$ upstream at ISEE-3, and taken near the magnetosphere at IMP-8, respectively. The traces are quite similar, although shifted in time approximately forty minutes, which 1 s consistent with the solar wind tcansit time for $200 \mathrm{R}_{E}$. These observations suggest that the shock and related structures have spatial scale sizes sufflclently large to affect the magnetosphere as a whole. The downstream region is marked by numerous field rotations and variations in the total field strength. ISEE-3 plasma data (not shown) indicate that the number density and flow velocity are highly variable throughout the post-shock region. The plasma pressure fumps across the shock by about a factor of seven, and is also highly variable $\left(P_{\max } / P_{m 1 n} \sim 30\right)$ throughout the day. These variations in flow direction and solar wind pressure cause substantial variations in the compression and orientacton of the geomagietic tall. On this day the co-orbiting satellftes, ISEE-1 and -2 , were traversing near the midplane of the geotali and, therefore, were in an 1deal position to cross the tail current sheet. The magnetometer data from ISEE-2 for this entire day is shown In the bottom panel of Figure 2.4. Reversals of the $x$ component of the field show that the current sheet was repeatedly encountered and crossed. From this set of partial and complete 


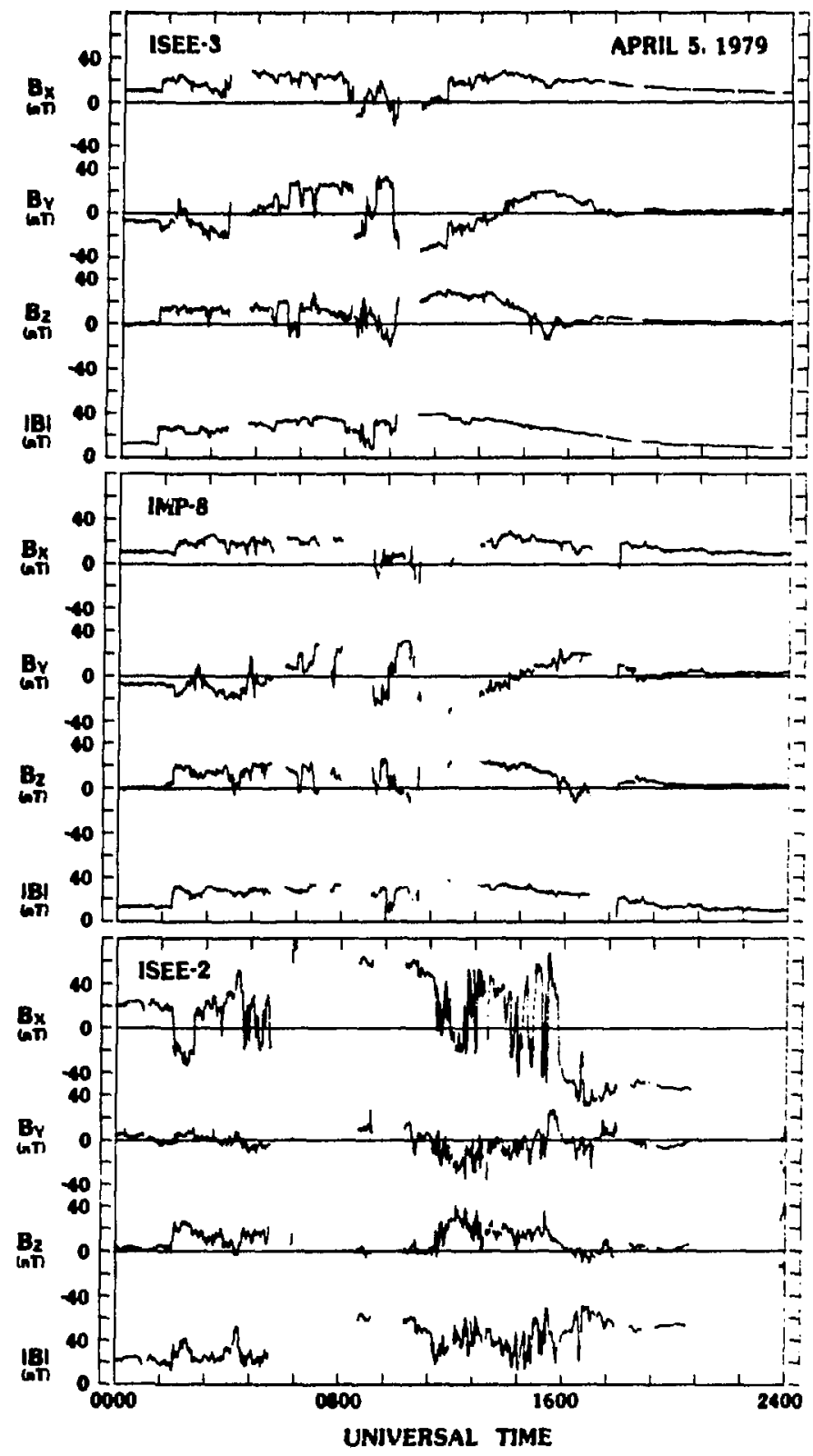

Fig. 2.4. From top to bottom the magnetic field data from ISEE-3, $220 \mathrm{R}_{\mathrm{E}}$ upstream in the solar wind, IMP-8, in the near-earth solar wind, and ISEE-2, near the midplane of the magnetotail current sheet, $\sim 18 R_{F}$ behind the earth. The arrival of the interplanetary shock is easily observable at ISEE-3 at $\sim 0120 \mathrm{UT}$, and $\sim 40$ minutes later near the earth. The ISEE-2 satellite $B_{\text {}}$ reversals indicate that the satellite made frequent current sheet traversals throughout the day. 
crossings, three particularly slmple and interesting crossings have been chosen for analysis.

Ion density, thermal energy temperature, and pressure data from the ISEE-2 Los Alamos/MPE Fast Plasma Experiment are shown in Figure 2.5, from 12:00-18:00 UT, on this day. Reglons 1dentifled by the cross hatching in the figure are based on the plasma parameters and greyscale spectrogram (where count rate as functions of angle and energy are indicated by plot intensity) representations of the data, and were arrived at Independently from the magnetometer data. Plasma sheet Intervals are easily discernible in the greyscale spectrograms, and are Indicated in the moments by periods of enhanced plasma pressure. The magnetic field data (FIg. 2.4) shows reglons of roughly constant fleld strength on elther side of the current sheet crossings, while the plasma data Indicates that the ISEE-2 satelite continued to be enveloped within the plasma sheet throughout much of the interval. This comparison between the two data sets Indicates that the plasma sheet traditionally defined observationally from the plasma data [Bame et al., 1966, 1967] as the region of enhanced particle populations, and the cross tal1 current sheet defined as the reglon of substantially enhanced current density, are not equivalent. Thts difference w111 be examined In greater detal1 in Section 2.5 .

Rapld and smooth crossings of the geotall current sheet are relatively uncommon in the ISEE data sets, and are most often assoclated with geomagnetic storm and substorm activity. The crossings studied here, however, were specifically chosen to be 


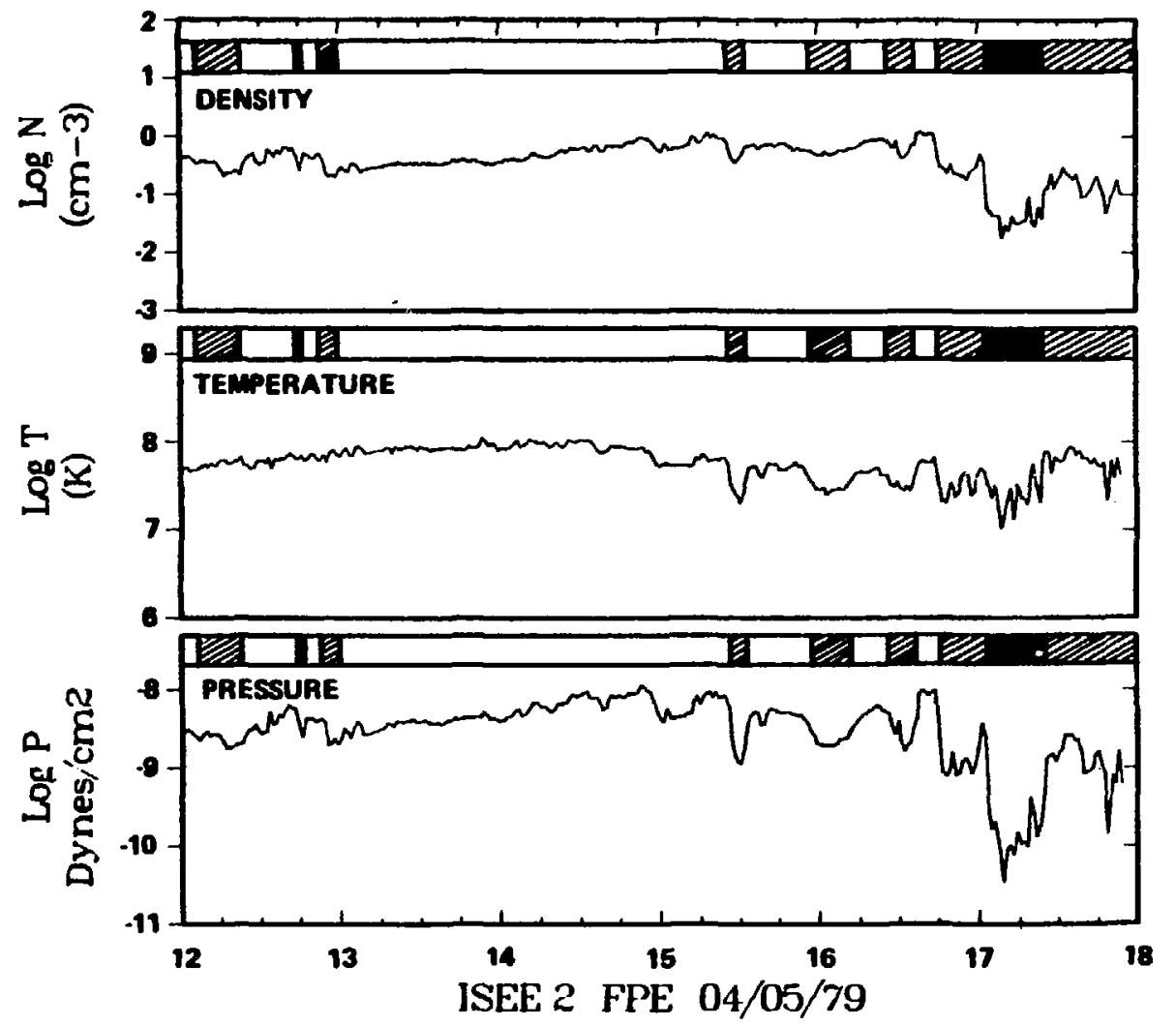

Fig. 2.5. Plasma moments at the ISEE-2 satellite for the interval from 1200 to 1800 UT. Regions identified in the panels are based on examinations of plasma greyscale and moments, and were arrived at independently from the magnetic field data. White indicator bars demark periods of plasma sheet, crosshatched bars indicate plasma sheet boundary layer and regions of uncertain plasma sheet determination, and the black region indicates the tail lobe. The satellite was clearly within the plasma sheet throughout much of the interval, even during periods when the magnetic fleld was relatively constant and not reversing. 
assoclated with bulk tall motions and not internal, substorm related tall reconfigurations. The z-component of the IMF was approximately zero for fourteen hours prior to the shock arrival and the $A E$ Index was small, Indicating that the crossing at approximately 2:00 UT was not due to a substorm. For the crossings at about $15: 15$ and 15:50 UT, the IMF had been substantially northward, Indicating that substantial magnetic flux was not belng added to the magnetotall, for at least two and one half hours prior to each of these encounters. Such a timescale is sufficiently larger than the Internal timescales of substorms (e.g., Foster et al. [1971]; Baker et a1. [1981]; Bargatze et al. [1985] that these crossings are probably not substorm related. The AE index for this later interval Is best described as disturbed (100-500 nT), but is apparently assoclated with the bulk reorlentations of the tail as a whole, and not Internal reconfigurations, as evidenced by a lack of classic substorm signatures in the $A E$ index from 00:00-06:00 and $12: 00-16: 00 \mathrm{UT}$.

\subsubsection{5:50 UT 5 Apr11 1979}

The simple sheet geometry described in the previous sections is appropriate to the crossing at $15: 50$ UT. This crossing has already been used to demonstrate the techniques for removing a variable sheet normal velocity, as shown in Figure 2.3. The top panel of Figure 2.3 shows the two satelite crossing signatures in their respective field line normal coordinate systems. In this case the Independently derived normals agree to within $8^{\circ}$ which indicates 
that the assumption of a simple, locally planar sheet geometry is falrly good. During the period from $14: 20$ to $15: 40$ UT the IMF configuration had both positive $\mathrm{B}_{X^{-}}$and $\mathrm{B}_{Y}$-components, which is in the sense opposite to the normal Parker spiral. Th1s configuration, however, is in the appropriate sense to give the observed $\mathrm{B}_{\mathrm{Y}}$-component in the tall for the mapping of the IMF $\mathrm{Y}$-component into the magnetotail, as has been statistically demonstrated by Fairfield [1979] and Lu1 [1983].

At 15:50 UT the satellites were located near the center of the magnetotail at $(-17.6,-3.2,0.2) R_{E}$ GSM (ISEE-1) with a separation vector $S_{\sim}=(-6826,1241,2732) \mathrm{km}$, GSM. The L-axes derived from minimum varlance analysis are $\hat{\mathrm{L}}_{1}=(.991,-.008, .134)$ and $\hat{\mathrm{L}}_{2}=(.975, .092, .202)$ for ISEE-1 and 2 , respectively, in GSM coordinates. The small $\mathrm{Y}$-component of the L-axes is common for field lines near the center of the tail. The $X$ - and $Z$-components Indicate that the sheet is tilted upward toward the earth by $8^{\circ}$ and $12^{\circ}$ at the two satellites. The nominal sheet normals, $\mathrm{N}_{\mathrm{NOM}}$, for these L-axes, calculated as described previously, are $\hat{\mathrm{N}}_{\text {NOM1 }}=(-.134, .001, .991)$ and $\hat{\mathrm{N}}_{\text {NOM2 }}=(-.201,-.019, .979)$.

The current density profiles shown in Figure 2.6 have been calculated assuming these nominal sheet normals. While the absolute magnitudes of the current densities and thicknesses of the current peaks are a function of the choice of the sheet normal, the relative distribution of the features is not. The effect of choosing a different current sheet normal is to inversely vary the calculated sheet thickness and current density magnitude. Since the total 


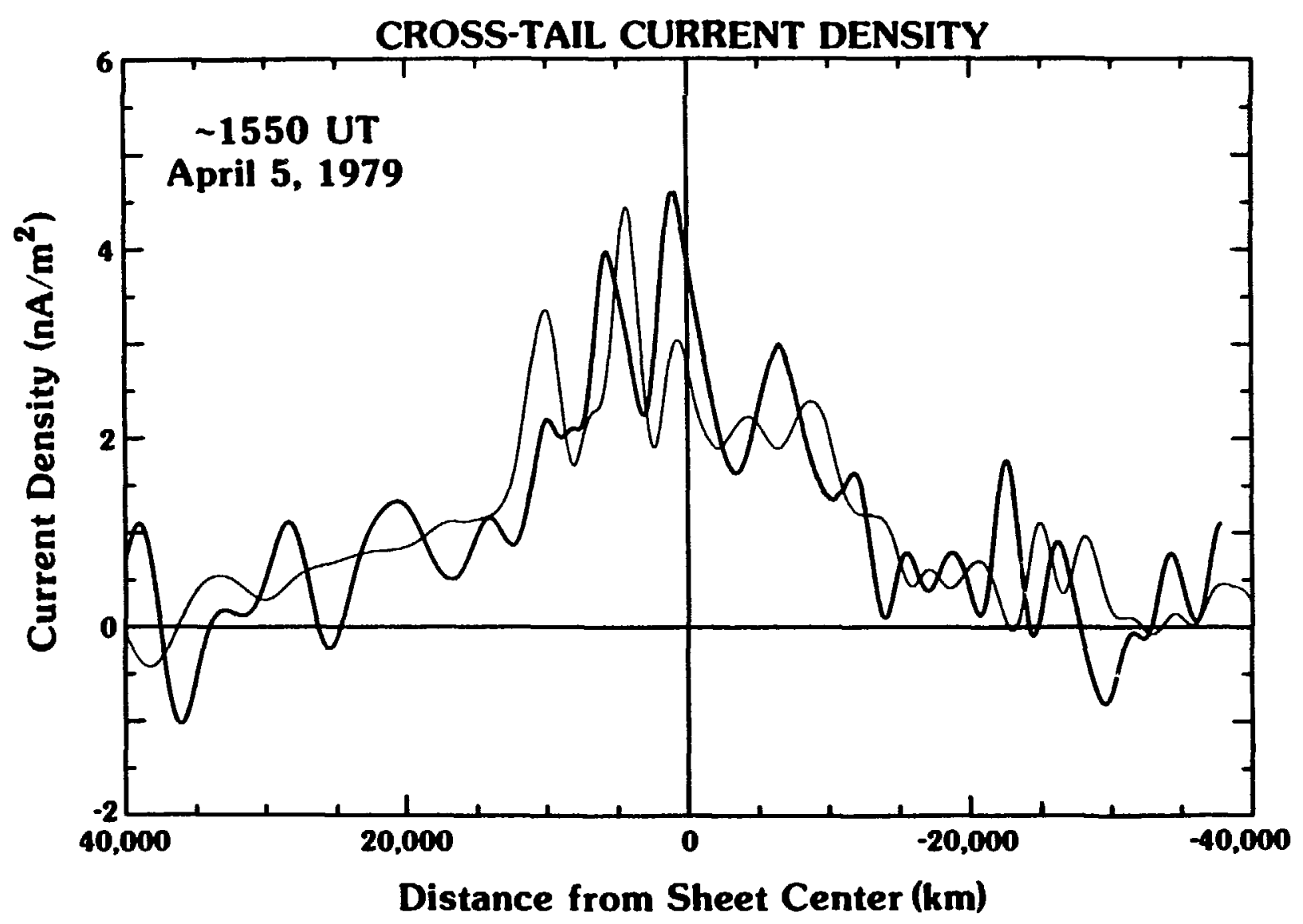

Fig. 2.6. Cross tail current density distributions for ISEE-1 and -2 displayed as a function of distance from the current sheet midplane for the crossing at $\sim 1550 \mathrm{UT}$ at $-17.6,-3.2,0.2 \mathrm{R} G \mathrm{GS}$. The distributions show the sheet to consist of a central current density enhancement surrounded on either side by lower density "shoulders." Further, the sheet is highly structured, the narrow density peaks being a fraction of an $R_{E}$ thick, and variable. 
sheet current is fixed, a thicker sheet requires proportionally smaller current densities throughout the sheet.

For this particular crossing the geometry of the two satellites is fortultous in that the separation vector between the satellites In plane $P$, namely $S^{*}$, Is only 22 degrees from the nominal normal. The maximum possible thickness, $\mathrm{T}_{\mathrm{MAX}}$, is therefore only $8 \%$ greater than the nominal thickness, $\mathrm{T}_{\text {NOM }}{ }^{*}$ This difference is of the order of other uncertainties in the calculation. Further, the field line normal, $n$, makes an angle of only 15 degrees with respect to $\mathrm{N}_{\text {NOM }}$, and is toward $S^{*}$. This angle ylelds a thickness only $3 \%$ different from $T_{N O M}$, and 1 s therefore even more negligible. Due to the unusually fortunate satellite/current sheet/field line geometry for this crossing, the calculated thickness and current density magnitudes are very 11kely to be correct. of course 1 is possible that the sheet is thinner than derived here, but there is no physical motivation to assume that the sheet should be excessively rotated from its nominal configuration, particularly given the field IIne orientations.

The current density distribution shown in Figure 2.6 is comprised of two rather distinct regions. The majority of the sheet current is carried in a central structure which is approximately $25,000 \mathrm{~km}$ thick, and which is embedded in a larger region of somewhat smaller and more uniform current density. This broader region is approximately $70,000 \mathrm{~km}$ thick although the actual edges are not particularly well-defined. These thicknesses can also be described in terms of thermal lor-gyroradil. Using the asymptotic 
plasma parameters ylelds thicknesses of $\sim 145$ and $\sim 407 \mathrm{R}_{1 \mathrm{~g}}$, and using central sheet plasma parameters ylelds $\sim 60$ and $\sim 169 \mathrm{R}_{1 \mathrm{~g}}$ for the thin central, and broader regions, respectively. While 1 t may be tempting to call this broad current region the plasma sheet, it must be noted that the plasma data both preceding and following this crossing indicate that at least ISEE-2 was located within the plasma sheet continuously. Not only does Figure 2.6 demonstrate that the bulk of the sheet current is carried in a central layer that is thin compared to the overall width of the plasma sheet, but it further shows a tremendous amount of small scale structure.

Fluctuations in current density are found throughout the entire current sheet, at least at the time of this crossing. Typlcally, the variations occur on size scales of about $5000 \mathrm{~km}$ ( 29 asymptotic or 12 central $R_{1 g}$ ) for this crossing, which is consistent with, or somewhat larger than, the minimum resolvable size scale from the intersatelite timing. Since the magnetic field data were filtered to remove variations with frequencles higher than the inverse of the intersatelite timing, structures observed in these distributions cannot be accounted for by simple motions of a planar, parallel current sheet. The fact that small scale variations observed at ISEE-1 and -2 do not agree further demonstrates that the sheet cannot possess a parallel and planar structure on the size scale of the satellite separation vector. This 1mportant observation will be examined in greater detail in the Discussion section of this chapter.

Plasma data from the Los Alamos/MPE Fast Plasma Experiment 
onboard ISEE-2 are shown in Figure 2.7 for the Interval from 15:00-16:00 UT. During the first half of the crossing from approximately 15:43:45 to 15:48:30 (1ndicated by the first palr of vertical 1ines) the proton temperature is slightly below its presheet value while the number density is silghtly above its presheet value. During the second portion of the crossing from approximately 15:48:30 to 15:53:30 these two parameters switch, with the density dropping below and the temperature rising above their orlgiral values. The total lon plasma pressure throughout this crossing is surprisingly flat, and shows only a small decline during the course of the crossing. The electron pressure (not shown), which is typlca11y $10 \%$ of the Ion pressure, simflarly shows 11ttle variation across the sheet. For an observed plasma pressure of $\sim 5 \times 10^{-9}$ Dynes $/ \mathrm{cm}^{2}$, the beta of the plasma vartes from $\sim 1$ at the edges of the sheet to $\sim 4$ at the center. The variation required in the plasma pressure to achleve a condition of hydrostatic equilf.brium should be resolvable in the plasma data. This condition has been examined for this crossing, and $J \times B$ and $\nabla p$ are found to be In agreement only to an order of magnitude. It therefore seems unlikely that the current sheet could be in a true state of hydrostatic equilibrium at the time of this crossing.

Also of interest is the determination of the three-dimensional plasma flow velocity vector. Figure 2.8 shows the plasma flow velocity vector components and magnitude in the GSM coordinate system. Polnts which show total velocities of less than about 40 $\mathrm{km} / \mathrm{sec}$ are not rellably determined and should not be considered to 


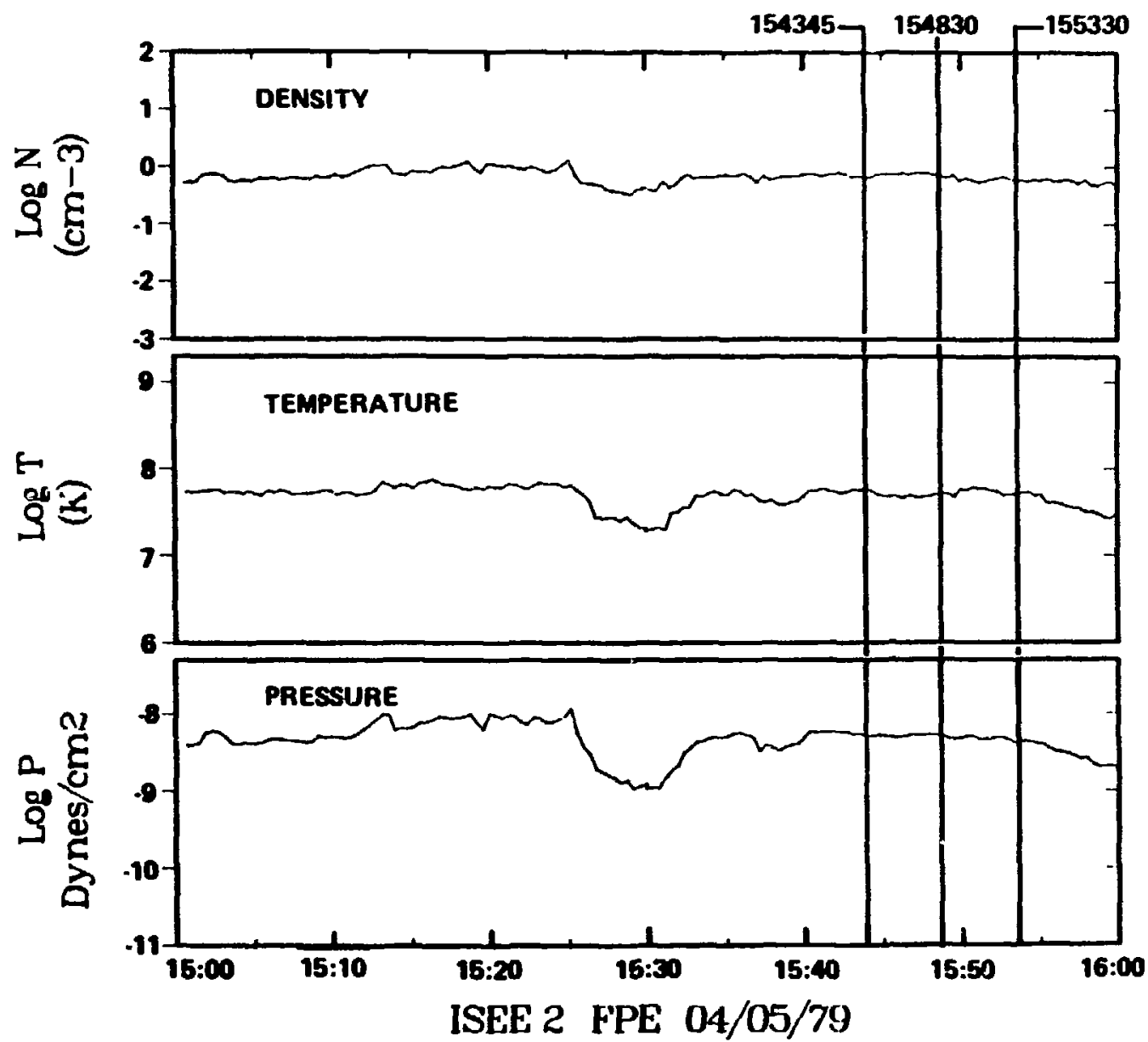

Fig. 2.7. Proton density, temperature, and pressure for the interval from 1500 to 1600 UT, at ISEE-2. The three vertical lines indicate the center of the sheet crossing and $\pm 40,000 \mathrm{~km}$. 


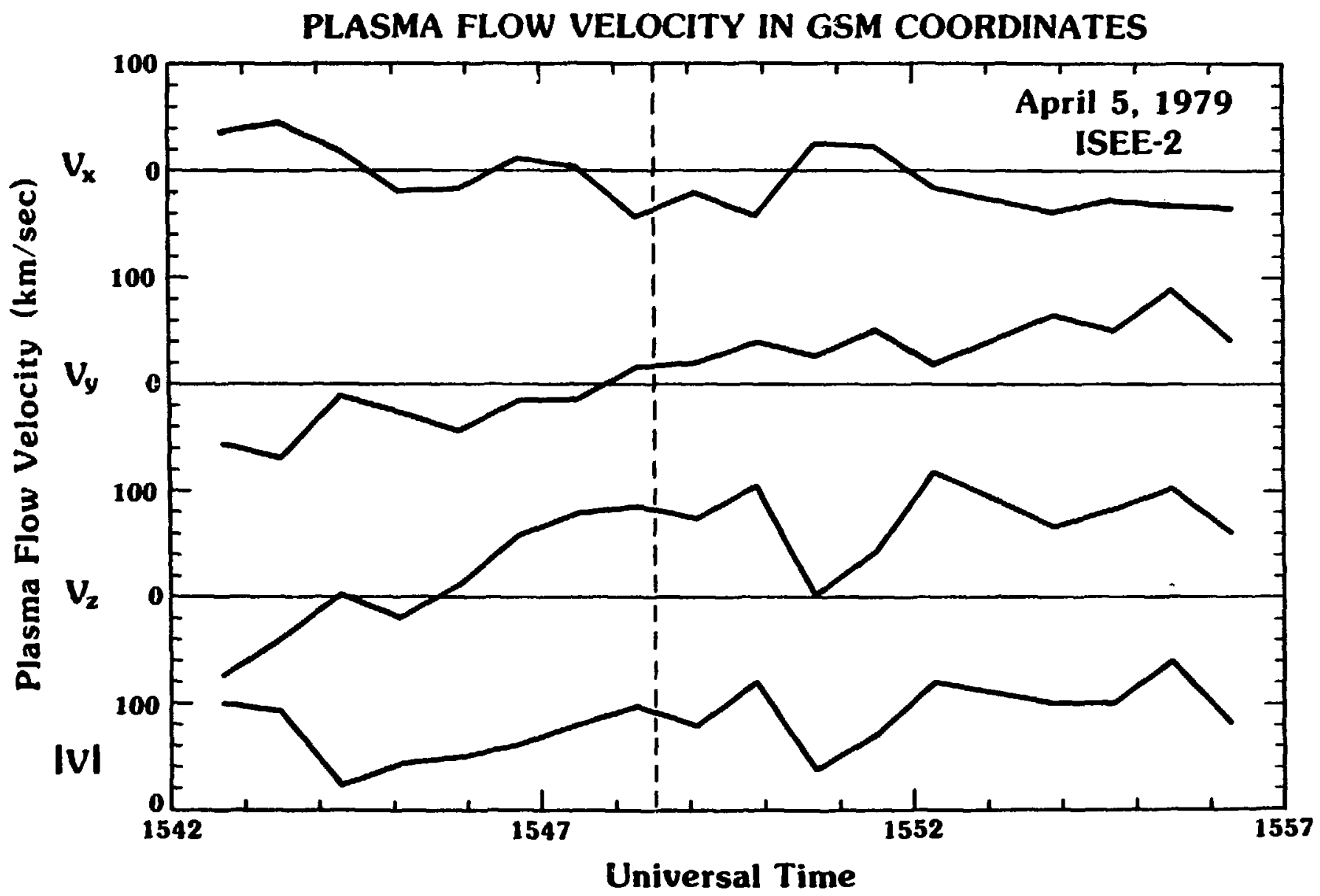

Fig. 2.8. Three dimensional plasma velocity components in GSM coordinates. Velocities at times when the magnitude is less than about $40 \mathrm{~km} / \mathrm{sec}$ are not reliably determined. The predominantly upward velocity of the plasma is consistent with the derived sheet motion. 
be significant. The vertical dashed 11ne indicates the center of this sheet crossing. The $\mathrm{z}$-component of the plasma velocity is positive and relatively large throughout most of the crossing. This direction is consistent with the upward motion of the current sheet required to account for a crossing from the north (earthward fleld) to the south (tallward fleld) side of the sheet. Further, the approximate magnitude of the upward plasma motion, while not in perfect agreement with the intersatellite timing derived velocity, 1s also not 1nconsistent with 1 t. The $X$ - and Y-components of the plasma velocity remain generally quite small throughout the sheet crossing. While the $\mathrm{X}$-component crosses back and forth between sma11 positive and negative values, the $Y$-directed velocity shows a marked asymmetry between the two sides of the sheet.

Flows on the north side of the sheet appear to be predominantly from dusk to dawn while flows on the south side are entirely from dawn to dusk. The expected direction of proton motion based on ion gyromotion back and forth across the fleld reversed sheet is from dawn to dusk for both sides since the magnetic field $X$-component changes sign across the sheet midplane. Observed asymmetries in this flow are at present not well understood and could simply be due to small errors in the choice of coordinate systems. In any case, such asymmetries are not of primary interest for the purposes of th1s study. Plasma flow vectors are shown here principally to corroborate the intersatellite timing derived motion of the sheet.

Electric field data (supplied by C. Cattell from the ISEE-2 electric field detector) for this crossing have been examined to 
determine if they are also consistent with the observed sheet motions, Since the z-component of the electric field is not avallable throughout most of the crossing, due to experimental limitations, a rotation of the data from the satellite to the GSM coordinate system is not possible. The two systems, however, only differ by a rotation about their common $\mathrm{X}$-axis by 20 degrees. It is therefore possible to look for at least a qualitative agreement between $-\mathrm{v} \times \mathrm{B}$ and the actual measured electric fields. The $\mathrm{Y}-$ component of the electric field is predominantly negative through the first half of the crossing, and positive through the second half, with a brief positive excursion at the start of the crossing. Since $B_{X}$ is positive in the first half of the crossing, and negative in the second half, a plasma flow which starts first negative and then goes positive at about 15:45 is entirely consistent with the observed electric field Y-component, further substantiating the observed plasma and sheet velocities.

2.4.2 02:00 UT 5 Apr11 1979

The 1nterplanetary shock which arrived at the earth fust prior to 2:00 UT on 5 April 1979 and the region of enhanced plasma pressure which followed tt have already been discussed. The Inttial tail current sheet crossing at about 2:00 is almost surely a direct consequence of the Inttial impact of the interplanetary shock as it occurs at ISEE-1, about $22 R_{E}$ back in the tail, less than 10 minutes after the passage of the shock at IMP-8 $(-2,22,27) R_{E} G S M$. The approximate shock normal derived from the magnetic coplanarity 
theorem is $(-.69, .42, .59)$ and the velocity 1ncreases across the shock by $>100 \mathrm{~km} / \mathrm{s}$ at ISEE-3. Th1s ylelds an expected 1nitial upward motion of the magnetotall. The crossing at the ISEE satelittes is from the north to the south side of the sheet as would be expected for an upward tall motion. Th1s case represents a geomagnetically moderate sheet crossing since the IMF $B_{Z}$-component was approximately zero for fourteen hours prior to the shock, and the AE Index was less than $300 \mathrm{nT}$ and relatively smooth during the twelve preceding hours.

F1gure 2.9 displays the magnet1c field data, calculated normal velocity, and current density distribution for this crossing which occurred at $-22.0,-1.4,4.0 \mathrm{R}_{E}$ GSM (ISEE-1). The calculations used to obtain the values shown in the lower two panels assume the nomina1 normal as was done previously. In this case these normals are $\hat{\mathrm{N}}_{\mathrm{NOM}}=(.217, .052, .975)$ and $\hat{\mathrm{N}}_{\mathrm{NOM} 2}=(.197, .061, .979)$ and agree to within $4.4^{\circ}$, Indicating the appropriateness of the assumption of a flat current sheet on the Intersatelite separation size scale. The top panel shows the original magnetic fleld data in the GSM coordinate system. The dashed vertical 11nes show which portions of the data correspond to the central region of enhanced current density in the bottom panel. This mapping is indicated for both ISEE-1 and ISEE-2 with the set of four 11nes, and further emphasizes that locations in the sheet correspond to very different times at the two satellites.

It is interesting to note the marked asymmetry in the magnetic fleld profiles. While the variation of the $\mathrm{X}$-component of the fleld 


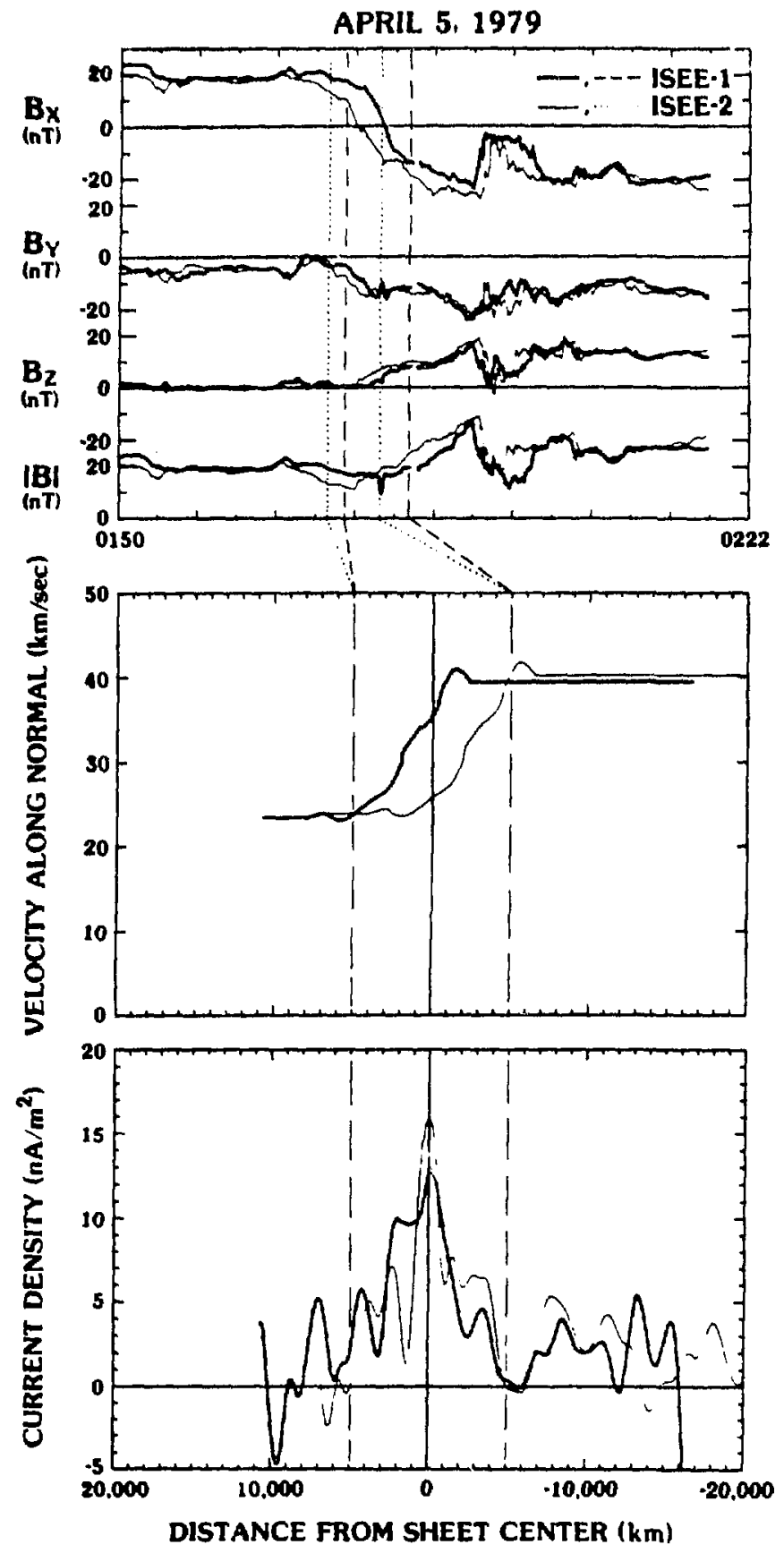

Fig. 2.9. Magnetic field, sheet normal velocities, and current density distributions for the sheet crossing, at $-22.0,-1.4,4.0$ $R_{E} G S M$, associated with the Initial arrival of the interplanetary shock at 0200 UT. The central current density enhancement is indicated by the dashed vertical lines in the bottom two panels, and mapped up to the appropriate portions of the magnetic signatures for the two satellites independently. 
is reasonably symmetric, the $\mathrm{Y}$-component decreases and the $\mathrm{Z}-$ component increases, both rather unfformly through the crossing. In the coordinate systems used in this study, all these variations end up in the $B_{L}$-component, and the current is driven parallel tr, the $M-$ axis. The net effect is that the total field sirength aecreases very little through the first half of the crossing, and increases very rapidly through the second half. These profiles indicate that the magnetic pressure in the tail is increasing during the period of crossing as would be expected if the tall is being compressed by the arrival of the shock. While this variation technically contradicts the stated assumption of a sheet which is not varying on the timescale of the crossing, it is still posstble to analyze this crossing by assuming that the sheet does not vary much on the intersatelilte timing timescale, and that the principal effect of the pressure increase is simply to compress the sheet isotropically. The middle panel of Figure 2.9 shows the normal velocity for this case and was derived. from filtered, rotated data, just as in the previous example. The sheet normal was again chosen to be the nominal normal given the derived L-axis orientation; however, the normal velocity values derived are appreciably slower than before. In the lower panel, the calculated current density is displayed. The thickness of the central enhanced region is about $10,000 \mathrm{~km}$ (35 asymptotic or 15 central $R_{1 g}$ ), and the peak current density is in excess of $15 \mathrm{nA} / \mathrm{m}^{2}$ at ISEE-2. Both the satellite signatures show a narrow central current density peak surrounded on either side by somewhat smaller current density "shoulders" in the distribution. 
The regions outside of about $\pm 5,000 \mathrm{~km}$ show a net positive current density with an appreclably smaller average value than the central structure. While 1 t is not possible to identify these regions unambiguously with the broad, low current density regions observed In the first case study, the obvious simflaritles at least make such an Identification plausible.

An Interesting major difference between this case and the previous one is that the fle!d lines in this current sheet have a large cross tail component. The planes which contain the field line rotations are in fact inclined by $74^{\circ}$ and $71^{\circ}$ fron the ISEE-1 and -2 most nominal normals respectively. Magnetic field lines in this sheet crossing are therefore strongly directed in the cross tall sense. The IMF direction prior to the arrival of the shock is in the normal spiral direction for an in-pointing sector, and the strong cross tall component of the fleld in the sheet is from dusk to dawn, as would be expected if a portion of the Y-component IMF was mapped 1nto the tall. Geometrles for the current sheet structures which are consistent with the observation of such a large fleld line Inclination include a relatively nominal sheet orientation with highly inclined fleld lines in 1t, a sheet which is locally very wavy with reasonably perpendicular fleld line orlentations inside, or some intermediate configuration between these. It is extremely unlikely that the entire current sheet could be rotated by some $70^{\circ}$ this close to the earth; however, it is possible that the sheet could be very wavy or lumpy and therefore could have sections which have locally inclined normals of $70^{\circ}$ or 
more. If the fleld lines are actually normal to the sheet surface, as In the wavy current sheet picture suggested here, then the current density distribution and sheet normal velocity shown in FIgure 2.9 would have to be rescaled. The central current sheet structure thickness would drop from about $10,000 \mathrm{~km}$ to $4,000 \mathrm{~km}$ and the peak ISEE-2 current density would rise to over $35 \mathrm{nA} / \mathrm{m}^{2}$. Such a current density is not unreasonable, and the questions of the actual sheet orlentation and maximum current densities will be examined in greater detall in Section 2.5 .

The satelite separation vector for this crossing is $(-573,1503,2389) \mathrm{km}$ in GSM coordinates. The component of this vector In the plane normal to the $L-a x 1 s, S^{*}=(76,1656,2220) \mathrm{km}$. The scaling factor for the sheet maximuin thickness and minimum current density is therefore on 1 y 1.2 . Th1s means that the greatest thickness that the nominally $10,000 \mathrm{~km}$ thlck central structure could be $1 \mathrm{~s}$ about $12,000 \mathrm{~km}$ (42 asymptot1c or 18 central $\mathrm{R}_{\mathbf{1 g}}$ ), and the peak ISEE-2 current density must exceed $12 \mathrm{nA} / \mathrm{m}^{2}$. As before, this case represents a somewhat fortunate satelifte orlentation in that the firm upper bound on the sheet thickness is quite close to the nominally derived value.

Three-dimensional plasma data for this interval indicates that the proton temperature, and to a lesser extent the number density, rises during the first half of the crosing and falls during the second half. This yields a plasma pressure profile in good qualitative agreement with the field diamagnetic reduction and a hydrostatic equilibrium condition. The plasma pressure on the south 
(exit) side of the sheet $1 \mathrm{~s} \sim 2.5 \times 10^{-9} \mathrm{NT} / \mathrm{m}^{2}$, which is about a factor of two higher than on the north (entry) side. Th1s varlation Is consistent with an increase in the pressure inside the tall during the course of the crossing as was irdicated in the previous discussion of the top panel. The plasma flow direction observed through the crossing is predominantly earthward and the bulk speed Is quite high, ranglng up to about $360 \mathrm{~km} / \mathrm{sec}$ during the second half of the crossing. Throughout much of the crossing the plasma flow vector points downward, in the opposite sense to achieve the observed north to south traversal. The magnitude of $v_{Z}$, however, is smaller than $v_{X}$ typlcally by a factor of about four. Since the current sheet is inclined with the normal having a negative Xcomponent, earthward motion of the sheet would be expected to carry the satelites from the nortin to the south. Further, errors in the three-dimensional velocities on the order of $30 \%$ are not unreasonable. The plasma flow data, therefore, cannot be constdered to be Inconsistent with the other observations of the crossing. Such large plasma flow veloctties are not expected in the cross tall current sheet at geomagnetically quiet times, and argue that this crossing occurs during a more active interval.

\subsubsection{5:15 UT 5 Apr11 1979}

The last 5 Apr11 1979 crossing studted here occurred at about 15:15 UT at $-17.9,-3.1,0.4 \mathrm{R}_{\mathrm{E}}$ GSM (ISEE-1). The crossing carried the satellites from the northern to the southern side of the sheet as shown in the top panel of Figure 2.10. It is clear from the 
APRIL 5, 1979
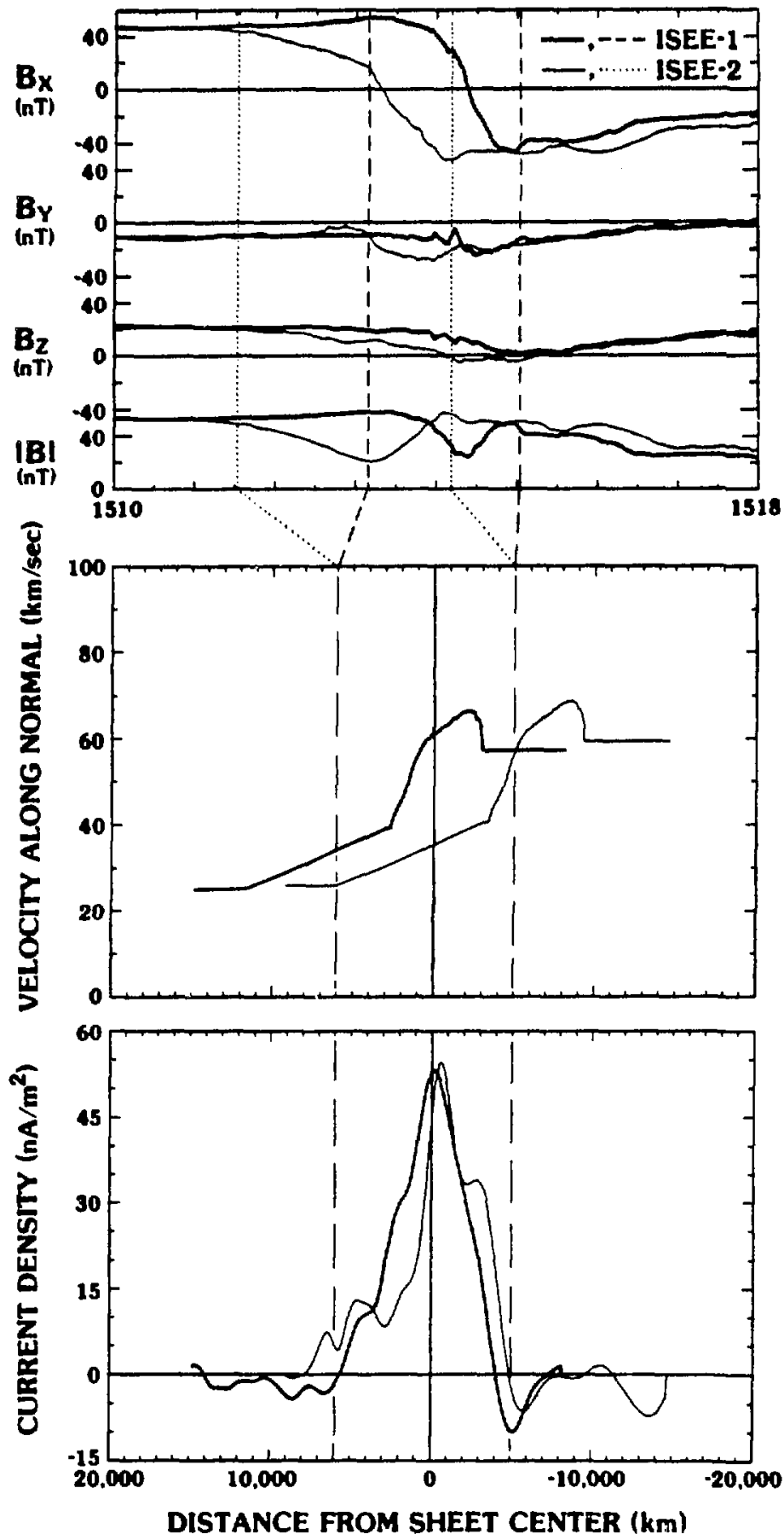

Fig. 2.10. Data for another crossing at 1515 UT and at $-17.9,-3.1$, $0.4 \mathrm{R}_{\mathrm{E}} \mathrm{GSM}$, in the same format as in the previous Figure. Note that the observed current densities are much greater than for the previous cases (see text). 
fleld data that the sheet normal velocity increases with time across the sheet, since the duration of the sheet entry is much greater than the duration of the sheet exit. Th1s observation is borne out quantitatively in the middle panel of Figure 2.10 which shows the actual sheet normal velocity. Both of the bottom two pane1s are based on the nominal normal sheet coordinates, as were the previous cases. Sheet normals derived independently for the two satellite data sets agree to within $5.2^{\circ}$, again indicating a locally flat sheet topology.

The bottom panel of this figure displays the calculated current sheet current densities as a function of location in the sheet, under the assumption of the nominal normal, as for the previous examples. Again, a relatively narrow current density peak is observed in the center of the sheet. The thickness of this structure is approximately $10,000 \mathrm{~km}(78$ asymptotic or 27 central $R_{1 g}$ ), and the peak current density is over $50 \mathrm{nA} / \mathrm{m}^{2}$. This value $1 \mathrm{~s}$ appreciably larger than those measured for the previous two examples, but the full sheet thickness is also narrower than previously observed. For this case, unlike the previous examples, no regions of somewhat smaller current density are observed at the edges of the strong central structure.

Fleld line derived axes yleld normals, which are rotated from the nominal normals, $(-.152,-.018, .988)$ and $(-.178,-.038, .983)$, to directions of $(.019,-.845, .535)$ and $(.079,-.877, .471)$, or through angles of $57^{\circ}$ and $61^{\circ}$ for ISEE-1 and -2 , respectively. The $B_{Y}$-component of the field is small, but in a sense inconsistent with 
the expected sense of the IMF at the time of this crossing. This might be accounted for, however, by the fact that the IMF $B_{Y}$ had changed direction fust one hour prior to this crossing and the timescales for reversing the sense of lonospheric rotation and tall $\mathrm{B}_{\mathrm{Y}}$ could be of this order. Calculations based on the assumpition that the fleld rotates in a plane normal to the sheet surface give the unbellevable results. of a sheet thickness of only $440 \mathrm{~km}$, and maximum current density of $1200 \mathrm{nA} / \mathrm{m}^{2}$. It therefore seems 11kely that the planes containing the magnetic fleld line rotations in the sheet are substantially inclined with respect to the sheet normal 1tself. Calculation of the maximum sheet thickness based on the orlentation of the separation vector in the sheet, $s^{*}$, yields a thickness which is on $1 y 20 \%$ larger than that derived for the nominal normal. The firm upper bound on the current sheet thickness for this case is therefore only $12,000 \mathrm{~km}$ ( 94 asymptotlc or 32 central $\left.R_{1 g}\right)$

Plasma data for this crossing, also shown in Figure 2.7, Indicate the qualitatively expected Increase in plasma pressure for hydrostatic equilibrium. It is not possible to examine this equilibrium in detall since the entire crossing took only about 2-3 minutes, which gives only a few plasma spectra. Plasma flow vectors, while also inconclusive due to the very short crossing duration, show that the flow in the sheet is princlpally dawn to dusk with only small possible earthward and upward components. Such a flow is consistent with the slow crossing speeds displayed in 
Figure 2.10 and with the north to south nature of the crossing, since the errors in measuring very slow flows are large.

\subsection{Discussion and Summary}

Three current sheet crossings associated with the reglon of varying solar wind pressure behind an Interplanetary shock on 5 Apr11 1979 have been studied in detail. One of the three is apparently associated with the initial shock arrival, while the other two occur much later in the day. The effect of the Initial shock and assoclated region of varying solar wind pressure behind $1 t$ Is to compress and deflect the magnetotall. As a consequence, the ISEE-1 and -2 satellites, whtch were orbiting near the midplane of the magnetota11, repeatedly encountered the cross tall current sheet. The crossings studied here, whlle not representative of the configuration of the quiescent current sheet, do correspond to relatively quick and analyzable crossings that are probably not principally due to substorms and the major internal magnetotall reconfigurations assoclated with them. The current sheet crossings studied here, while best described as occurring during a disturbed perlod, are probably as close to the qulet tall configuration as are any crossings which are feasible to analyze, even with our technique.

Throughout most of the 19 hour post-interplanetary shock perlod, the ISEE-2 satellite was engulfed in the magnetotall plasma sheet as evidenced by plasma sheet greyscale and plasma parameters. During this perlod the ISEE satellites made repeated encounters with 
the current sheet. These encounters, however, were separated by relatively long perlods when the satellites were apparently not in regions of substantially enhanced current density, since $\underline{B}$ was relatively constant. This observation is consistent with the usual theory of the near earth magnetotall which defines the plasma sheet as the region of enhanced plasma density associated with the particle populations trapped on closed fleld lines. The current sheet, however, is defined by the major rotation of the magnetic fleld between roughly sunward and antisunward, and the associated cross tall currents. The current sheet topologically must 11e Inside the plasma sheet since the current sheet in the near tail 18 assoclated with the curl of closed field lines, but the region of substantial current density need not fill the plasma sheet region, and may be simply embedded in 1t. This possible difference between an Intrinsic magnetotal's current and plasma sheets is in contrast to the structures of intrinsic magnetotalls downstream from an $x-$ line, and Induced magnetotalls, neither of which have regions of closed fleld Iines to trap particle populations.

While a clear difference between current and plasma sheet definitions has been delineated in the near magnetotall region, it should not be concluded that no cross tall current is present in the plasma sheet region outside the central current sheet. It seems 11kely that the entire plasma sheet region carries some current, while the central current sheet is simply the enhanced region which carries the bulk of the current.

The general current sheet geometry has been examined and the 
1aherent difficulties of resolving a three-dimensional structure with data from only two satellites have been discussed. Magnetic fleld rotations through the sheet were seen to occur in planes which were inclined with respect to the nominal sheet configurations. The simplifications possible because of the well-defined nature of this maximum variance axis and assumption of planarity, greatly reduce the ambiguity of the geometry so that the sheet geometry is fully resolved to within one missing plece of information. This missing information 1s an angle between the profections of any of the known vectors Into the plane perpendicular to the L-axis, and the actual sheet normal vector. Without knowledge of this angle it is only possible to set upper bounds on the sheet thicknesses based on the chance orfentation of the satellite separation vector, and speculate as to the actual sheet orlentation.

In this study, data has been displayed in the nominal normal coordinate system in each case. This normal is along $Z_{G S M}$ if the Laxis colncides with GSM X. Small deviations of L from GSM X are due to small deflections in the tail position and the internal flaring of fleld lines away from the dipole field. The fact that local nominal normals independently derived at the two satellite agree to within $8^{\circ}, 5^{\circ}$, and $6^{\circ}$, respectively for the three cases, Indicates that the current sheet topologies are quite flat, at least in $x$, on the separation size scale of a few thousands of kflometers. It is unlikely that current sheet waviness could account for local Inclinations in the sheet of $70^{\circ}$ or $60^{\circ}$ as indicated by assuming the field lines are perpendicular to the sheet surface in the second and 
third cases. The radius of curvature of such structures, given that the sheet is flat to within about $5^{\circ}$ over a couple thousand kllometers in both cases, would have to be 3 to $4 R_{E}$. Such large scale waviness of the plasma sheet surface at substorm times has been reported previously by Forbes et al. [1982]; however, the observed waves were all of small amplitude (600-1400) km over wavelengths of $1-5 R_{E}$. Observed tilts at the reported crossing locations were less than $20^{\circ}$, and the very large t1lts required to explain the observations in this study seem very unlikely. Furthermore, the cases studled here do not occur at strongly geomagnetically active times when such large amplitude perturbations are probably somewhat more 11kely. A far more likely situation is that the sheet is approximately in 1 ts more nominal configuration and the field lines within it are simply highly inclined. Two of the three cases studied here exhibit large $\left(\geq 60^{\circ}\right)$ field line Inclination angles from the most nominal sheet normals" While this is certainly not a statistically significant number of cases, we can at least speculate that such large angles may not be uncommon in the cross tall current sheet.

One possible explanation for these large Inclinations has been described by Moses et a1. [1985]. They suggest that oppositely rotat!ng convection patterns of the open fleld lines in the two pola: caps can cause motions of the closed (plasma sheet) fleld In's which are in the sense to shear the sheet, and create large cross tall magnetic field components in the plasma sheet. For the first two cases described here, the IMF ${ }^{B_{Y}}$-component was in the 
appropriate sense for this effect to have caused the observed Y-component of the fleld in the plasma sheet. The third crossing occurred fust 45 minutes after the IMF had completed a 5.5 hour excursion with a peak $B_{Y}$ field $>30 \mathrm{nT}$, in the appropriate sense for the observed $Y$ fleld in the plasma sheet. None of the crossings studied here are inconsistent with the Moses et al. model if the timescale for changes of the $Y$ fleld component in the plasma sheet $1 \mathrm{~s} \geq 1$ hour. In any case, the derived upper bounds of $27,000 \mathrm{~km}$ and $12,000 \mathrm{~km}$ for the central structures in the first two cases, and $12,000 \mathrm{~km}$ total for the last, firmly fix the maximum sheet thickness for these cases. While the set of three crossings studied here in no way constitutes a statistically significant set, it is interesting to note that the two thinner, higher current density crossings are associated with the sheets which have large fleld line Incilinations.

Magnetometer and plasma analyzer data have been used together throughout this study to ald in the examination of the current sheet structure, and a number of points of contact have been made between these data. Three dimenslonal plasma flow velocities indicate that plasma motions observed in the sheet are consistent with sheet velocities derived from Intersatelite timing under the nominal normal assumption. This observation indicates that actual sheet normals are probably similar to the nominal ones derived here. Inverse varlations were found to exist between the magnetic and plasma pressures across the dlamagnetically reduced current sheet in two of the three cases. Further, in the 02:00 UT case, the 
pressure in the tall as a whole increased during the crossing, and the expected pressure variation was observed within the sheet as well as without, Indicating that, at least in this case, a rough state of pressure balance existed throughout the current sheet just as was shown for the plasma sheet by Fairfield et al. [1981]. Varlations from hydrostatic equilibrium cannot represent the stable, steady state conffguration of the sheet. Rather, they probably correspond to times when the tall orlentation is adjustirig to variations in the external solar wind pressure.

Two of the three crossings examined here showed similar structure: a large current density feature in the center of the sheet and smaller "shoulders" In the current density dietribution on one or both sides. In the third case, the data does not extend sufficlently far out to the sides to be sure whether shoulders exist or not. Since the calculation and removal of the variable velocity effect is only possible in the central portions of the sheets, and is simply extrapolated out from there, a lack of observed shoulders may simply indicate that current densities in the outer current sheet are not rellably determined. If the large central current structure is always surrounded by lower denefty shoulders, then this would be indicative of a current distribution which is described by a single function with a rather sharp cutoff. Such a sheet distribution would be consistent with a model which describes the fleld line rotation by a function which drops to low, but nonzero values away from the central current sheet. One such function for $B_{L}$ is the hyperbolic sine, while the planes of the fleld lines are 
inclined in the sheet so that both of the nonreversing fleld components have constant, nonzero values. In th1s case the cross tall current would be positive and slightly decreasing all the way out from the near shoulder regions to the edges of the plasma sheet. An Important result of this study is that, while the gross structure of the current density distribution is shown to consist of two relatively distinct regions, a substantial amount of fine structure is also shown to be present in the current density distributions. These structures are typically $2-6 \times 10^{3} \mathrm{~km}$ thick, which corres.ponds to tens of Ion gyrorad11, and are resolved in the data since it has been filtered to remove all variations on timescales shorter than the intersatellite timing timescale. The observed structures could represent compressional waves in the plasma sheet, real current fllaments, or breakdowns in the assumption of a plane parallel geometry which would agaln indicate spatial current structures. Structures observed in one satellite's current distribution are usually mirrored in the other's distribution although shifted in magnitude, and sometimes slightly in location. Such a correlation is consistent with a model in which the current s'seet is comprised of numerous elements of enhanced current density, or possibly current driven Instabilities. Although larger than would normally be expected, filamentary structures might be due to kinetic ion effects and single particle type motions. 
2.6 Chapter Acknowledgments

The authors gratefully acknowledge valuable discussions with J. Birn, J. T. Gosling, G. L. Stscoe, H. E. Spence, and G.-H. Voigt, and sincerely thank Phillip Lee for helping in the development of the variable velocity computer program which made this study possible. Much appreciated electric field data examined for one of the crossings was supplied by C. Cattell and the UCB instrument team. Magnetic field data from ISEE-3 and IMP-8 were obtained from the NSSDC through J. KIng. We thank the 1nstrument teams at JPL and GSFC for providing these data. This work was princlpally carried out under the auspices of the Institute of Geophysics and Planetary Physics, UCLA and supported under NASA contract NAS-5-28448. The principal author was supported by Los Alamos National Laboratory under the auspices of the Unfted States Department of Energy as a part of the Advanced Study Program, while research done at Los Alamos was supported under NASA contract S-04039. Finally, the authors wish to thank the referees, who supplied valuable suggestions for improving this chapter. 


\section{CHAPTER 3}

The Average Magnetic Fleld Draping and Consistent Plasma

Propertles of the Venus Magnetotall.

\subsection{Chapter Overview}

A new technique has been developed to determine the average structure of the Venus magnetotail (In the range from $-8 R_{y}$ to $-12 R_{V}$ ) from the Ploneer Venus magnetometer observations. The spacecraft position with respect to the cross tall current sheet is determined from an observed relationship between the field draping angle and the magnitude of the field referenced to its value in the nearby magnetosheath. This allows us to statistically remove the effects of tall flapping and variability of drapling for the first time, and thus to map the average field configuration in the venus tail. From this average configuration we calculate the cross tail current density distribution and $\underset{\sim}{\mathrm{J}} \times \mathrm{B}$ forces. Continuity of the tangential electrir field is utilized to determine the average variations of the $X$-directed velocity which is shown to vary from $-250 \mathrm{~km} / \mathrm{sec}$ at $-8 \mathrm{R}_{\mathrm{V}}$ to $-470 \mathrm{~km} / \mathrm{sec}$ at $-12 \mathrm{R}_{\mathrm{V}}$. From the calculated Jx $\mathrm{B}$ forces, plasma velocity, and MHD momentum equation the approximate plasma acceleration, density, and temperature in the Venus tall are determined. The derived ion density is approximately $\sim .07 \mathrm{p}^{+} / \mathrm{cm}^{3}\left(.005 \mathrm{O}^{+} / \mathrm{cm}^{3}\right)$ in the lobes and $\sim .9 \mathrm{p}^{+} / \mathrm{cm}^{3}\left(.06 \mathrm{O}^{+} / \mathrm{cm}^{3}\right)$ in the current sheet, while the derived approximate average plasma 
temperature for the tall is $\sim 6 \times 10^{6} \mathrm{~K}$ for a Hydrogen plasma or $\sim 9 \times 10^{7} \mathrm{~K}$ for an Oxygen plasma. Finally, the calculated plasma flow speed and density are combined to yield an upper bound on the flux of planetary lons down the Venus magnetotall, and give a value of $\sim 1 \times 10^{26} \mathrm{amu} / \mathrm{s}\left(\sim 6 \times 10^{24} 0^{+} / \mathrm{s}\right)$.

\subsection{Introduction}

Extensive In situ observations of the magnetic field and plasma populations in the Venus environs have a shown that 1) Venus does not have a significant intrinsic magnetic field [Russel1 et al., 1980a] and 2) a magnetotail is a regular feature of the region antisunward of the planet (Russe11 [1976]; Dolg1nov et al. [1978]; Russe11 et al. [1981], [1985]; Intr1l1gator and Scarf [1984]; Slavin et al. [1984]; Saunders and Russel1 [1986]). The Venus magnetotail is generally believed to form by magnetic field draping about the Venus lonosphere in a manner first suggested to expla1n comet tails by Alfuen [1957].

A steady state configuration of this interaction is shown schematically in Figure 3.1. The solar wind, with the embedded interplanetary magnetic fleld (IMF), flows radially outward from the rotating sun, causing the well-known Parker spiral pattern of the IMF. The solar wind carries the IMF flux through Venus bow shock and mass loading, extended, neutral exosphere, and then past the generally unmagnetized but conducting icrosphere. Little, if any, of the upstream plasma flow enters Venus lonosphere. Rather, the flow $1 s$ slowed, compressed, and deflected above the dayslde 


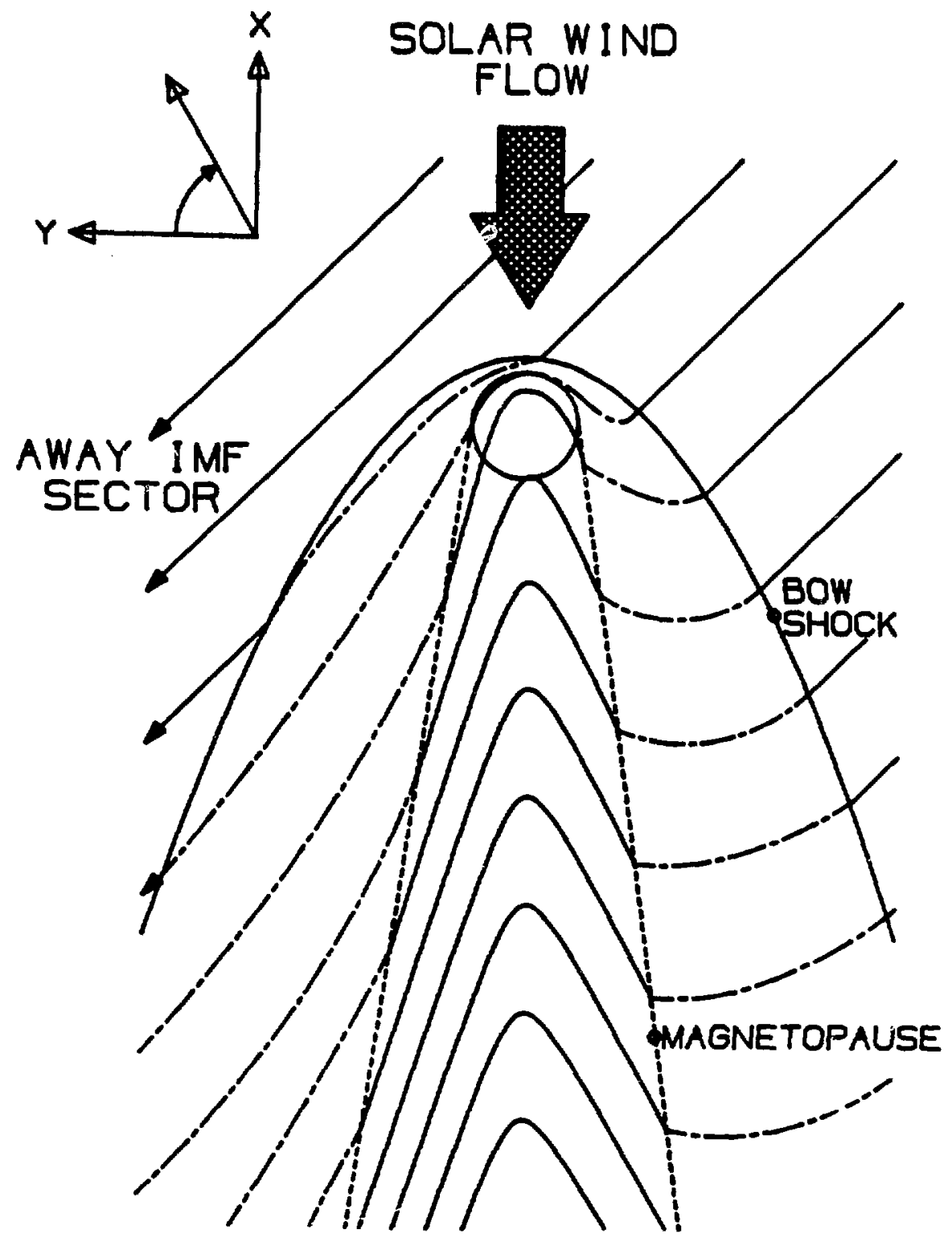

Fig. 3.1. Schematic diagram of the postulated solar wind interaction with Venus in the plane containing the upstream IMF. The upstream solar wind carries the IMF through the bow shock and magnetosheath. At the obstacle the magnetic field must flow perpendicular to this plane, and around the conductive ionosphere. This diversion of the flow, enhanced by mass loading of the flow near to the obstacle, causes the field lines to drape and form a magnetotail. Note also that the spiral urientation of the upstream magnetic field causes the magnetic flux in the left lobe to be greater than in the right at every distance down tail. This has important dynamical implications for the actual tail configuration (see text). 
Ionopause, and eventually slips around the planet. The plasma in the magnetosheath to the sides of the Venus obstacle and magnetota11 reglons travels at the larger velocity of the magnetosheath flow, which is a function of position behind the shock. Since the magnetic fleld lines link the magnetosheath and near Venus reglons, the lines become bent. This has the effect of draping the fleld Iines such that they are "hung up" on Venus and stretched out generally sunward and antisunward to the sides of the Venus magnetota11.

In addition to deflection of the flow by the conductive lonosphere viscous slowing of the flow by the lonosphere, and mass loading of the magnetosheath plasma with material from the extended Venus atmosphere and Ionosphere also contribute to Venus magnetota1l formation. The mass loaded plasma flow slows in order to conserve momentum, which significantly enhances the draping of fleld lines around the conducting lonosphere and substantially helps to produce the Venus magnetota11. Eventually, somewhere very far downstream, we expect that the field lines should once again assume their Interplanetary configuration due to the Maxwe1l stresses in the draped magnetic fleld configuration. These stresses should cause the kinked portions of the fleld lines to accelerate back up to, and beyond, the solar wind speed while mass loaded material tends to diffuse along the fleld lines due to its parallel plasma temperature.

There is now much direct and Indirect. evidence for mass loading of the Venus magnetosheath by the pick-up of newly created lons and 
other processes such as charge exchange. Oxygen Ions have been seen in the near terminator region of the Venus magnetosheath [Mihalov and Barnes, 1981] and occasionally in the distant magnetosheath and magnetotall region [Mihalov and Barnes, 1982]. An enhancement of the magnetosheath magnetic fleld strength has been found in the hemisphere where most ion mass plck-up is expected, presumably due to a decrease of the magnetosheath flow speed and the consequent pile-up of the plasma density and magnetic field [Luhmann et a1., 1985]. Other 1ndirect evidence 1s that the position of the bow shock at the Venus terminator has been found to depend on solar activity. When the solar EUV flux is high it appears that more mass is being added to the shocked solar wind, forcing the bow shock to recede from the planet [Alexander and Russe11, 1985].

An 1nisresting asymmetry between the two draped lobes is observed In Figure 3.1. If this schematic is correct, the $X-$ component of the IMF has an Important 1mpact on the Internal magnetic configuration of the Venus magnetotail. As a consequence of the larger magnetic flux content of the tallward pointing lobe at all distances down the tall, the current sheet should be displaced to the right in an equilibrium configuration. As the IMF spiral angle (in the $X-Y$ plane) rotates, the current sheet should tend to flap from side to side within the tall in order to try to maintain an equilibrium condition. In this study we choose a coordinate system which preserves any asymmetries between the lobes, and examine the Importance of this effect in the morphology of the Venus magnetota11. 
The purpose of this paper is to examine the detalled average draping pattern of the magnetic fleld in the deep $\left(-8\right.$ to $\left.-12 R_{V}\right)$ Venus magnetota11. A detalled mapping of the average configuration of this region is made possible for the first time by a new technique. This technique removes the ambigulty of the spacecraft location with respect to the tall structures by utilizing an observed relationship between the magnetic fleld angle and the fleld magnitude referenced to the 1mmediately adjacent magnetosheath value, or diamagnetic reduction. A statistical study of the fleld variations with respect to the internal tall structure is thereby made possible, and the average plasma properties consistent with these variations are derived.

We will describe this study in sections. Section 3.2, "The Average Venus Tal1 in Magnetic Coordinates," examines the varlability of the data ordered by spatial location and lays the groundwork for developing a coordinate system which measures locations witi respect to the tall structures themselves. "The Average Tall Configuration with the Effects of Flapping Removed," 3.3, shows how we reconstruct the structure of the tall in the presence of flappling and examines the average varlations in the field components, culminating in the average field vectors, cross tal1 current density distribution, and $\underset{\sim}{\mathrm{B}} \times \mathbf{B}$ forces as functions of location across the tall. In Section 3.4, "Inferred Plasma Properties," we derive the average downtall velocity as a function of distance and define a simple model based on the fleld variations from which the average plasma acceleration as a function of 
distance, density, and temperature are obtained. Finally, Section 3.5 reviews most salient steps and results of our analysis.

\subsection{The Average Venus Ta1l in Magnetic Coordinates}

The data set used in this study is a large subset of the data set chosen by Saunders and Russell [1986]. It cons1sts of magnet1c fleld data from the Ploneer Venus orbiter (PVO) magnetometer [Russell et al., 1980b], and contains the magnetotall portions of 38 orblts which were selected from the first ten seasons of data (6/79-5/84). In a11, 9423 one-minute averaged magnetic field measurements are used. The criteria used to identify magnetotall portions of the data are variations in field strength and orlentation, and changes in the spectrum of fleld varlations; they are described in greater deta11, with examples, by Saunders and Russell [1986]. While the identification of these regions is not perfect, it is correct for the vast majority of the data, and the mixing in' of small portions of magnetosheath data will not substantially affect the statistical results described here.

The coordinate system used in this section of our study will be called the B-v coordinate system. The term B-v is used to Indicate that the orfentation of the coordinate system is determined by the average upstream magnetic field and solar wind flow directions. In the $B-v$ coordinate system the solar wind flows parallel to the $-X-$ direction, and the magnetic fleld component perpendicular to the

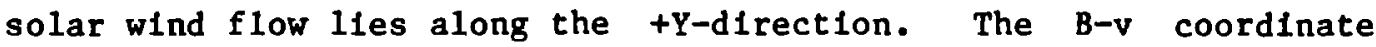
system is derived from the aberrated vSO coordinate system, 
described by Saunders and Russe11 [1986]. The VSO coordinate system is similar to the GSE coordinate system, except that it is centered on Venus and its pole is parallel to the Venus orbital pole. The VSO $X$ - and $Y$-axes are then rotated by $5^{\circ}$ about the $Z$-axis to remove the average aberration of the solar wind flow due to Venus ${ }^{-}$orbital motion.

In order to convert from an aberrated VSO coordinate system to a magnet1c fleld ordired system we have two cholces. The standard cholce would be to rotate about the solar wind direction so that the magnetic fleld projected on the $Y-Z$ plane always pointed in the same direction, e.g., +Y (Bleber and Stone [1979]; Saunders and Russe11 [1986]). There is a difflculty with this method, however, for if there is an asymmetry between the two lobes in the tall, as discussed previously, this method would average out the difference by combining instances of each type of lobe on both the $\pm Y$ sides of the tall. The alternative cholce is to assume that the sector polarity of the magnetic field is inconsequential to the draping of the fleld, and change the sense of each field line so that it corresponds to a magnetic field line pointing away from the sun. This gives a coordinate system where the fleld is always rotated by less than $\pm 90^{\circ}$ to project along the $+Y$-direction. This cholce permits the separation of "east-west" asymmetries in which the two lobes, parallel and antlparallel to the IMF, are different. However, it does not allow the detection of north-south differences as might occur, for example, if mass loading is asymetric as predfcted by Clout1er et al. [1974], [1976], and as is suggested by 
the findings of Saunders and Russell [1986] and Slavin et al. [1985]. There is no cholce of coordinate system that will allow us to determine simultaneously both "east-west" and "northsouth" asymmetries while combining data obtained in different solar wind sectors, since the interaction is inherently three-dimensional. However, in order to Increase our statistical accuracy we wish to combine such data. As will become evident in this study, the east-west asymmetry will be very Important and, therefore, we choose to convert the IMF polarities prior to rotating about the solar wind flow.

The IMF is highly variable and when the Ploneer Venus spacecraft is probing the Venus tall it spends little time in the solar wind. When avallable, any solar wind observations are far removed in time from the perlods of the tall observations. Ideally, It would be desirable to have a measure of the IMF direction when Ploneer Venus is within the tall. Saunciers and Russell [1986] have established that the cross tall or Y-component is everywhere, on average, parallel to the component of the upstream IMF that is perpendicular to the flow. This is what is expected if there is little reconnection of the fleld between the two tall lobes. Given these results we can use the direction of the cross tall component measured on each crossing through the tall to rotate Into the desired B-v coordinate system. During each tail crossing, the component of the fleld in the plane perpendicular to $X$ is measured, and 1 ts average direction is used to rotate the coordinate system 
for the entire crossing so that the average field profection lies along the $B-v+Y-d i$ rection.

Figure 3.2 shows the orbital coverage of our data set projected on to the $Y-Z$ plane of the the $B-V$ courdinate system. All data have been accepted in the full width of observation in the cross-flow, $Y$, direction; however, the data set has been truncated In the 2direction at \pm 1.6 Venus radi1 $\left(R_{V}\right)$. This truncation ensures that the draping, which is expected to occur somewhat differently as a function of $Z$ in the tall, due to the variation in the tall width with $\mathrm{Z}$, does not $\mathrm{mIx}$ together the draping patterns from very different portions of the tall. By choosing only the central portion where the tall width is falrly constant, and over which draping can be expected to be falrly similar, and then by compressing this data set Into the two-dimensional X-Y plane, we maximize the statistical accuracy of our ierivation of the twodimensional draping pattern in the tall. Further, our cholce of coordinate system has mixed together "north-south" asymetries so that our analysis has already suppressed the vertical gradients in the tall.

Small sections of orbits observed in this figure correspond to portions of the time when the orbit, although continuous in real space, is not continuous in the effective space of the $B-v$ coordinate system. These correspond to times when the IMF variation is large enough that the fleld points in a reverse cross-flow sense, as will be described shortly. At these times it is necessary to reflect not only the fleld, but also the location in the tail in the 


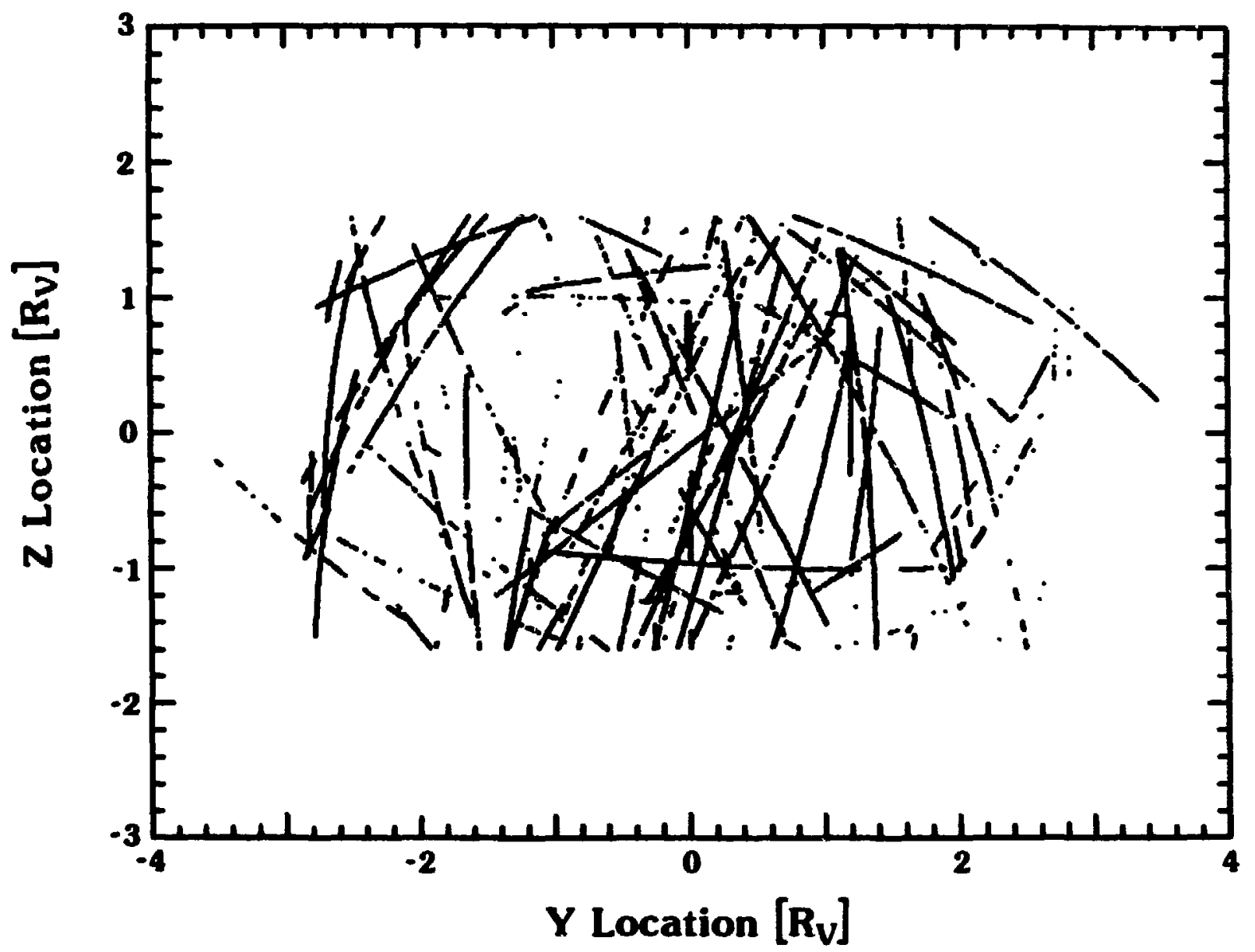

Fig. 3.2. The orbital coverage of our data set which includes 9423 one-minute averaged magnetic field data points. The data are shown in the $Y-Z$ plane of the cross-flow (B-v) coordinate system in which the upstream magnetic field points along the $+Y$ axis. The data set is truncated at $Z= \pm 1.6 R_{V}$ in order to reduce the effects of a circular tail cross section when the data set is compressed in $Z$. 
cross-flow coordinates so that toward and away sectors are, again, not mixed.

In both panels of Figure 3.3 the magnitude of the magnetic fleld in the tall divided by the average magnitude of the magnetic Field in the magnetosheath, lmmediately adjacent to the tall where the PVo plerces the magnetopause, on an orbit-by-orbit basis is plotted versus the angle of the fleld from the cross-flow $Y-Z$ direction toward the positive aberrated $X$-axis. of course, the magnitude of the magnetic field within the sheath is a function of location as well as the upstream conditions. Along the flanks of the tall from -8 to $-12 R_{V}$, however, the fractional magnetic fleld strength (compared to upstream) is probably not a strong function of the exact location of the PVO's magnetopause crossings and only serves to Introduce scatter Into the effect observed in Figure 3.3. St111, this figure clearly reveals a strong correlation between fields which are close to $\pm 90^{\circ}$ and the larger relative field strengths. We Identify these reglons where the fleld is comparatively strong and polnts basically tallward or Venusward as being the Induced lobes of the Venus magnetota1l. The term "Induced lobes" is used to distinguish these regions from the tall lobes in the terrestrial magnetosphere where the earth's intrinsic dipole fleld plays a fundamental role. At Venus the draped lobe regions are comprised purely of "hung up" Interplanetary magnetic field 11nes.

In contrast to the Induced lobes, the magnetic field in the Venus tall current sheet is observed to occur at small angles and at 


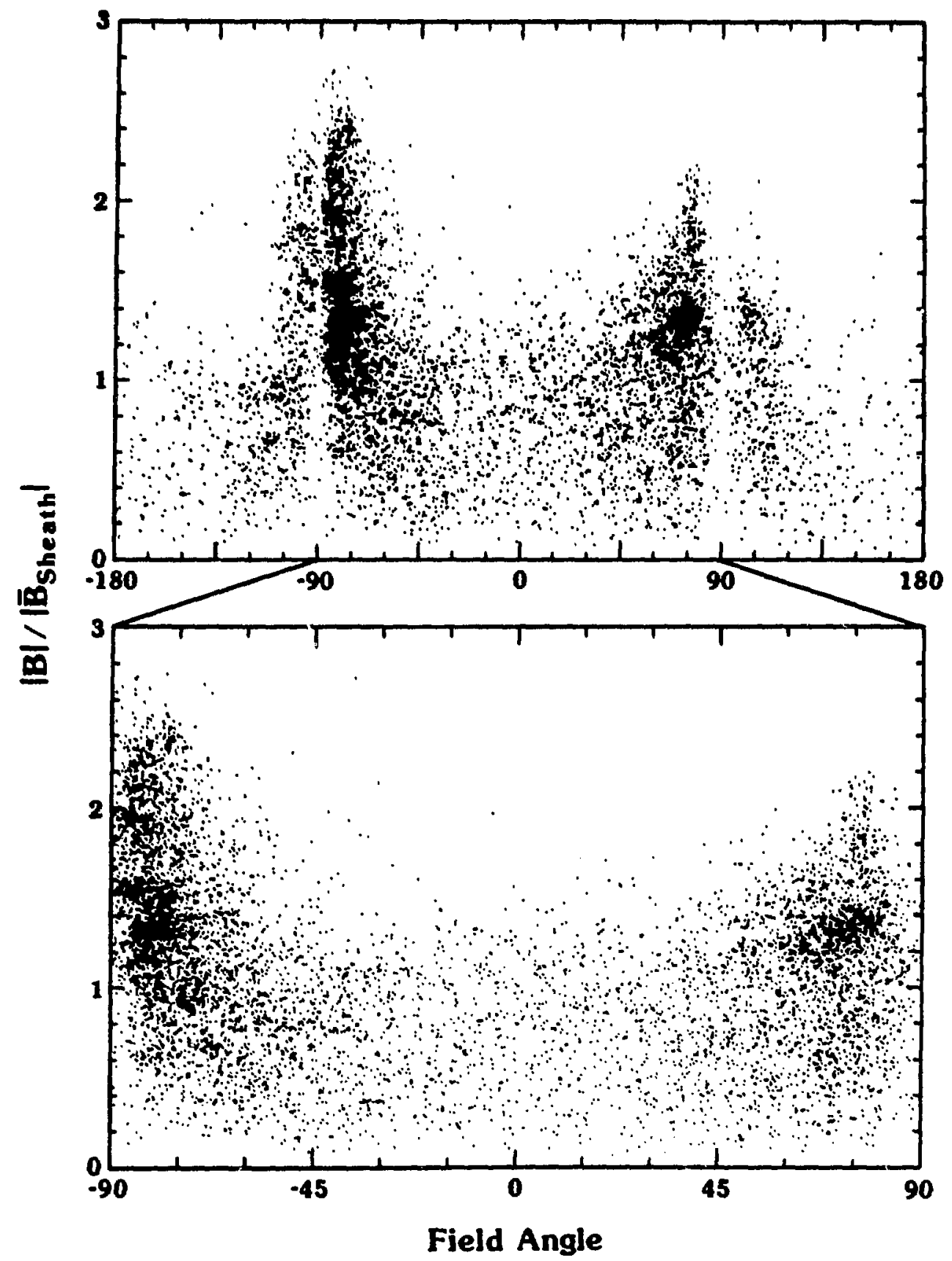

Fig. 3.3. The magnitude of the magnetic field divided by its average value in she immediately adjacent portion of the magnetosheath, plotted versus the magnetic field angle as measured from the crossflow $Y-Z$ direction toward the aberrated $X$ axis. The top panel shows a correlation between large relative fleld strengths and angles near $\pm 90^{\circ}$, and indicates that $79 \%$ of the data lie within these angles. The bottom panel shows the same data set where the remaining $21 \%$ of the dara have been folded into the central portion (see text). 
small field strengths. The decrease in the relative field strength may be due to a diamagnetic reduction in the center of the tail consistent with an Increase in the plasma density, as is observed in the earth's magnetotal1. Exclusions near $\pm 90^{\circ}$ may be due to the purely geometric effect of reduced solld angles near $\pm 90^{\circ}$, or may Indicate that the angles in the lobes never truly achieve $\pm 90^{\circ}$ and that the variations in aberration of the tail are smaller than or comparable to the spread in the draping angle in these lobes.

Twenty-one percent of the data in the top panel of the figure are observed to lie outside of $\pm 90^{\circ}$. In order to deternine whether this was a property of the tall, or caused by the variability of the upstream conditions, the same analysis which ylelded this result was repeated on 2701 minutes of magnetosheath data taken fust outside the Venus tall over the same orbits as in our tall data set. In the magnetosheath this reverse draping, which is indicated by angles outside of $\pm 90^{\circ}$, occurred $16 \%$ of the time. Therefore, approximately $16 \%$ out of $21 \%$ of the tall data which lies outside of $\pm 90^{\circ}$ is accounted for simply by the fluctuations in the upstream IMF direction causirg reverse draping. The extra $5 \%$ which is still unaccounted for may be due to reconnection or other phystcal processes. In any case, for the first time, an upper bound has been set on the Importance of magnetic reconnection in the Venus magnetota11. Only $\sim 5 \%$ of the observed field in the Venus magnetotall points in a reverse draped sense, and therefore our analysis indicates that magnetic reconnection is probably not an Important physical process in the central Venus magnetota11. 
The same data set is shown in the bottom panel of Figure 3.3 , with the measurements outside of $\pm 90^{\circ}$ "folded" across the $\pm 90^{\circ}$ 11nes. This folded data set w111 be used throughout the remainder of this study in $o_{2}$ der to obtain the most statistically significant results. Folding is reasonable since the observed variation between lobe and current sheet also occurs outside of $\pm 90^{\circ}$ where the $0^{\circ}$ current sheet area is similar to the $\pm 180^{\circ}$ portion. Since $3 / 4$ of the data outside of $\pm 90^{\circ}$ can be accounted for by variations in the IMF, truncation of these data would principally lower the statistics of the data set. It is therefore advantageous to fold these data back into the data set by reflecting the data points across $\pm 90^{\circ}$.

A similar plot for orbit 1761 alone is shown in Figure 3.4 . (The magnetometer data for thts orbit are displayed in Fig. 2 of Saunders and Russell [1986].) The data points have been connected to show the time series in which the data were taken. In motions between the two lobes, the spacecraft always encounters the current shert. That is, there are one or more data points obtained within the current sheet, which are characterized by sma11 angles and small fleld strengths, in the transition from one lobe to another. The motion of the spacecraft back and forth in the taill is clearly not due to the spacecraft orbital motion ( 24 hours/orbit), since there are many of these comparatively quick (a few minutes) crossings in a single orbit. Rather, some sort of large variations in the tail configuration (perhaps due to the IMF $X$-component) and/or in its location occurs on the time scale of a few one-minute data samples. These variations cause the spacecraft to be alternately in one lobe 


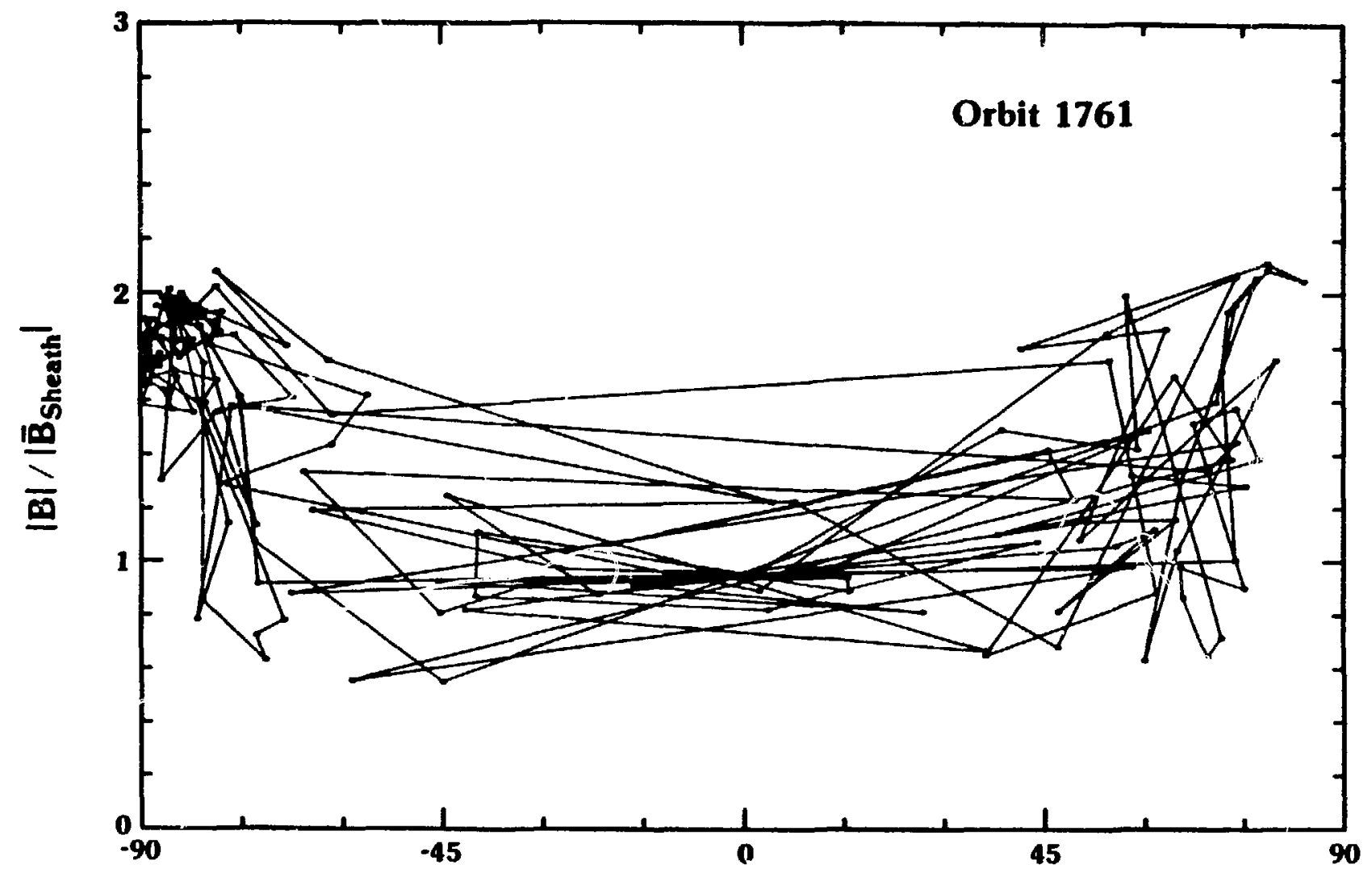

Field Angle

Fig. 3.4. Similar to Figure 3.3 except that only data from orbit 1761 are shown. The data points have been connected to show the time series in which the data were taken. The spacecraft traverses the tail many times in an individual orbit. Evidently the size scales of motions and/or reconfigurations of the tail are much larger than those due to the actual orbital motion. Therefore, the data will be much better ordered by a coordinate system which measures locations with respect to the internal tail structures rather than one which measures them in normal spacecraft coordinates. 
and then the other, crossing back and forth across the current sheet many times. This temporal varlation has previously made it impossible to do a thorough analysis of the average spatial structures of the Venus tall. These large vartations, however, are used in a new way in the next section of this study to 1mprove the statistics.

The fleld angle versus the $B-v \quad Y$ location 1 s shown in Figure 3.5. Current sheet(s) and lobes are observed at all locations where the tall is encountered, which is consistent with the observations of large variations in most orbits, and as is exemplified by the plot of orbit 1761. In Figure 3.5 there is a clear preponderance of either tallward or Venusward pointing fields on each of the two sides of the tail. Magnetic flelds near $+90^{\circ}$ occur preferentially on the $-Y$ side of the tall, while fields oriented near $-90^{\circ}$ occur predominantly on the $+Y$ side. In Figure 3.6 this finding is quantifled.

For the purposes of determining the number of induced tall lobes, we have, somewhat arbitrarily, defined them as consisting of all data points in which 1) the field strength divided by the directly adjacent sheath value (as described previously) is greater than one, and 2) the absolute value of the angle is greater than $60^{\circ}$ (upper left and right corners of Fig. 3.4 and the lower panel of F1g. 3.3). The fraction of the tallward pointing tall lobe is plotted versus the $B-v \quad Y$ location in Figure 3.6, and two draped lobes are clearly indicated. The number of draped lobes was not something that was assumed in our analysis, but rather it is a 


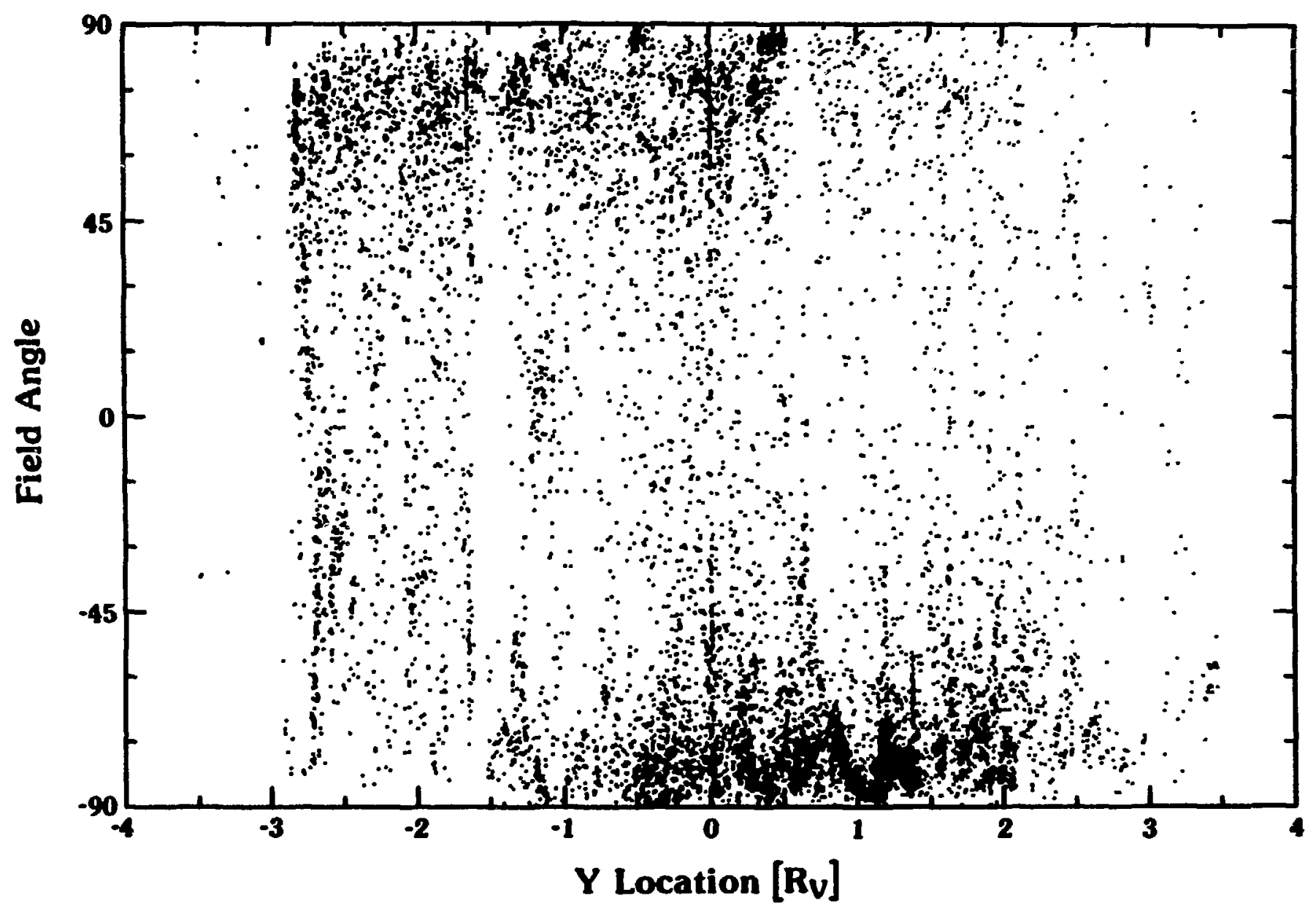

Fig. 3.5. The magnetic field draping angle versus the B-v Y location. Both direction lobes and current sheet(s) are observed at all locations antisunward of Venus. There is, however, more tailward pointing lobe data on one side of the plot and more Venusward pointing data on the other side. 


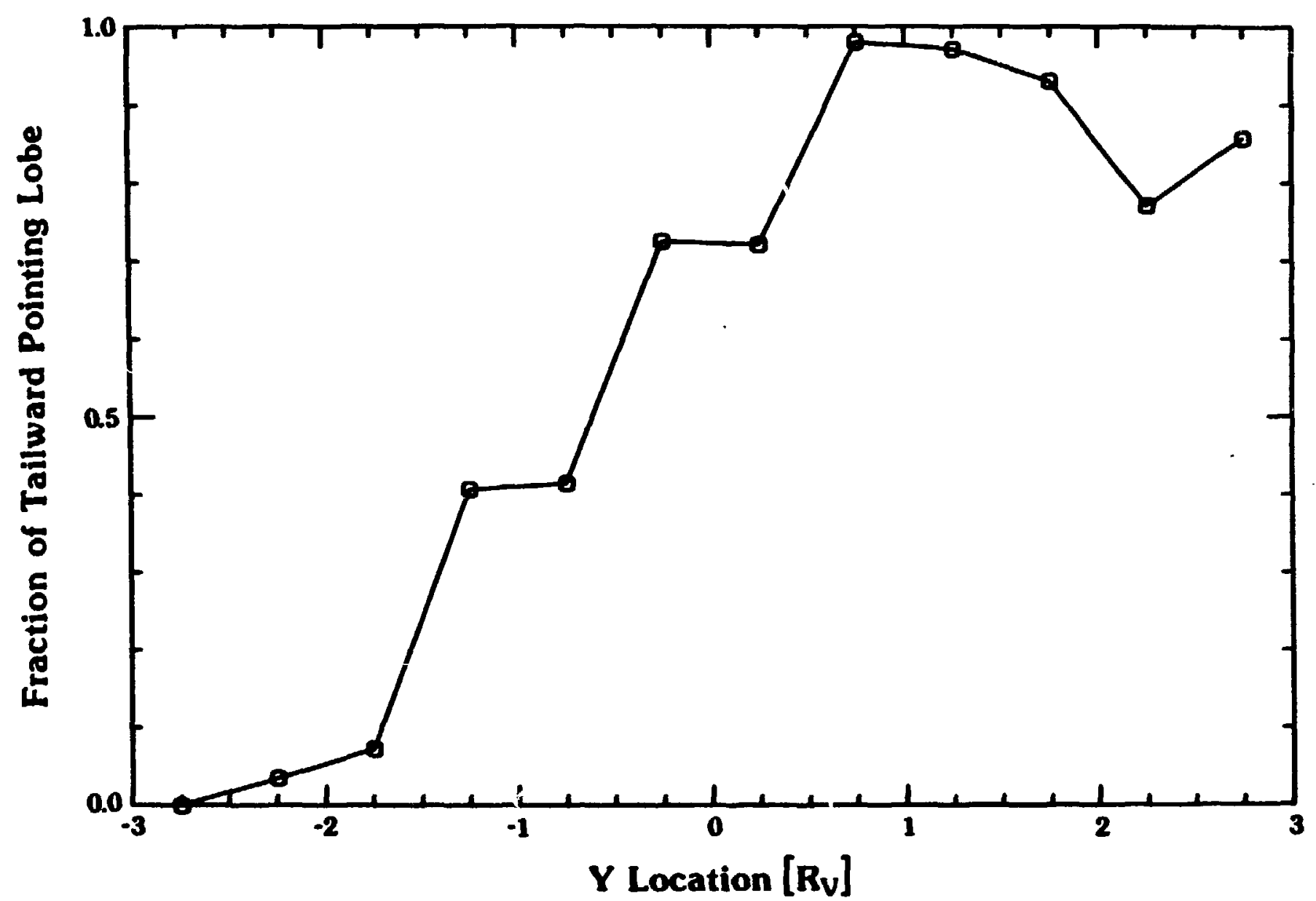

Fig. 3.6. The fraction of tailward pointing lobe as a function of the B-V Y location. The average tail configuration consists of only two draped lobes which are separated by a single field reversing current sheet. 
result confirmed by our analysis, which merely assumed that magnetic field draping is the important phenomena in the tall. We have repeated this analysis using other lobe cutoff criteria, and all yield the same result.

Figure 3.4 demonstrated that drape 1 lobes are separated by current sheets, wh1le Figure 3.6 conf1rms that there are only two lobes in the Venus magnetotail. Therefore, the Venus magnetotall structure is shown to consist of two roughly opposite pointing draped lobes separated by a single current sheet fust as we anticlpated in Figure 3.1. The average location of the current sheet center is approximately $-0.5 \mathrm{R}_{\mathrm{V}}$. This is consistent with the IMF X-component mapping into the tall such that, for a normal Parker spiral pattern and an away-sector, the magnetic fIux in the draped lobe which contains the tallward pointing fleld is larger than the flux in the draped lobe containing the Venusward pointing magnetic field. In a pressure balance situation, the current sheet between these two lobes will tend to move toward the side of the Venusward pointing lobe.

Since we are principally interested in determining the average configuration of the Venus magnetotall, the average field line draping angles in the lobes must be determined. Figure 3.7 shows the measured average lobe angles determined using the lobe criteria described above for Figure 3.6. Other, similar lobe criteria have also been tried and yleld very slmilar average angles. These fleld angles in the two lobes will be used to set the average angle to which the fleld drapes in the lobes on the distant sides of the 


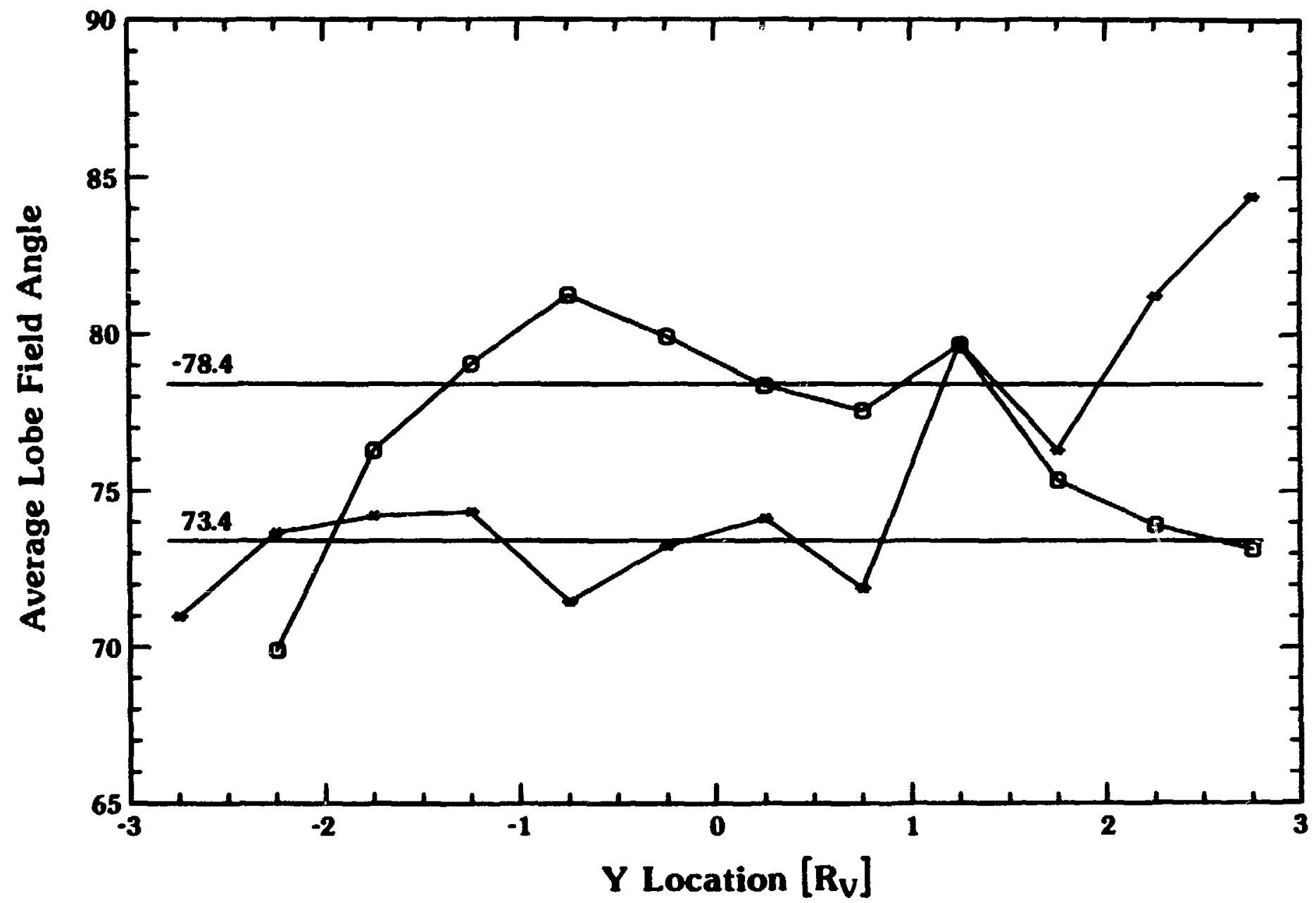

Fig. 3.7. The average angles of the field line draping in the outer portions of the two lobes. The tailward pointing lobe value is $-78.4^{\circ}$ while the Venusward pointing lobe is $+73.4^{\circ}$. 
ta11. The tallward polnting lobe average angle $1 \mathrm{~s}-78.4^{\circ}$, whlle the Venusward average angle is $+73.4^{\circ}$. This asymmetry can also be accounted for quite simply by the mapping of the IMF X-component through the magnetosheath and into the tall as shown in Figure 3.1.

3.3 The Average Ta11 Conflguration with the Effects of Flapping Removed

In all previous studies, and thus far in this study, the Venus magnetotall date have been ordered only by the spatial location of the PVO with respect to Venus. Our analysis, however, has indicated that motions and/or reconfigurations of the tall happen far more rapldiy than the PVO orbltal motion, causing the spacecraft to traverse the tall many times per orblt. In order to advance the understanding of the Venus magnetotall quantitatively, it is necessary to find a coordinate system which better orders these highly variable data by statistically organizing them with respect to the tall features themselves. Since a relation between the diamagnetic reduction and the fleld angle has already been demonstrated, it is reasonable to construct a coordinate system in which cross-flow locations do not correspond to locations in physical space, but rather correspond to locations with respect to the center of the moving or flapping current sheet.

For the data set used in this study, we have empirically determined the tall width to be $5.1 \mathrm{R}_{\mathrm{V}}$. This was accomplished by determining the fraction of time that each spatial location behind Venus was engulfed within the tall. Since tall motions are large, 
even the average center location of the tall was apparently within the actual tall only $\sim 97 \%$ of the time, and locations to the sides were within the tall far less. Under these conditions it is not appropriate to simply find the locations which are within the tail $50 \%$ of the time, and determine the average width of the tall from these. Rather, the fractional tall coverage behind the planet must be Integrated, and the effective tall width calculated from it such that this integrated value equals the tail width multiplied by $100 \%$ (of the time).

We have already shown that the magnetotall is comprised of two draped lobes separated by a cross tall current sheet and that fields on the $+Y$ side of the tall point tallward, while the fields on the -Y side point Venusward for an away IMF sector draping. Further, since the diamagnetic reduction apparentiy gives a measure of the average distance from the center of the current sheet, we can now build a coordinate system which is centered on the current sheet and which measures distances from that center. Large varlations in the tail configuration and location cause all regions to be sampled at any physical location in the tall. In a statistical data set, such as the one used for this study, the fraction of the total data points which lie within any given angular range gives a direct measura of the relative width of that portion of the tall. The sum of all of these relative widths must, of course, equal the total width of the Venus tail, $5.1 R_{V}$. It is then possible to construct the average configuration of the tall by starting with the average 
angles derived in each of the two lobes and calculating the thickness of each of the angular bins across the tall.

The basic principal involved in creating such a coordinate system is shown schematically in Figure 3.8. For simplicity the ta11 has been divided into only three portions, namely, the $+x$ pointing lobe, the $-X$ pointing lobe, and the current sheet. The lower portion of the figure is a schematic diagram of Figure 3.5. Since varfations of the spacecraft position with respect to the Internal structures of the tall are so large, all reglons are sampled at every B-v Y location, and the fraction of fleld in each of the three angle bins gives a direct measure of the thickness of that portion of the tall. In this example the $+X$ lobe, current sheet, and $-\mathrm{X}$ lobe are $32 \%, 28 \%$, and $40 \%$ of the tall width respectively. Since the tall is $5.1 \mathrm{R}_{\mathrm{V}}$ across these would correspond to $1.6,1.4$, and $2.1 \mathrm{R}_{\mathrm{V}}$ for each portion, respectively. This technique is simply extended in our study to measure the thickness of each one degrise angle bin across the tall, and thereby we reconstruct the average tall configuration. The resultant $Y$ * coordinate axis constructed by this technique is paralle1 to, and shares a Venus centered origin with, the $B-v Y$-axis.

The angle of the magnetic fleld versus this cross tall location, $Y^{*}$, is shown in Figure 3.9. The cross tall locations of varfous angles in the tafl have been determined as described in the previous figure while the $X$-axis in these new coordinates is the same as the X-axis in the aberrated VSO coordinates. The procedure described above of dividing up and determining the relative amount 

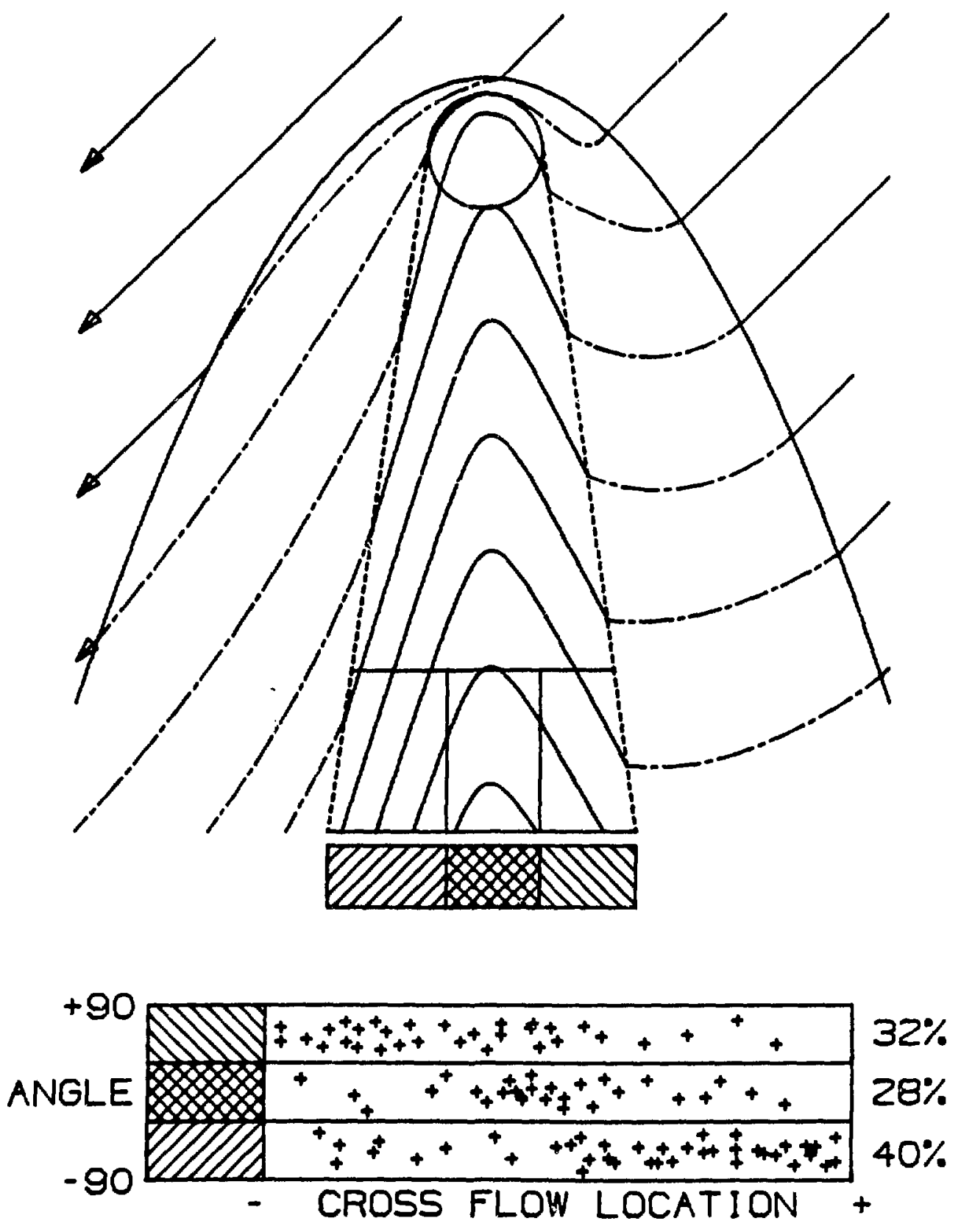

Fig. 3.8. Schematic diagram of the basic principle used in defining our constructed coordinate system which measures locations with respect to the internal tall structure. The fraction of data points within any range of angles gives the relative thickness of the portion of the tail which encompasses those angles. Combining this information with the total, physical width of the tail gives the spatial scale to the cross tall coordinate axis. 


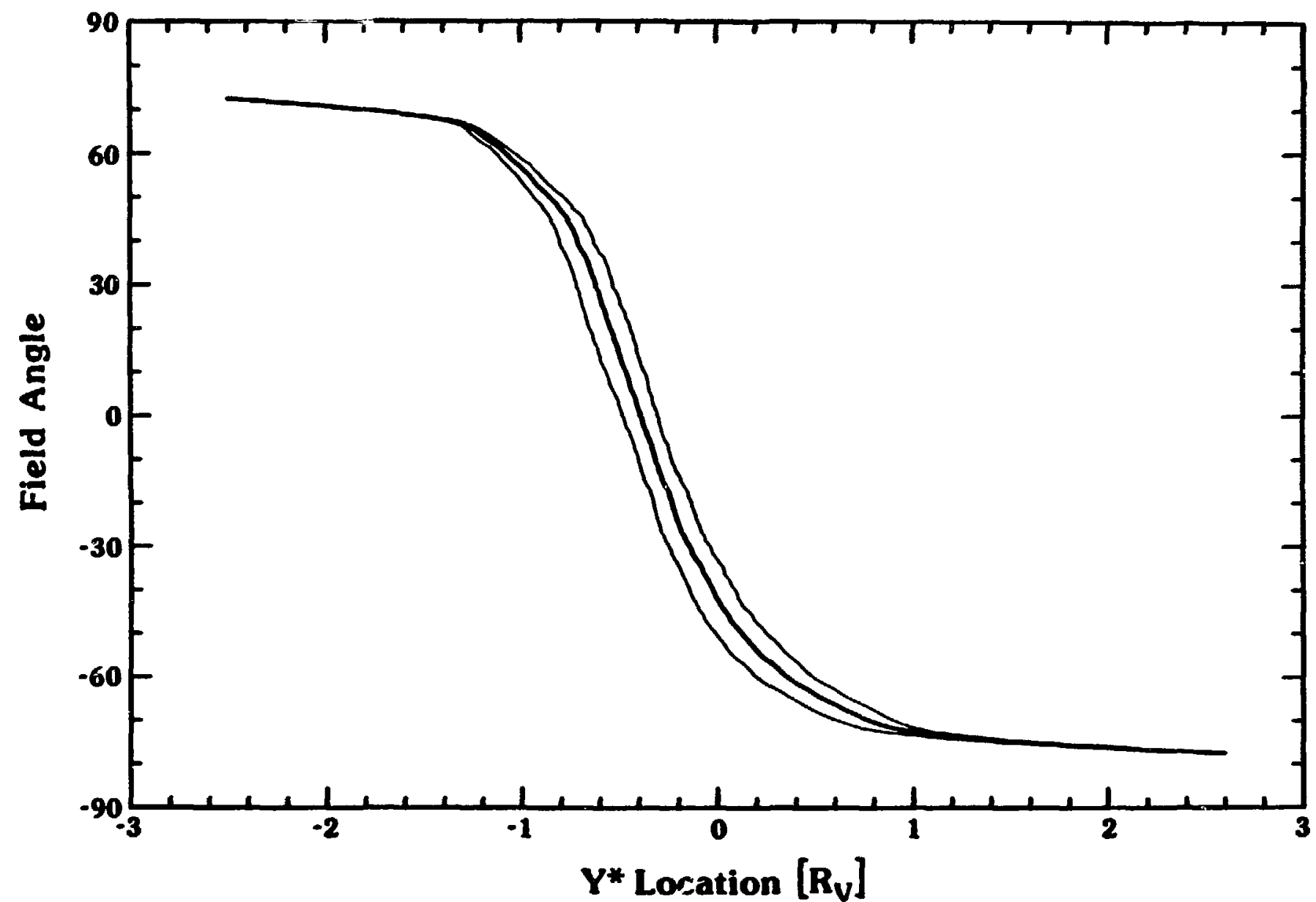

Fig. 3.9. The angle of the magnetic field plotted versus the $Y *$ location. The two lighter lines are the upper and lower bounds found by carrying out our analysis over $\pm 0.5,1.0,1.5,2.0 \mathrm{R}_{\mathrm{v}}$. The darker, center line is the average of these and is adopted for the remainder of this study. 
The tallward/Venusward magnet1c fleld, $B_{X}$, 1 s plotted versus $Y^{*}$ in Figure 3.10. These are actual measurements over the 9423 data points ifnned up angle by angle and compared to their $\mathrm{Y}^{*}$ locations, and are not calculated from the angles used to derive the (*) coordinates. This plot, therefore, gives a good independent check of the ability of the $(*)$ coordinate system to order the data. The two draped lobes are easily discerned in this plot as is the smooth variation between the two through the current sheet. The zero point in $B_{X}$ once again occurs slightly offset toward the negative slde of the tall. The variation displayed has been calculated in an average sense over $X$; the entire data set has been compressed In $X$ so that only variations in the" cross tall direction are determined. Throughout this paper such averages w111 be indicated with $\langle$, so that ${ }^{B} \mathrm{X}$ as a function of $Y^{*}$, and averaged over $X$ is written $\left\langle\mathrm{B}_{\mathrm{X}}\left(\mathrm{Y}^{*}\right)\right\rangle_{\mathrm{X}}$. Th1s average $\mathrm{B}_{\mathrm{X}}$ as a function of $\mathrm{Y}^{*}$ will be used shortly to calculate one of the terms of the current density distribution in the cross tail current sheet.

A similar plot for the cross-flow inagnetic field, $B_{Y^{*}}$ as a function of $\mathrm{Y}^{*}$ is displayed in Figure 3.11. The distribution has been calculated in 11 bins in $Y^{*}$ to fmprove the statistics. The double humped variation exhibited in this plot is qualitatively similar to the variation observed in the Fedder simulation of cometary magnetotalls (J. Phillips, private communication; Fedder et a1. [1986]). By combining the results in Figures 3.10 and 3.1i, the field draping in an average sense across the tall is determined. This average draping pattern is shown in Figure 3.12. It must be 
of data in each one degree angle bin has been carried out over \pm 0.5 , 1.0, 1.5, and $2.0 R_{V}$ in $B-v Y$ from Figure 3.5. A11 four of these analyses were carried out independently, and the minimum and maximum angles of the four have been plotted at each $\mathrm{Y}^{*}$ location to give the upper and lower bounds, displayed as the two light lines in Figure 3.9. The darker, center line is the average of these four analyses and is adopted for the purposes of this study hereafter. The very small varlation between the center average line and the minimum and maximum lines indicates that it is relatively unimportant over how much of the central portion of the tail the analysis is done. Variations are sufficiently large in tail configuration and location that all portions of the tail are sampled with little orbital prejudice everywhere between \pm 0.5 and $\pm 2.0 R_{V}$. This, then, is the (*) coordinate system in which we can much better measure the average physical features of the Venus tall.

Angles beyond the average angles found in the lobes have been folded into the angles just below the average lobe angles. This is necessary because the analysis fails as the lobe draping angle approaches $\pm 90^{\circ}$, and at angles of $\pm 90^{\circ}$, the field 1ines would apparentiy not cross the tall at a11. We expect that these infrequently measured large angles are not characteristic of the actual, averaged draping, and probably result from variations in the aberration angle of the tail. Since we are principally interested In the average magnetic fleld draping in the tail, it is desirable to have the fields come to their average lobe values at the edges of the lobes. 
The tallward/Venusward magnetic field, $B_{X}$, is plotted versus $Y^{*}$ In Figure 3.10. These are actual measurements over the 9423 data polnts blnned up angle by angle and compared to their Y* locations, and are not calculated from the angles used to derfve the (*) coordinates. This plot, therefore, gives a good Independent check of the ability of the (*) coordinate system to order the data. The two draped lobes are easily discerned In this plot as is the smooth varlation between the two through the current sheet. The zero point In $B_{X}$ once again occurs slightly offset toward the negative side of the tail. The variation displayed has been calculated in an average sense over $X$; the entIre data set has been compressed $1 n X$ so that only varlations in the cross tall direction are determined. Throughout this paper such averages w111 be Indicated with $\langle$, so that ${ }^{B} X$ as a function of $Y^{\star}$, and averaged over $X$ is written $\left\langle\mathrm{B}_{\mathrm{X}}\left(\mathrm{Y}^{*}\right)\right\rangle_{\mathrm{X}}$. This average $\mathrm{B}_{\mathrm{X}}$ as a function of $\mathrm{Y}^{\star}$ will be used shortly to calculate one of the terms of the current density distribution in the cross tall current sheet.

A simflar plot for the cross-flow magnetic fleld, $B_{Y} *$ as a function of $\mathrm{Y}^{\star}$ is displayed In Figure 3.11. The distribution has been calculated in 11 bins in $Y^{*}$ to improve the statistics. The double humped variation exhibited in this plot is qualitatively similar to the variation observed in the Fedder simulation of cometary magnetotalls (J. Phillips, private communicatior; Fedder et a1. [1986]). By combining the results In Figures 3.10 and 3.11 , the fleld draping in an average sense across the tal1 is determined. This average draping pattern is shown in Figure 3.12. It must be 


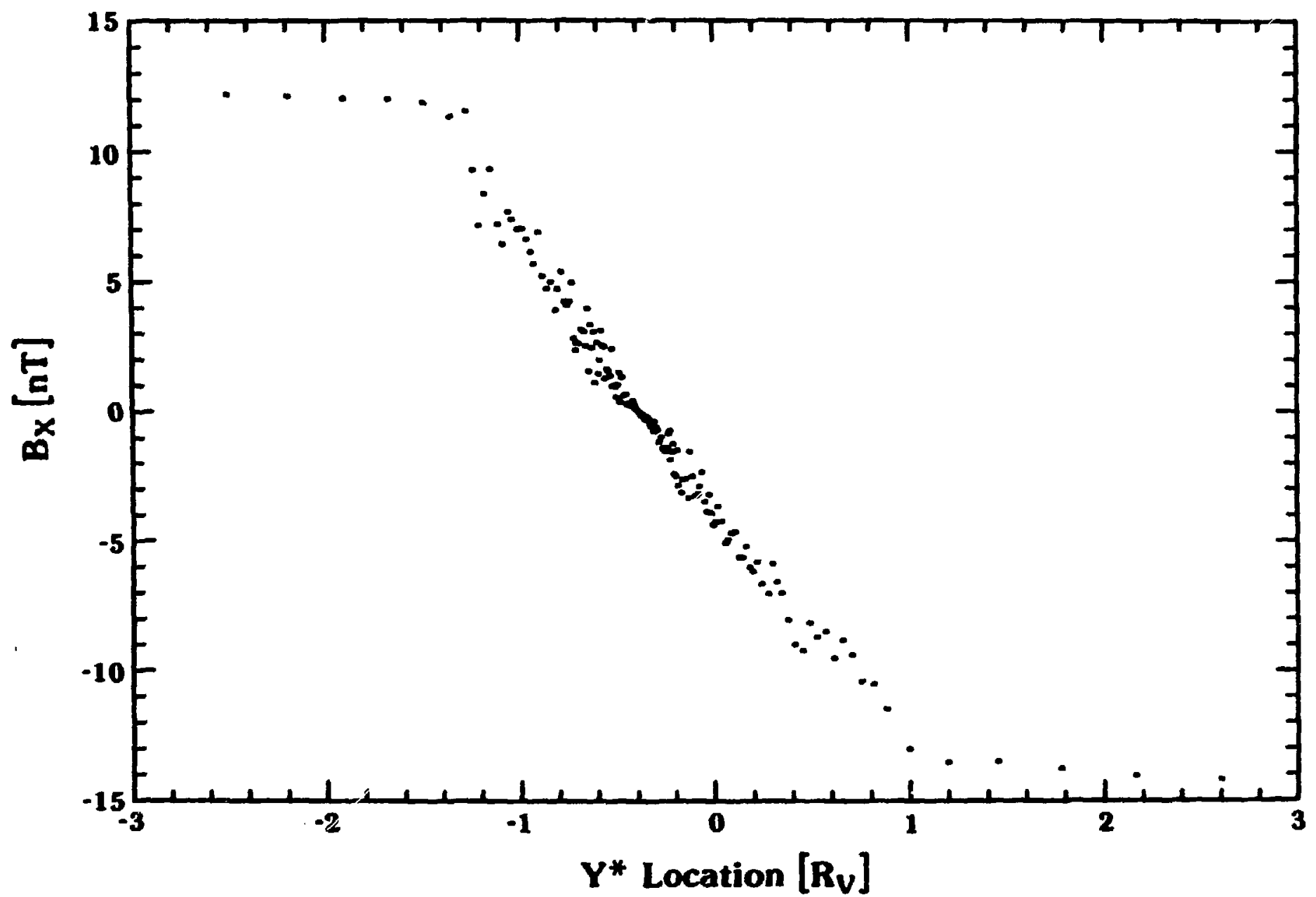

Fig. 3.10. The actual, measured $B_{Y}$ plotted versus $Y *$ in each of the one degree angle bins. This plot gives a good independent check of the ability of the coordinate system to order the data set. 


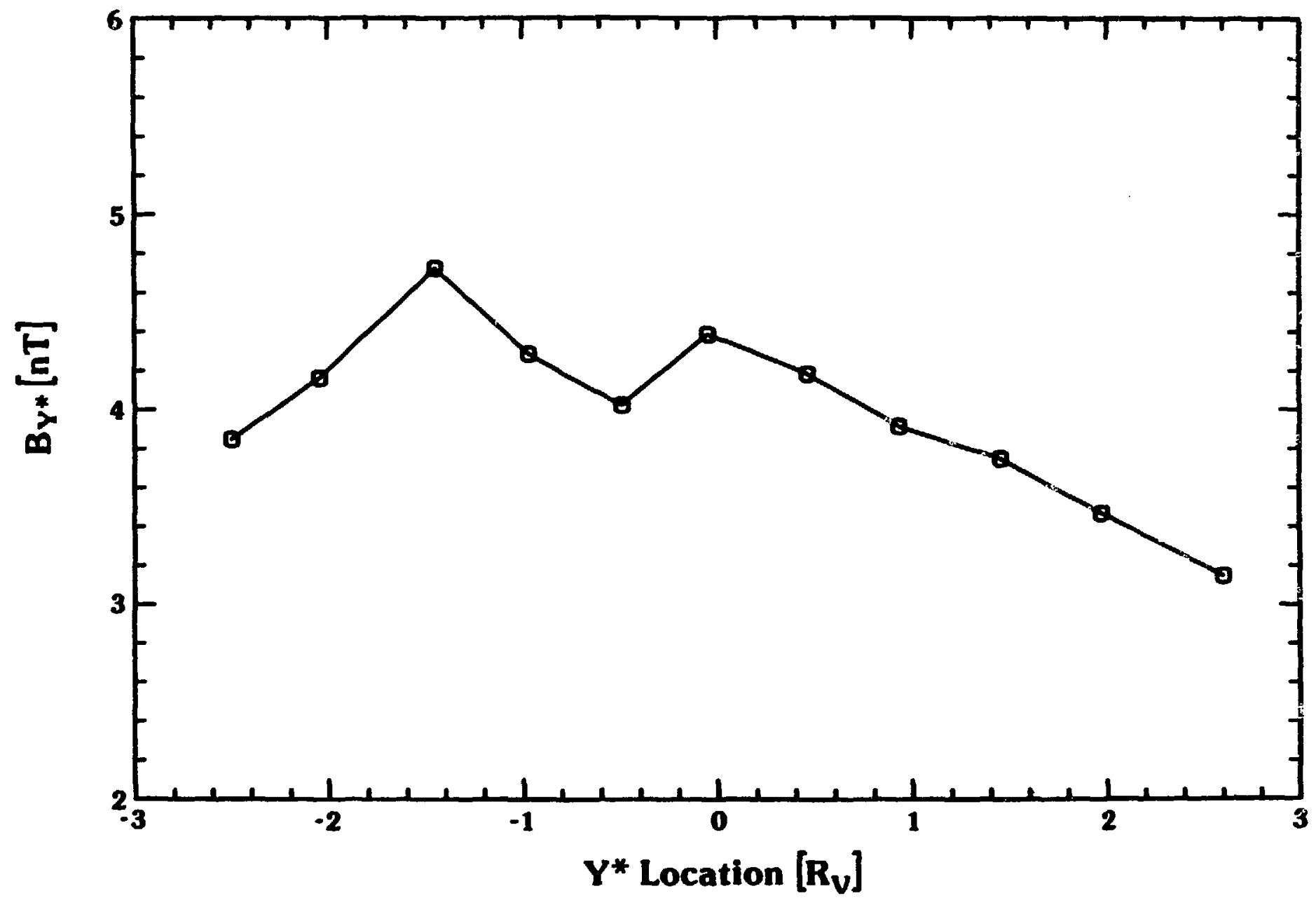

Fig. 3.11. The measured $B_{Y *}$ plotted versus $Y *$. The variation is calculated in eleven bins acrcss. the tail, which vastly improves the statistics. 


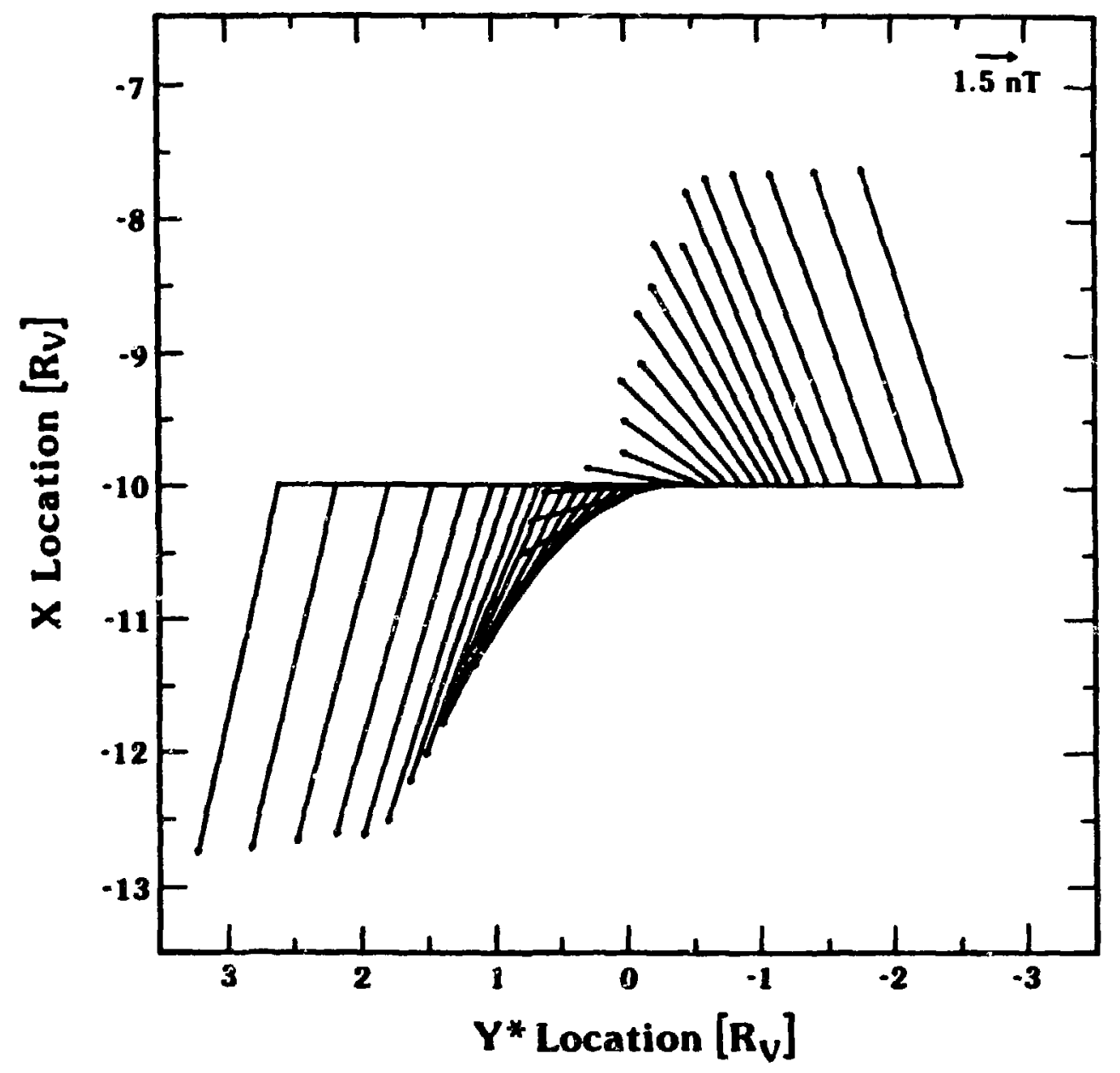

Fig. 3.12. The average magnetic field vectors determined with our technique as a function of the Y* location across the tail. The two draped lobes and the smooth rotation through the current sheet between them are all clearly shown. 
emphastzed that these are not magnetlc fleld lines, but rather average field vectors in a crosscut through the tall along $\mathrm{Y}^{*}$. Data $f=0 m-8$ to -12 Venus rad11 have been compressed in $X$ to glve this draping pattern. Figure 3.12 clearly displays the two draped lobes and current sheet in which the fleld smoothly rotates from one lobe to the other. The tallward side is thicker due to larger flux content, as well as having a larger tallward fleld component due to the mapping in of the X-component of the IMF, just as we postulated In Figure 3.1.

Next, we extend the understanding of the fleld draping in the tail by examining varlations of the field in the X-direction. Unfortunately, it is only possible to examine the $\mathrm{B}_{\mathrm{Y}^{*}}$ fleld varlation with $X$ since the $B_{X}$ variation is such a critical function of sampling location in $\mathrm{Y}^{*} \cdot \mathrm{B}_{\mathrm{X}}$ varles from about \pm 1.3 in in the lobes to zero in the center of the current sheet, and, therefore, any orbital bias can strongly affect the determination of the exact varlation of the $\mathrm{X}$-component. On the other hand, the $\mathrm{B}_{\mathrm{Y}^{*}}$-componert is relatively constant across the tall and is therefore not much affected by orbital blas. In the top panel of Figure 3.13 we show the orbital coverage in $0.5 R_{V}$ bins, where data from -6 to $-12 R_{V}$ have been included in order to maximize the region cr, sered. The distribution strongly favors data belng taken past $-10 R_{V}$ due to the fact that the PVO orbit apoaps1s occurs near $-12 R_{V}$.

The lower panel of Flgure 3.13 displays $B_{Y^{*}}(X)$ along with the best linear fit to this varlation. This best fit line equally weights all data polnts and does not, therefore, overly stress the 

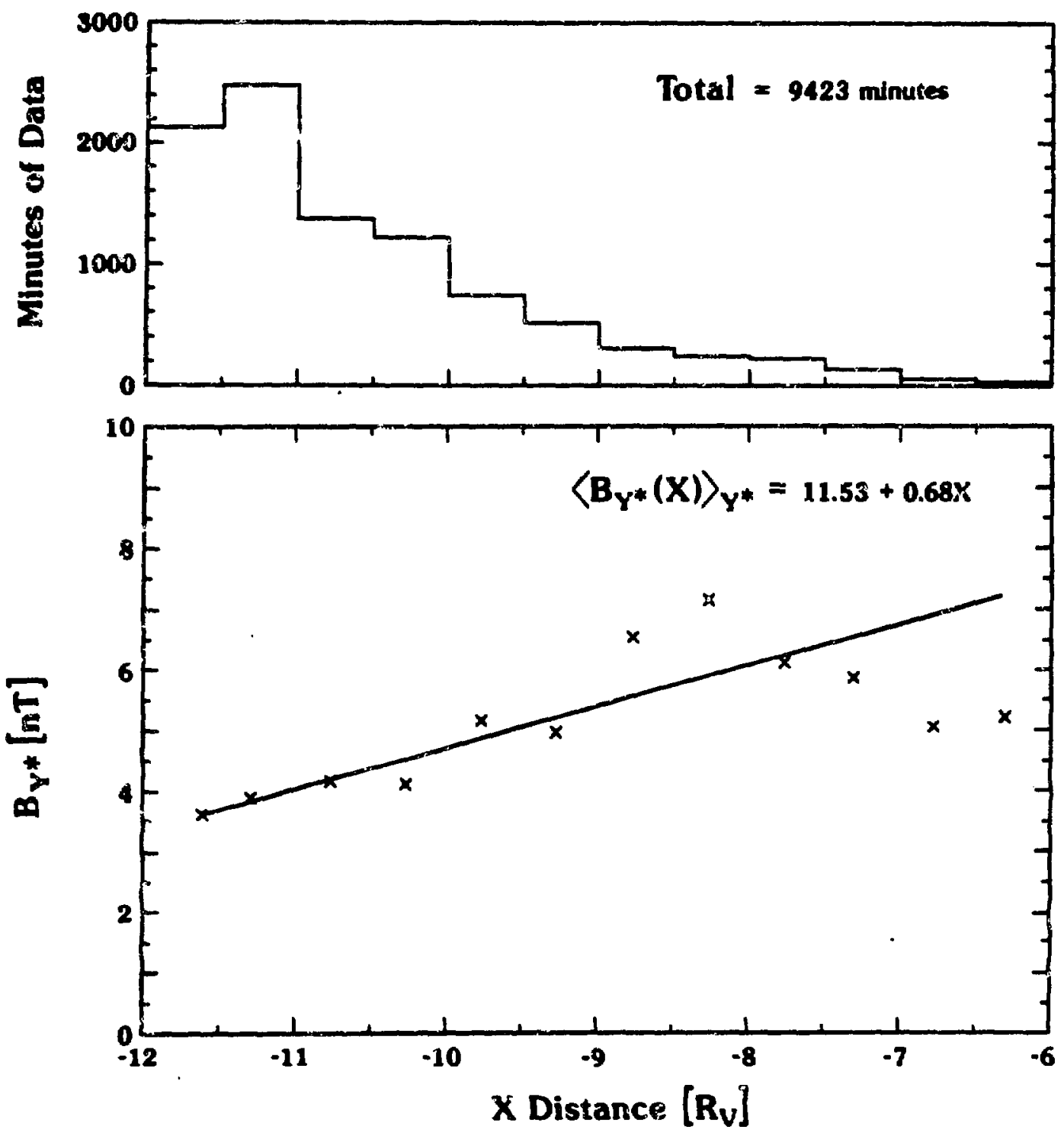

Fig. 3.13. The previous analysis is extended to examine the variation of the field with downtail, $X$, distance. The top panel shows the orbital coverage of our data set as a function of $X$, while the boicom panel shows the $Y^{*}$-averaged variation of $B_{Y *}(X)$. The best linear fit to this variation is also shown. 
less signiflcant data blng closer to the planet. The equation for this begt fit line for $B_{Y *}$ as a function of $X$, again averaged over $Y^{*}, 1 \mathrm{~s}\left\langle B_{Y^{*}}(X)\right\rangle_{Y^{*}}=11.53+.68(X)$ in $\mathrm{nT}$, where $X$ is in Venus rad11. From this variacion and from the variation which was shown in Figure 3.10 , the cross tall cuirent density can be calculated.

The cross tall current density is numerteally evaluated from the curl of the magnetic fleld according to Ampere's Law. Using the

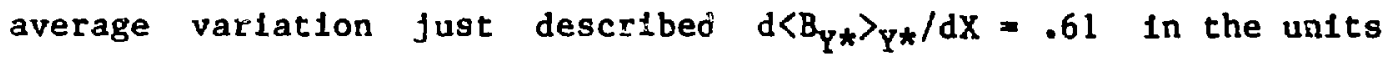
of $n T / R_{V}$ from the linear fit to the fleld variation. This gives a sma11 offset most easily observed in Figure 3.14 at the two far sidf: of the tall, while the principal variation in current density through the center is due to the term $d B_{X} / d Y^{*}$ and 1 s calculated from the variation shown in Figure 3.10. For Figure 3.14 we have derived the c.oss tall current density in 11 steps in $Y *$ to smooth out variations which are observed in the derivatives. Clearly the cross tall current density is maximized near the center of the tall at $-.5 R_{V}$ and drops to very sma11. values which correspond primarily to the linear $\mathrm{dB}_{\mathrm{Y}^{*}} / \mathrm{dX}$ term near the edges of the lobes.

From this calculated variation and from the field which was shown in FIgure 3.12, the $\underset{\sim}{\mathrm{J}} \mathrm{B}$ forces can be calculated in an average sense across the tail. These forces are shown In Figure 3.15. All forces have a tallward component, and these - $\mathrm{X}$-directed forces maximize in the center. Forces on both sides of the tail all point toward the center of the tail. Throughout the side portions of the tall these Inward forces, $F_{Y}$, are greater than tallward $F_{X}$ forces. Asymmetries in the side to side distribution of forces are due not 


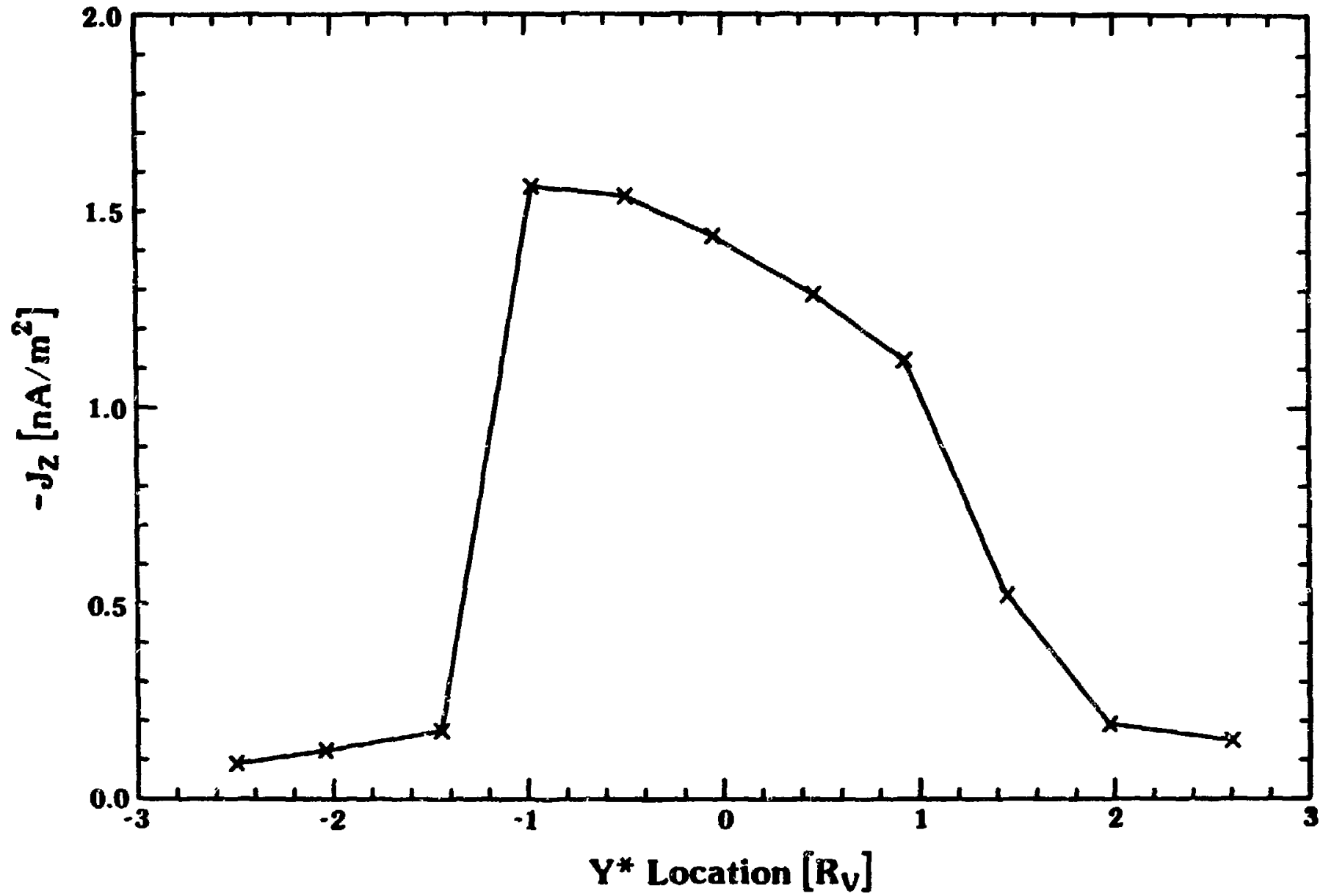

Fig. 3.14. The cross tall current density as a function of cross tail location. Both terms of the curl of the field are included in this calculation. $\partial B_{\mathrm{Y}} / \partial \mathrm{X}$ gives a small constant iffset which is most easily observed at the two edges of the tail, while the primary component through the center of the tail is from $\partial B_{X} / \partial Y^{*}$. 


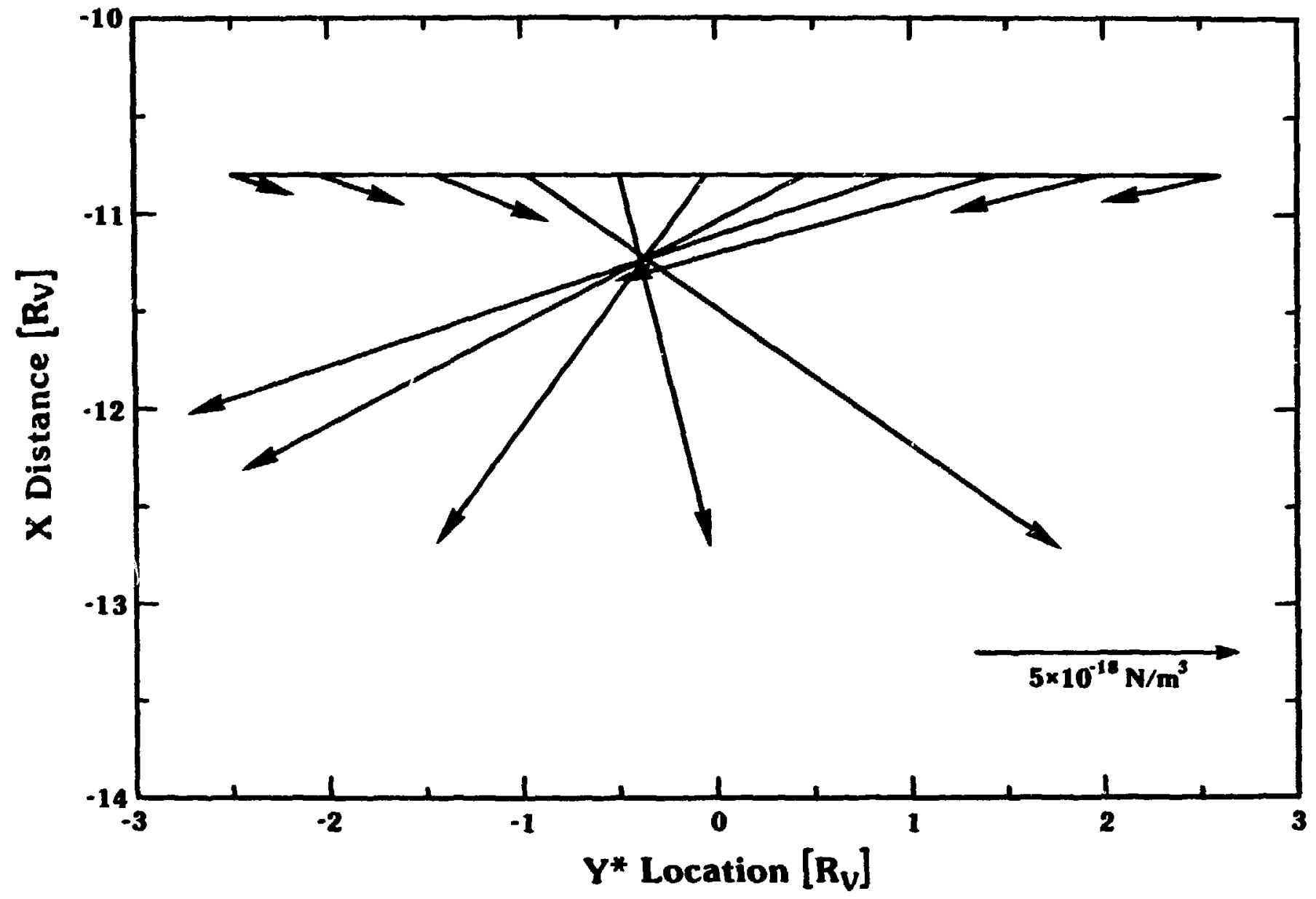

Fig. 3.15. The derived $\mathrm{J} X \mathrm{~B}$ forces as a function of $\mathrm{Y} *$ across the tail. The $\mathrm{X}$ directed forces all point tailward and maximize in the tail center, while the $Y *$ directed forces point inward on both sides. These forces are the important electromagnetic term of the momentum equation with which we examine the consistent plasma properties of the Venus magnetotail. 
only to actual asymmetries in the tall configuration, but also, to some extent, to the coarseness of our sampling in $Y^{*}$.

\subsection{The Inferred Plasma Propertles}

The average varlations of the magnetic fleld draping in the Venus magnetotall are not just interesting in their own right, but also contain significant information about the average plasma properties throughout this region. In particula., the $\mathrm{X}$ variations of the downtail plasma velocity, $v_{X}(X)$, and acceleration, $a_{X}(X)$, can be directly obtained from the average fleld variations and the continuity of the tangential electric fleld. The average plasma density in the current sheet and lobes, and average lon plasma temperature in the tall, on the other hand, can be derived from the calculated plasma acceleration and MHD momentum equation.

The reconstructed $(*)$ coordinate system developed in this study allows us to represent the steady state, average configuration of the Venus magnetotail. As such, the effects of time-varying upstream conditions have been ramoved, and the resultant variations determined with our analysis can be treated as though they were derived for constant (average) upstream interplanetary conditions. In this coordinate system the consistent steady stite plasma properties of the Venus magnetotall can be calculated, approximately, from our average magnetic field data. The MHD momentum equation in the two-dimensional $X-Y^{*}$ plane of the fleld draping 18 


$$
\begin{aligned}
& \left(\underset{X}{\operatorname{s}} \underset{\mathrm{B}}{)_{X}}=-J_{Z} B_{Y}=\rho\left(v_{X} \frac{\partial}{\partial X} v_{X}+v_{Y} \frac{\partial}{\partial Y} v_{X}\right)\right. \\
& \left(\underset{J}{J} \underset{)_{Y}}{ }=J_{Z} B_{X}=\rho\left(v_{X} \frac{\partial}{\partial X} v_{Y}+v_{Y} \frac{\partial}{\partial Y} v_{Y}\right)+\frac{\partial P}{\partial Y}\right.
\end{aligned}
$$

The pressure gradient term has been dropped in equation 3.1 a becsuse there is no boundary confining the plasma in the $-x-d i r e c t i o n$ as there are in the $\pm Y$-directions, and the plasma is therefore free to flow downtall. For simplicity, we have dropped the $(*)$ from all $Y$ terms in equation 3.1 and throughout this section; however, it should be understood that all equations and discussions in this section refer to the average, steady state configuration described by the statistical $X-Y *$ coordinate system.

If 1) the plasma pressure in the current sheet greatly dominates the magnetic fleld pressure ( $(\gg) 1), 2$ ) the opposite condition holds in the lobes $(\beta \ll 1)$, and 3 ) Y-directed accelerations are not too large, then the $\mathrm{Y}$ - $d$ irected $\mathrm{J} \times \underline{\mathrm{B}}$ force in equation 3.1b will be primarily balanced by che gradient in the plasma pressure alone rather than the inertial forces. In this case virtually all plasma flow will be directed downtall and $v_{Y}(X, Y) \approx 0$. Therefore equation 3.1 a simplifies to Include orily the firse of the two spatial derlvative terms. This sort of tall configuration is expected if ton pick-up $\left(e . g ., O^{+}\right)$is an important physical process In the extended dayside lonosphere, or if solar wind plasma flowing near to the stagnation point on the dayside of Venus flows around 
the obstacle and into the magnetotall. This 1 imit of the equation w111 be explored quantitatively in this section.

Unfortunately, even the large statistical data set used in this study is insufficient to accurately determine the full twodimensional magnetic fleld vartations. Instead, we have derived the average variations of the tall field in directions perpendicular to these averages. For example, we derive the variations of the field with $Y$ in an average sense in $X$. The task at hand now is to use these average variations to derive the average tall plasma propertles. In general, the average value of a product of two functions does not equal the product of the average values of these functions. However, when one or both of the functions is approximately constant over the direction of averaging, these two types of averages are approximately equal.

The $Y$-averaged variation of the downtall velocity, $\left\langle v_{X}(X)\right\rangle_{Y}$, is derived from the continuity of the tangential electric field, or equivalently, the conservation of magnetic flux. The tangential electric field is

$$
E_{Z}=-v_{X} B_{Y}+v_{Y} B_{X}
$$

and is constant and perpendicular to the two-dimensional plane of the magnetic field draping. $E_{Z}$ is roughly equal to the product of the $x$-component of the magnetosheath plasma velocity and the magrietosheath magnetic field Y-component. The average magnetis fleld crossing the flanks of the tall is, from Figure $3.11,3.5 \mathrm{nT}$. 
The average flow speed in the magnetosheath along the flanks of the magnetopause, tallward of $\sim 4 R V, 1 s>90 \%$ of the upstream solar wind speed, and 1 s directed along streamlines which are very nearly pointing in the -X-direction [Sprefter and Stahara, 1980]. For this study we w111 assume the tallward magnetosheath velocity near to the magnetopause from -8 to $-12 R_{V}$ to be $440 \mathrm{~km} / \mathrm{sec}$. $E_{Z}$, therefore, is simply $440 \times 3.5=1.54[\mathrm{mV} / \mathrm{m}]$.

In the limit studied here, $v_{Y} \approx 0$ and equation 3.2 becomes simply $-v_{X} B_{Y}=1.54 \quad[\mathrm{mV} / \mathrm{m}] . \quad B_{Y}$ is approximately constant as a function of the $Y$ location, varying by only $\pm 15 \%$. Therefore, to within the accuracy of this derivation, the $v_{X}$ and $B_{Y}$ terms of this equation can be averaged separately over $Y$. This procedure yields the equation for the average tallward velocity

$$
\left\langle v_{X}(X)\right\rangle_{Y}=\frac{-1540}{\left\langle B_{Y}(X)\right\rangle_{Y}}=\frac{-1540}{11.53+.68 X}
$$

where the velocity is given in $\mathrm{km} / \mathrm{sec}$, and $X$ is in $R_{V}$. This function is plotted in Figure 3.16 from -8 to $-12 R_{V}$ which is the range over which it is reasonably rellably determined.

Extrapolation of equation 3.3 outside the range over which our data set extends is problematic. The qualitative result, however, that tallward plasma motion near Venus is quite slow compared to the solar wind speed, is well determined. The deep tall region from -8 to $-12 \mathrm{R} V$ is shown in this study to be an interesting transition region. Tallward of $-8 R_{V}$ the average plasma velocity increases 


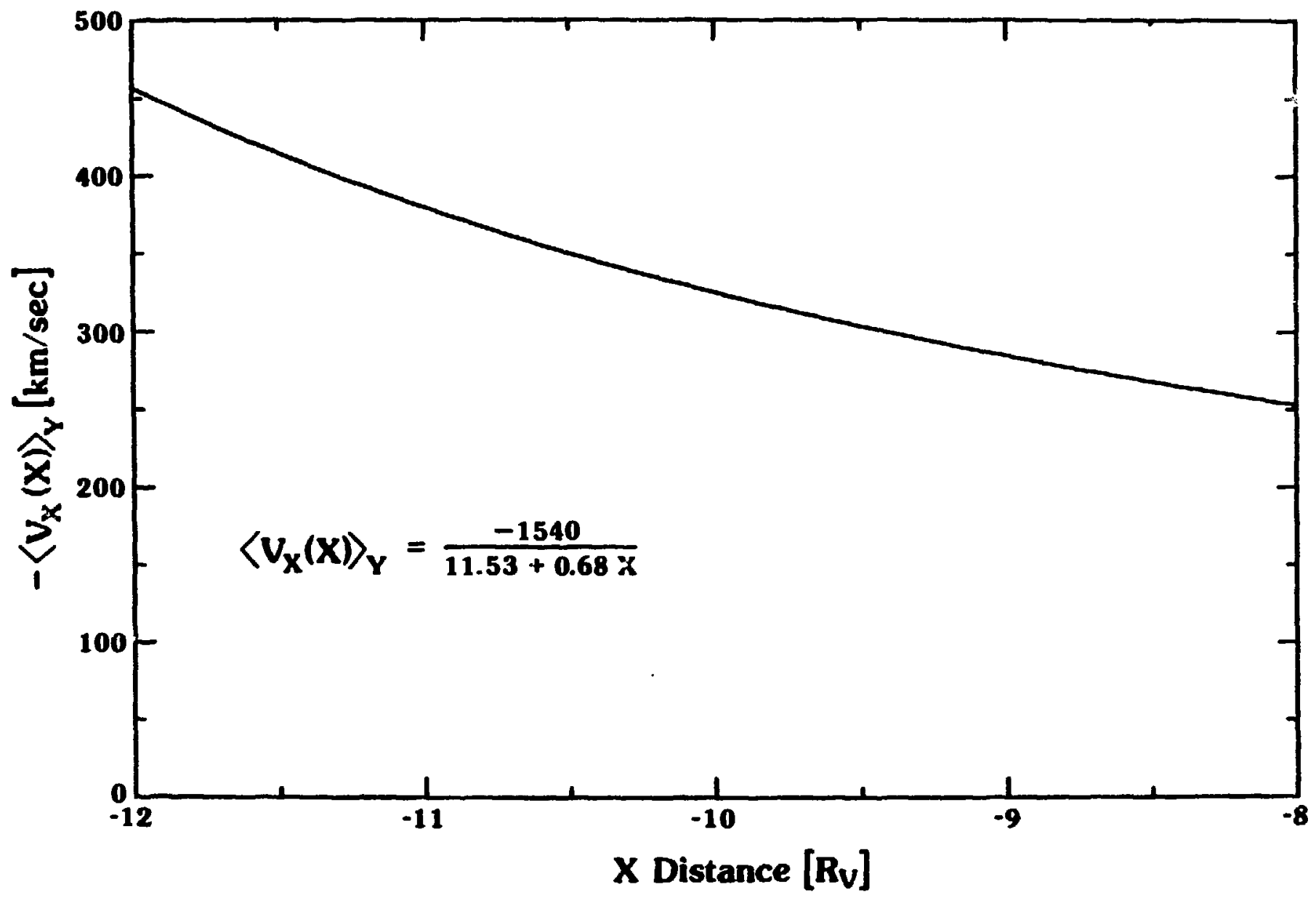

Fig. 3.16. The $Y$-averaged tailward plasma velocity, $\left\langle v_{X}(X)\right\rangle_{Y}$, as a function of downtail distance. Equation 3.3 describes this variation quantitatively. Venusward of $-11.8 \mathrm{R}_{\mathrm{v}}$, the flow is slower than the nominal solar wind speed, while beyond this distance the fleld lines are catching up with the nominally straight IMF configuration. 
from about $-250 \mathrm{~km} / \mathrm{sec}$ to the solar wind speed of $-440 \mathrm{~km} / \mathrm{sec}$ at - -11.8 $R_{V}$, and therefore the draped fleld throughout this portion of the tail continues to fall further behind the IMF. At $-12 R_{V}$ the average plasma velocity is greater than the solar wind speed, and the field in this portion of the tall is in the process of catching up with the comparatively straight IMF configuration.

The steady state plasma acceleration is simply the spatial derivative $v_{X} \partial v_{X} / \partial X$. The Y-averaged spatial acceleration, $\left\langle v_{X} \partial v_{X} / \partial X\right\rangle_{Y}$, cannot be uniquely determined from equation 3.3; however, the product of the average terms in the acceleration gives

$$
\left\langle v_{X}\right\rangle_{Y} \partial\left\langle v_{X}\right\rangle_{Y} / \partial X=\frac{-267}{(11.53+.68 X)^{3}}
$$

in $\mathrm{km} / \mathrm{sec}^{2}$, where $X$ is in $R_{V}$. This spatial acceleration is shown in Figure 3.17 from -8 to $-12 R_{\mathrm{y}}$.

The precise plasma density distribution and temperature cannot be uniquely calculated from the average velocity and acceleration derfved above. The approximate values of these quantities, however, can be calculated from the variations and the momentum equation. In the limit described in this study, equation 3.1 becomes

$$
\begin{aligned}
& -J_{Z} B_{Y}=\rho\left(v_{X} \partial v_{X} / \partial X\right) \\
& J_{Z} B_{X}=\partial P / \partial Y
\end{aligned}
$$




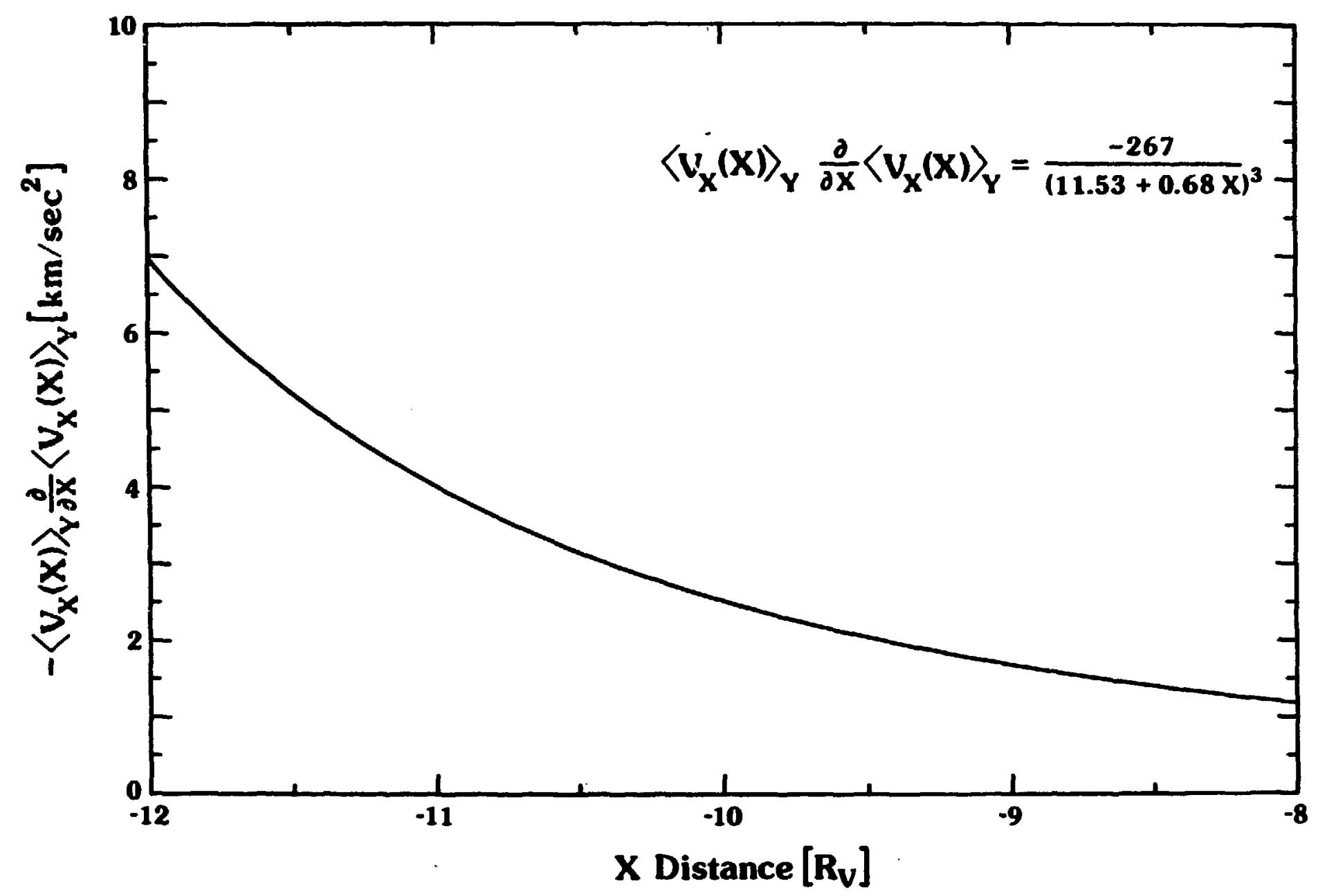

Fig. 3.17. The Y-averaged plasma acceleration $\left\langle v_{X}\right\rangle{ }_{Y} \partial\left\langle v_{X}\right\rangle=\partial X$ as a function of downtail distance. Equation 3.4 describes this variation quantitatively. 
Insertion of the average fleld, currents, and plasma acceleration Into equation 3.5 a ylelds

$$
\rho=\frac{\left\langle-J_{Z}\right\rangle_{X}\left\langle B_{Y}\right\rangle_{X}}{\left\langle v_{X}\right\rangle_{Y} \partial\left\langle v_{X}\right\rangle_{Y} / \partial X}
$$

By averaging the denominator of equation 3.6 over $X$, the remaining $Y$ varlation of the numerator then determines the gross $Y$ variation of the density. of course the plasma acceleration also varies as a function of $Y$; but, since the average plasma velocity is similar to the sheath velocity in this portion of the tall, the acceleration is probably not a strong function of $Y$. In addition, it must be remembered that these are only rough calculations, and that all terms cannot be fully treated with the average varlation information gathered in this study. In the draped lobes the derived mass density is then $\sim 1.2 \times 10^{-22} \mathrm{~kg} / \mathrm{m}^{3}$ while the average derived mass density in the current sheet is $\sim 1.6 \times 10^{-21} \mathrm{~kg} / \mathrm{m}^{3}$. These values are equivalent to densities of approximately .07 and .9 protons $/ \mathrm{cm}^{3}$, respectively. If the plasma in the Venus tall is comprised of $0^{+}$, then the equivalent densities are .005 and $.06 \mathrm{~cm}^{-3}$.

These average values of the plasma density are in good agreement with the lack of observations of plasma in the Venus magnetotall by the PV0 plasma analyzer [Intr1ligator et al., 1980]. This Instrument was principally designed for solar wind observations and does not have the sensitivity to measure plasmas with densities of $\leq 1 / \mathrm{cm}^{3}$ [Saunders et al., 1985]. Since our derived average plasma 
denstites are lower than this cutoff, we would predict that the Venus tall would generally be indlcated by a "plasma dropout" of the plasma analyzer. This is actually observed to be the case [Mihalov and Barnes, 1982]. When present, these observations usually show both $\mathrm{O}^{+}$and proton peaks In the spectra, but the spectra are almost never well enough formed to actually derlve moments from. Even where it is possible to derive moments, these would represent unusual plasma conditions in the tal1 (perhaps high density and low flow speed) while our derived plasma properties are consistent with the average, steady state configuration of the tall.

The typical plasma temperature in the Venus tall can be derived from equation 3.5 by using the variation in the density which was just determined. We derive only the approximate plasma temperature averaged over this entire portion of the tall since the variations derived in this study are insufficlent to determine gradients in the temperature. Averaging all terms in equation $3.5 \mathrm{~b}$ and replacing $\partial \mathrm{P} / \partial \mathrm{Y}$ with $\mathrm{kT} \Delta \mathrm{n} / \Delta \mathrm{Y}$, we solve for the temperature which gives

$$
T=\frac{\left\langle\left\langle J_{Z}\right\rangle_{X}\right\rangle_{Y}\left\langle\left\langle B_{X}\right\rangle_{X}\right\rangle_{Y}}{k} \frac{\Delta Y}{\Delta n}
$$

where $k$ is Boltzmann's constant and $\Delta n$ and $\Delta Y$ are typical variations In density and $Y$ location between the lobes and current sheet. equation 3.7 was numerically evaluated using $\left\langle\left\langle B_{X}\right\rangle_{X}\right\rangle_{Y}=10$ nT and the derived variation in the density between the draped lobes and the current sheet. If the tall is assumed to consist entirely of 
hydrogen, then the proton temperature is of the order of $6 \times 10^{6} \mathrm{~K}$. If, on the other hand, the plasma is principally formed by $0^{*}$, then the Ion temperature is of the order of $9 \times 10^{7} \mathrm{~K}$. These values represent upper bounds only since we have assumed that the electron pressure is negligible in this firal portion of the derivation. If the electron temperature is comparable to or greater than the fon temperature, these values would be much redured. In addition, successive steps in this derivation have required successively greater assumptions, and therefore these derived temperatures are the least well determined of our plasma parameters. We principally intend that they be used only as rough estimates. These values, however, are not so high as to be impossible to achleve in an Induced magnetotall where lon pick-up may be important. $0^{+}$plckedup by the unimpeded solar wind can have temperatures as high as $\sim 2 \times 10^{8} \mathrm{~K}$ when they are picked-up with an initial perpendicular (thermal) velocity of the typical solar wind speed $(440 \mathrm{~km} / \mathrm{sec})$.

Ising the approximate plasma parameters derived above, the lobe and current sheet $\beta$ s can be calculated. The plasma $\beta$ in the lobe is $\sim .08$ which is consistent with the difference in the magnetic flux in the two lobes moving the current sheet from side to side, and with our assumption of low $\beta$ lobes in the above derivation. The $B$ in the current sheet, on the other hand, is $\sim 12$ which is consistent with our assumption of a high $B$ current sheet in the derivation of these plasma parameters. The calculated gyrorad11 of protons and $\mathrm{O}^{+}$1ons, calculated from these plasma moments, are $\sim .1$ and $\sim 1.5 R_{V}$ in the current sheet, respectively, and only $\sim 30 \%$ of that in the lobes. 
Since these size scales are appreclably smaller than the width of the tall, our use of the fluid momentum equation in this derivation is vindicated.

The average plasma propertles derived in this study can be used to determine the approximate average mass flux down the Venus tall which also represents an upper bound on the mass loss rate of Venus. For this rough calculation we assume that the current sheet and lobes each take up $1 / 3$ of the cross-sectional area of the tall, which we assume to be circular and $5 \mathrm{R}_{\mathrm{V}}$ in diameter. At the average downtall distance of data in our data set $\left(-10.8 \mathrm{R}_{\mathrm{V}}\right)$, equation 3.3 gives $\left\langle v_{X}\right\rangle_{Y}=-368 \mathrm{~km} / \mathrm{s}$. Multiplying this velocity by the sum of the current sheet and lobe densities times their respective crosssectional areas, we calculate a downtall mass flux of $\sim 1 \times 10^{26} \mathrm{amu} / \mathrm{s}$ or $\sim 6 \times 10^{24} \mathrm{o}^{+} / \mathrm{s}$. If all of the material in the Venus magnetotall is of planetary origin, then this value represents the approximate Venus mass loss rate. In any case, $\sim 1 \times 10^{26} \mathrm{amu} / \mathrm{s}$ represents an upper bound for the mass loss rate of the Venus atmosphere through tall formation.

\subsection{Summary}

Th1s study began with the schematic diagram of the solar wind/IMF interaction with Venus displayed in Figure 3.1. We postulated that the IMF $X$-component could have an Important effect on the Internal structure of the draped magnetotall. In particular, varlations of the $x$-component could cause the flapping of the current sheet from side to side within the tall consistent with the 
magnetic pressure of the varylng quantities of magnetic flux in the two lobes. We developed this chapter in three sectlons.

In Section 3.2 we demonstrated that magnetometer data ordered by spatial location is extremely variable. Both lobes and the current sheet can be found at all spatial locations within the tall, although there is a preference for the tallward pointing lobe on the $+Y$ side and the Venusward pointing lobe on the $-Y$ side. The average separation between the two lobes was shown to be offset toward the lobe which contalns less magnetic flux. This observation, along with the high varlability observed, confirmed our postulated Importance of the IMF $X$-component. Clearly, what was needed to further advance the quantitative study of the Venus tall was to find a coordinate system which measures locations with respect to the Internal tafl structures themselves.

In Section 3.2 we also showed that magnetic reconnection is a small effect in the central venus tall, and that the magnetic fleld strength in the tall, referenced to the average magnetosheath value Immediately adjacent to the tail, at the points in each orbit where the PVO pierces the megnetopause, is strongly correlated with the magnetic fleld draping angle. In the lobes the field points roughly tallward and Venusward, and the relative fleld strength is large. In the lobe separating current sheet, on the other hand, the fleld points roughly across the tall and the relative fleld strength is comparatively small. We used chis correlation to construct a coordinate system which statistically locates data with respect to the tall current sheet itself。 
In Section 3.3 we actually constructed this coordinate system and measured the average varlations of the fleld components for the first time. The $\mathrm{B}_{X}$-component was demonstrated to smoothly vary from strongly Venusward on the far $-Y$ side of the tall, through zero in the center of the current sheet, to strongly tallward on the far $+Y$ side of the tall. The $\mathrm{B}_{\mathrm{Y}^{*}}$-component, on the other hand, showed a two-humped distribution across the tall and decreased falrly linearly with distance from the planet. From these variations we drew the average draping pattern in the deep Venus tall in Figure 3.12 and derived the average cross tall current density. distribution. Knowledge of the average magnetic fields and selfconsistent currents made it possible to calculate the electromagnet1c $J_{\times} \mathrm{B}$ forces and examine the average consistent plasma properties of the tall.

In Section 3.4 we used the continulty of the tangential electric field and found field variations to derive the average tallward velocity and acceleration as functions of downtail distance. The tallward veloclty was shown to vary from $\sim-250 \mathrm{~km} / \mathrm{sec}$ at $-8 \mathrm{R}_{\mathrm{V}}$ to $\sim-470 \mathrm{~km} / \mathrm{sec}$ at $-12 \mathrm{R}_{\mathrm{V}}$. From the derived accelerations, the calculated $\mathrm{J} \times \mathrm{B}$ forces, and the MHD momentum equation, we calculated the approximate plasma densities in the current sheet and lobes, and very approximate average plasma temperature. The current sheet density was shown to be $\sim .9 \mathrm{p}^{+} / \mathrm{cm}^{3}\left(.060^{+} / \mathrm{cm}^{3}\right)$ while the lobe density was approximately $15 \%$ of that. These densities were shown to be consistent with the general lack of PVo plasma observations in the tall. If the tall plasma is composed purely of hydrogen, then 
we calculate an average, approximate, maximun ion temperature is $\sim 6 \times 10^{6} \mathrm{~K}$; while if it is composed essentially of oxygen, this temperature is $\sim 9 \times 10^{7} \mathrm{~K}$. Finally, we calculated the mass flux in the tail which is $\sim 1 \times 10^{26} \mathrm{amu} / \mathrm{s}$. This value represents an upper bound for the mass loss rate of the Venus atmosphere through tall formation.

While this study derives many previously unknown average quantitles of the deep Venus magnetotall, and sets, for the first time, the average magnetic fleld draping pattern, self-consistent currents and forces, and approximate Inferred plasma properties of this region, it should not be thought of as an end to the study of the deep Venus magnetotall. Rather, a powerful analysis tool has been developed for examining the detalled physics of this region by referencing the location of not just magnetic field data, but all PVo data, with respect to the internal tall structures themselves. Finally, our method may be very valuable in examining the magnetotalls of other mass loading obstacles in the solar wind, such as comets, and the deep (reconnected) terrestrial magnetotall, since the orfentation of the upstream IMF should also have a strong Influences on these reglons.

\subsection{Chapter Acknowledgments}

We gratefully acknowledge valuable discussions with D. N. Baker, R. C. Elphic, J. G. Luhmann, W. I. Newman, J. L. PhIllips, G. L. S1scoe, and D. T. Young. We also gratefully acknowledge valuable constructive critiques from J. R. Spreiter and 
another (anonymous) referee. The magnetlc fleld data used in this study and work done at the IGPP, UCLA were supported under NASA contract NAS2-9491. One of the authors (H.E.S.) was supported by the Office of Naval Research, while another (M.A.S.) was supported by an ESA Research Fellowship. The princlpal author and work done at Los Alamos were supported by Los Alamos National Laboratory under the auspices of the Un1ted States Department of Energy. 
The Glacob1n1-21nner Magnetota11

Ta11 Configuration and Current Sheet

\subsection{Chapter Overview}

The high resolution plasma electron and magnetic ileld data sets from the ICE tal: traversal of Comet Glacobinf-ZInner have been combined to make a detalled study of the draped G-Z magnetotail in general, and its field reversing current sheet in particular. The geometry of the magnetotall at the time of the ICE crossing is determined, and is shown to be consistent with a clrcular tall cross-section rotated $10.5^{\circ}$ in the normal sense of aberration and 9.9 $9^{\circ}$ above the ecliptic plane, blsected by a cross tall current sheet which is rotated $43^{\circ}$ out of the ecllptic about the solar wind velocity vector. MHD continuty, momentum, and energy equations are combined with the plasma and fleld observations to determine unmeasured plasma properties at ICE and upstream at the average point along each streamiline where the cometary lons are picked-up. The fon temperature, beta, and flow speed at ICE range from $1-1.5 \times 10^{6} \mathrm{~K}, 1-4$, and -20 to $-30 \mathrm{~km} / \mathrm{sec}$, respectively, in the draped lobes to $\sim 1.2 \times 10^{5} \mathrm{~K}$, up to $\sim 40$, and $\sim-20 \mathrm{~km} / \mathrm{sec} 1 \mathrm{n}$ the current sheet. Upstream at the average pick-up locations, the f?.ow velocity, ion temperature, density, and lon source rates range from $\sim-75 \mathrm{~km} / \mathrm{sec}, \sim 4 \times 10^{6} \mathrm{~K}, \sim 20 / \mathrm{cm}^{3}$, and $\sim 1.5 / \mathrm{cm}^{3} / \mathrm{sec}$ in the regions 
upstream from the lobes to $\sim-12 \mathrm{~km} / \mathrm{sec}, \sim 1 \times 10^{5} \mathrm{~K}, 200-600 / \mathrm{cm}^{3}$, and $\sim 3.6 / \mathrm{cm}^{3} / \mathrm{sec}$ in the prime mass loading region upstream from the current sheet. Gradients in the plasma properties at the edges of this region are quite strong, and the diameter of the region is $\sim 1500 \mathrm{~km}$, in good agreement with the expected size scale of the cometary Ionopause; Implications of our Inferred plasma properties In this region are examined. The derived and measured flow velocity and measured plasma density at ICE are combined to calculate the transport rate of lons past the plane of the ICE encounter, and we calculate that $\sim 2.5-5 \times 10^{28}$ ions/sec (neutrals/sec in the steady state) are produced by the comet with only $\sim 1 \%$ of these traveling down the magnetotail.

\subsection{Introduction}

Man has observed apparitions of comets and visible comet tails over many millennia and wondered about their origin. Three and one half decades ago Blermann [1951] postulated the first modern scientific explanation of visible cometary tails, namely that they are caused by the Interaction of an ionized gas of solar origin (the then unknown solar wind) with the comet. Alfven [1957] then Introduced the concept of magnetic field draping as an important aspect of the physical process for comet magnetotall formation. Since that time much work has been done on refining the understanding of the solar wind interaction with comets in general, and the formation of cometary talls in particular, and insights into the nature of the solar wind interaction with comets have become 
highly refined (see reviews by Brandt [1982], Ip and Axford [1982], and Mend1s and Houp1s [1982], and references there1n).

During the past year the first in situ observations of comets have been made so that actual measurements are finally avallable for detailed analysis. On September 11, 1985 the International Cometary Explorer (ICE) spacecraft traversed Comet Glacobini-Z1nner (G-Z) $7800 \mathrm{~km}$ downstream from the nucleus. More recently, observations have been made at Comet Halley by the Glotto, Sakigake, Sulsel, and two Vega spacecraft. However, all of the Halley encounters occurred along trajectorles upstream from the cometary nucleus. While these various Halley encounter data sets are already proving to be Invaluable in further unravelling many of the mysterfes of the detailed interaction of the solar wind with cometary bodies, none can directly address the most noticeable element of cometary apparitions: the visible lon tail. The ICE data, therefore, provides a unique opportunity for the detailed in situ examination of a cometary magnetotall.

A number of recent studies have discussed various aspects of the ICE magnetotail data. Papers in the spectal issue of Sclence by Bame et a1. [1986], Smith et a1. [1986], and Meyer-Vernet [1986a] describe the global variations observed throughout the ICE encounter Interval by the plasma electron, magnetometer, and radio wave experiments, respectively. More detalled analyses of these three data sets have been carried out by Zwickl et a1. [1986], Slavin et a1. [1986a], and Meyer-Vernet [1986b], respectively. Flnally, Siscoe et al. [1986) combine approximate field and plasma values for 
four locations across the tall in order to derive approximate values for certain unmeasured plasma properties such as the lon pick-up speed. In the present paper we combine all three data sets at their highest time resolutions in order to make detalled analyses of the Glacobini-zinner magnetotall.

Magnetic field data on ICE were obtained by the Jet Propulsion Laboratory magnetometer [Frandsen et al. 1978], which provided three orthogonal vector measurements every $1 / 3$ second during the ICE encounter. Plasma electron measurements on ICE are supplied by the Los Alamos plasma analyzer [Bame et al. 1978] and certain electron moments can be derived from the radio noise spectrum measurements of the Observatoire de Paris/GSFC radio wave experiment [Knoll et al. 19781. At the ICE encounter telemetry rate, the Los Alamos electron spectrometer measures the full two-dimensional electron distribution function (integrated over $\pm 65^{\circ}$ about the equatorial plane) in one spacecraft rotation ( 3 seconds) once every 24 seconds. The derivation of electron density and temperature from the radio wave experiment data stems from plasma noise spectroscopy, and has been described in detail by Meyer-Vernet [1986a]. This experiment provides measurements at Intervals which vary from 3 to 18 seconds and such measurements are allased in time over variable intervals, ranging from 1.5 to 18 seconds. In addition, the electron spectromecer, which was designed to measure the solar wind from a halo orbit at the earth's Ll point, has a minimum energy level of $\sim 10 \mathrm{eV}$ except when taking data in a special photoelectron mode. This means that the distribution functions of the highest density 
and lowest temperature plasma in the center of the magnetotall current sheet cannot be fully determined. The radio experiment, on the other hand, is most accurate for these colder, more dense plasmas. Since 1) the denstty ranges from about $50 / \mathrm{cm}^{3}$ to over $650 / \mathrm{cm}^{3}$ and electron energles range from $\sim 1 \mathrm{eV}$ to $\sim 10 \mathrm{eV}$, 2) variations in the fleld and plasma properties occur on time scales which range from a fraction of a second to a few minutes, and 3) the electron and field properties are Inexorably linked, the three data sets combined in this study are highly complementary.

In this study these complementary data sets are combined to make detailed analyses of the configuration and properties of the Glacobini-Zinner magnetotall. We develop this study in sections. In the next section (4.2) the global interaction of the solar wind and embedded interplanetary magnetic field (IMF) with the comet is described as it pertains to magnetota1l formation. The extrapolated solar wind flow direction and magnetotall entry, exit, and current sheet crossing times are used to determine the location and dimensions of the tall, and these results are combined with the average magnetic field drapting direction to infer the internal tall structure. In Section 4.3 the pressure balance and plasma acceleration conditions are dertved from the MHD equations, and their implications for the plasma properties further upstream in the region near to the comet nucleus are discussed. In Section 4.4 the particle flux is integrated over the magnetotall for the entire cometary encounter, and these results are used to determine the Importance of comet tall formation for the removal of the lonized 
cometary efflux. Finally, In Section 4.5, the 1mplications of the varlous properties derived in this study are examined, particularly with regards to the upstream near- nucleus region where the tail formation process occurs.

\subsection{Ta11 Formation and Topology}

The basic physical processes responsible for cometary magnetotall formation are slowing of the solar wind flow by mass addition from the extended cometary atmosphere and the consequent draping of the interplanetary magnetic field about the cometary obstacle. The solar wind essentlally flows radially outward from the sun carrying with it an embedded interplanetary magnetic fleld (IMF). As the flow nears the comet nucleus it becomes slowed and significantly mass loaded. This mass loading process is an Integrated effect in that the density of cometary fons at any location is a function of the total mass addition along the streamline sunward from the observation location. Conductivity of the Ionopause boundary itself at least partially excludes the magnetic fleld from the region immedtately surrounding the nucleus, and thereby serves to divert the flow around the obstacle. Less mass is added to the flow along streamlines which pass progressively farther from the comet nucleus since the neutral cometary material avallable for lonization drops off as a function of radial distance.

Since the magnetic field 1inks the reglons of greater and lesser slowing, the field becomes draped about the cometary obstacle. The magnetic fleld in the region of greatest integrated 
mass addition, and therefore slowest flow, becomes highly bent and forms the field reversing, cross tail current sheet in the cometary magnetotail. The fleld in regions of progressively less mass addition, and therefore greater flow speed, immediately adjacent to the current sheet becomes highly draped into the sunward and antisunward directions, forming the tail lobes on elther side of the current sheet. The narrow, high density current sheet within the cometary magnetotail is also probably the reglon observed from earth as the visible fon tall. Slavin et ai. [1986b] have compared the ICE data and ground based observations and show that there 1 s good agreement between the highest density region measured at ICE and the brightest region observed from the earth.

The top panel of Figure 4.1 shows a schemat1c representation of the draped magnetic field configuration about G-Z profected into the plane defined by the average upstream magnetic field and solar wind flow vectors. For simplicity this figure is drawn for an IMF which has no $X$-component. While the $X$-component modifies the relative sizes of the two draped tall lobes in the deep Venus magnetotall [McComas et a1. 1986a], and also probably at comets, the fundamental draping process only involves the component of the field perpendicular to the plasma flow direction.

In the magnetotail formation process just described, the orlentations of the solar wind velocity vector and upstream magnetic fleld clearly play important roles in ordering the magnetota11 location and internal configuration. The most reasonable coordinate system for studying the G-Z magnetotall 1s, therefore, based on 


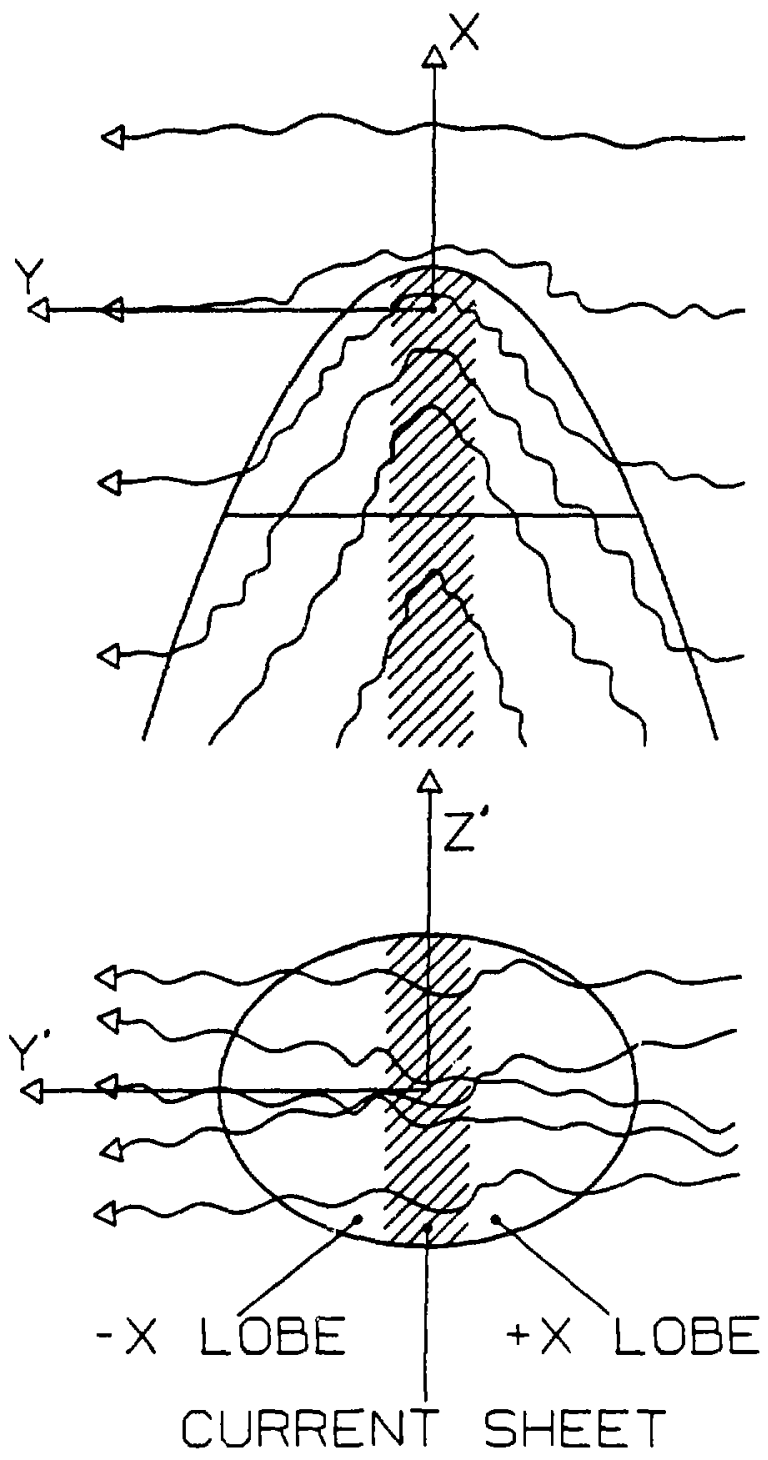

Fig. 4.1. Schematic diagram of the draped magnetic field configuration in the Giacobini-Zinner magnetotall projected into the $Y^{\prime}-X$ and $Y^{\prime}-Z^{\prime}$ planes. The $X$ axis is defined to be antiparallel to the average solar wind flow direction for the crossing while the $Y^{\prime}$ axis is defined to be parallel to the average magnetic field direction in the plane perpendicular to the flow. Waves associated with pick-up instabilities cause the accreted field in the tail to be highly kinked and the fleld orientation in the tail to be quite variable as a function of location and time. Nonetheless, these variations do not stop the formation of the global tail structures such as the current sheet (cross hatched), two tail lobes, and magnetotail boundary (solid smooth curves). The horizontal line in the top panel indicates the location of the cut through the tail represented by the elliptical tail cross section (see text) drawn in the bottom panel. 
these directions. In the coordinate system used in this study (denoted the $X-Y^{\circ}-Z^{-}$system) the solar wind veloctty vector is aligned antiparalleI to the X-axis. The $Y^{\circ}$-axis is allgned with the average magnetic fleld vector crossing the tall in the plane perpendicular to the $\mathrm{X}$-axis. Finally, the $\mathrm{Z}^{-}$-axis completes the right-handed coordinate system, and lies in the plane of the current sheet since this plane is perpendicular to the primary curl of the field lines (In the $X-Y^{\circ}$ plane). The lower panel of Figure 4.1 is a schematic representation of the magnetic fleld projected into the $Y^{\circ}-Z^{\circ}$ plane. Portions of the Instantaneous fleld in this plane may point In directions very different from the average, $\mathrm{Y}^{-}$, direction. These variations are caused both by fluctuations in the fleld in space and time due to upstream waves and instabilities assoclated with the cometary ion pick-up process, and actual rotations of the upstream IMF. It is still, however, the global, average magnetic orlentation which dominates the essential tall structure.

One of the important new results from the $G-Z$ encounter is the large amount of plasma [Bame et a1., 1986; Gosling et a1., 1986] and fleld [Smith et al., 1986, Tsurutani and Smith, 1986a and b] turbulence assoclated with the extended cometary ion pick-up region. These fluctuations cause intense kinking of the fleld and large spatial variations of the plasma properties. In Figure 4.1 we attempt to show the effect of these varlations on the magnetotall. Since the flow is substantially slowed and compressed within the magnetota11, upstream varlations along streamlines passing near the stagnation region also become compressed and incorporated into the 
magnetotall fine structure. ThIs process probably accounts for much of the varlability observed within the ta11, particularly in the high time resolution measurements.

There was no spacecraft upstream from $\mathrm{G}-\mathrm{Z}$ to monftor the solar wind and IMF conditions during the ICE encounter. In this study, therefore, we use extrapolated solar wind observations from the Inbound and outbound portions of the ICE encounter to infer the solar wind conditions at the time of the ICE crossing. Nine point running averages of the plasma flow speed and flow angle (as measured from the GSE -X-axis toward the GSE -Y-axis) are plotted in F1gure 4.2 for the ent1re ICE encounter 1nterval. No data are shown In the central portion of the crossing were the flow speed dropped below $30 \mathrm{~km} / \mathrm{s}$, the minlmum bulk flow speed which can be rellably determined by the Los Alamos plasma electron spectrometer. The dashed IInes indicate the Inferred varlations of the two solar wind parameters in the absence of the cometary interaction. The best extrapolated values for the solar wind speed, ${ }^{v}$, and flow angle, $\phi$, during the tall traversal, at approximately $1100 \mathrm{UT}$, are $v_{S W}=460 \mathrm{~km} / \mathrm{s}$ and $\phi=-10.5^{\circ}$. The X-axis used In this study 1s, therefore, first rotated through an angle of $10.5^{\circ}$ from the GSE Xaxis so as to be antiparallel to the projection of the solar wind velocity vector in this plane.

If the tail cross-section is circular at the downstream distance of the ICE traversal, then the average orientation of the tall at the time of the ICE crossing can be determined. Figure 4.3 shows the geometry under the simplifylng assumption of a circular 


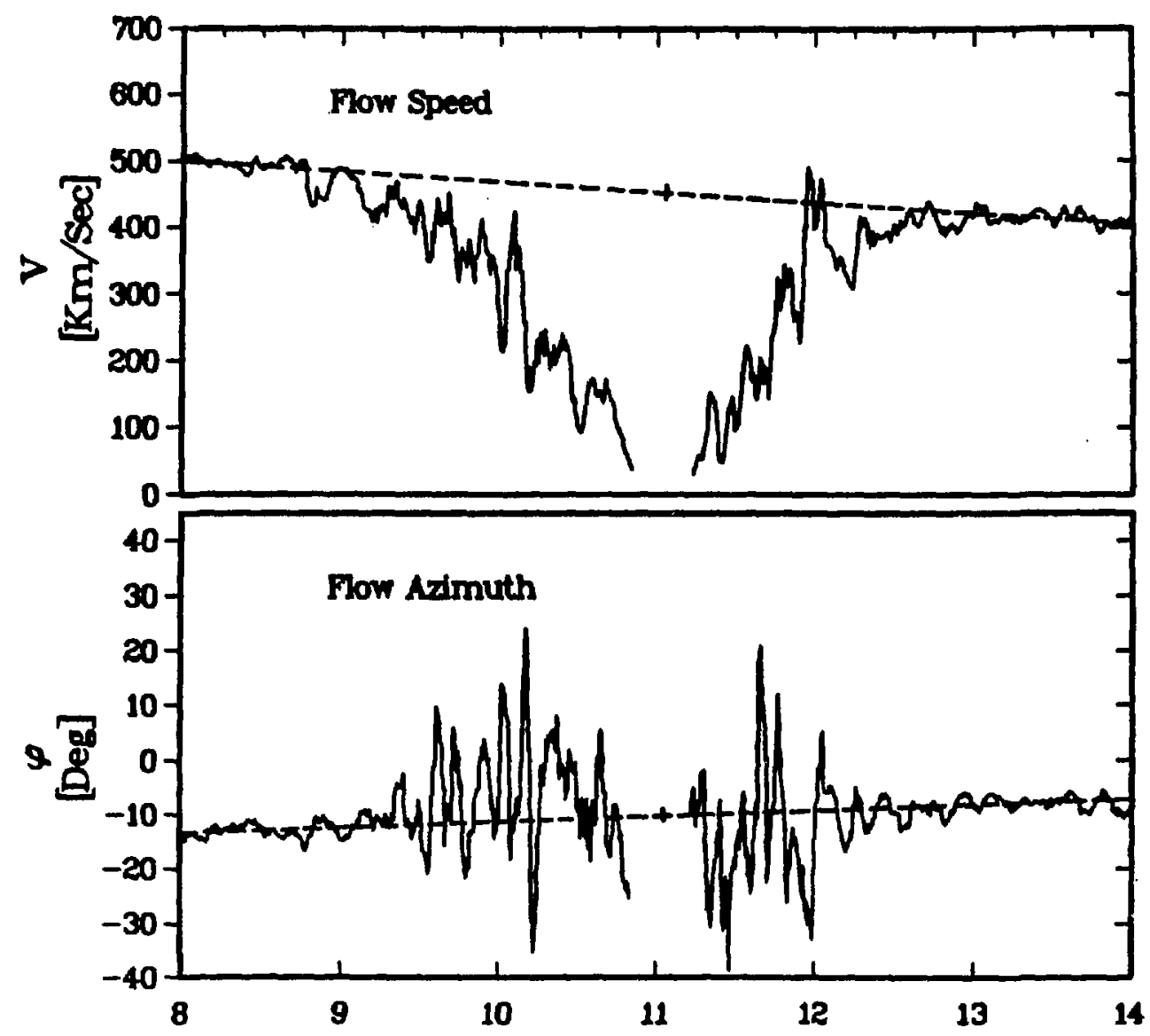

Fig. 4.2. Nine point running averaged flow speed and flow angle for the entire ICE traversal of Comet Giacobini-Zinner. No data are shown in the central portion of the tall where the determination of these parameters from the electron distribution functions is unreliable (flow speeds $<30 \mathrm{~km} / \mathrm{s}$ ). The flow azimuth is measured positive from the solar ecliptic (SE) -X direction toward the SE - Y direction, and therefore a value of $-4^{\circ}$ is consistent with the nominal aberration angle. The dashed lines show interpolations of the upstream solar wind flow values across the tall. Since there was no upstream monitor for the ICE traversal, these extrapolations are used to provide approximate upstream conditions. Vertical tics at $\sim 11: 03$ indicate the center of the tail crossing. 


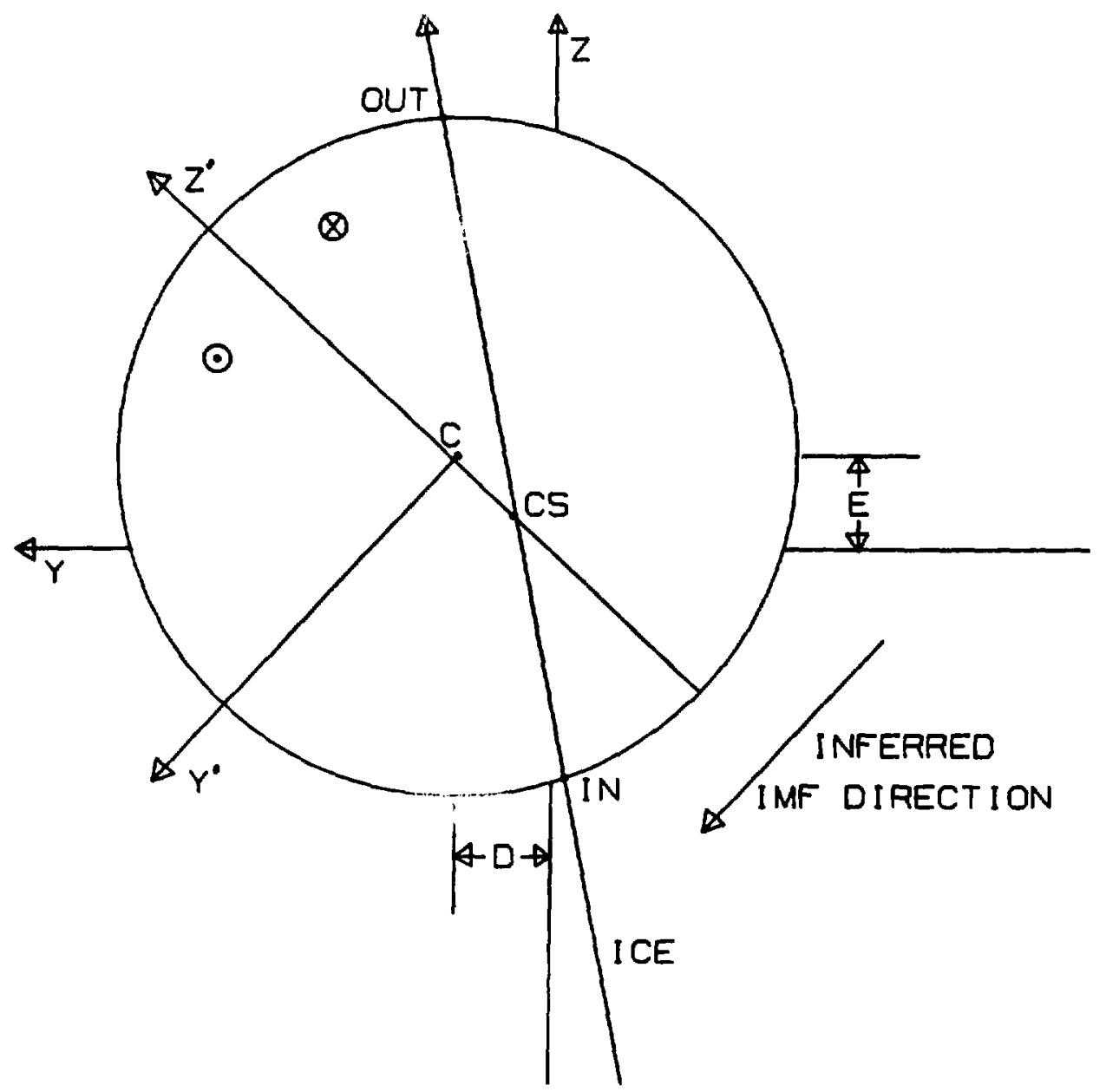

F1g. 4.3. Schematic representation of the ICE tail crossing geometry. The points labeled IN, CS, and OUT are the inbound lobe, current sheet center, and outbound lobe crossings along the ICE trajectory. Point $C$ indicates the inferred center of the tail assuming that the tai: is aberrated a distance $D$ which corresponds to an angle of $10.5^{\circ}$ at $7800 \mathrm{~km}$, and that the tall cross section is circular. The orientation of the $Z^{\prime}$ axis (the current sheet) is independently inferred from the average field orientation ihrough the tail (see text); interestingly, the inferred tall center, $C$, lies very close to the $\mathrm{Z}^{\prime}$ axis. 
cross-section. Polnts "IN" and "OUT" are the tall entry (10:59:40 UT) and tall exit $(11: 07: 40)$ as given by Slavin et a1. [1986a] wh1le "CS" 1 s the current sheet center (11:02:50 UT) as determined in this study. The GSE Y offset distance of the center of the tall, D, is simply the downtall distance of the crossing, $7800 \mathrm{~km}$, mult1plied by the tangent of $10.5^{\circ}$, or $1450 \mathrm{~km}$. Given points IN and OUT on the circle, and that the center must lie at a GSE Y value of $1450 \mathrm{~km}$, the GSE $Z$ offset of the tail, E, can be found. This offset is $1360 \mathrm{~km}$, which corresponds to a solar wind flow directed $9.9^{\circ}$ above the ecliptic. Flow angles of $\sim 10^{\circ}$ are unusual for the solar wind both within and out of the ecliptic plane. For this crossing, however, they may be attributable to the stream/stream Interaction observed by ICE between 04:00 and 06:00, just prior to the comet encounter. The ICE spacecraft traverses the tall slightly off-center in this geometry, and the tail diameter is, therefore, slightly larger than the crossing interval would Indicate. The tall diameter derived by this technique is $10,100 \mathrm{~km}$. If the cross tall current sheet bisects the tall, then it must pass not only through point CS, but also through the tall center. This gives a current sheet orlentation which is rotated $47^{\circ}$ out of the ecliptic plane.

This current sheet orlentation compares quite favorably with the results derived from other more direct methods. Minimum variance analysis of the field rotation through the current sheet ylelds an angle between the ecliptic and the current sheet of $43^{\circ}$ [Slavin et al., 1986a], which is in good agreement with the 
direction expected from the average upstream ortentation extrapolated from the ICE observations. Remote sensing of energetic Ion gyromotions by Daly et al. [1986] 1ndependently determines the average field orlentation, and glves a current sheet Inclination of 46 . All of these average results are in excellent agreement given the large amount of variability of the local field measurements, as Indicated schematically in Figure 4.1 . For the remainder of this study we assume that the angle between the current sheet and the ecliptic plane is $43^{\circ}$. The angle between the ICE trajectory and the current sheet, In the $Y-Z$ plane, is then $35^{\circ}$.

It is interesting that the geometry derived under the circular cross-section assumption Independently yields a current sheet Inclination within only $4^{\circ}$ of that derlved disactly from the fleld observations. The assumption of a clrcular tall cross-section, however, is rather ad hoc since the physical process responsible for determining the tall width is different for directions parallel and perpendicular to the current sheet. In the parallel ( $\left.\mathrm{Z}^{-}\right)$direction, the width should be a direct measure of the effective size of the prime mass loading region. The width in the perpendicular ( $\mathrm{Y}^{-}$) direction, on the other nand, is governed by the draping configuration and flaring angle of the ta.11 lobes as well as the downtall distance of the observations. These considerations assume that the external pressure surrounding the tall is 1sotropic, as suggested by Siscoe et al. [1986]. If there is sufficient flow so that the ram pressure component is still Important along the tall boundary, then this force acts to clrcularize the tall since it is a 
strong function of the flaring angle. For this study we w11: aggume that the crossing geometry is correctly described by Figure 4.3. We have also examined the data under other reasonable geometry assumptions, and find that the resu+ts described here are not particularly sensitive to the exact configuration.

The basic combined data set used in this study is shown in Figure 4.4, and contains data from 10:59 to 11:09 UT. The high resolution magnetic field data (11ght lines) have been averaged (heavy lines) over 3 and 18 seconds centered on the Los Alamos and Observatolre de Paris plasma observations, respectively. In this manner the fleld and plasma measurements correspond to the same averaging Intervals and can be directly compared. Los Alamos plasma data have not been used for the seven points near the center of the tall where the plasma moments are unrellably determined due to observational IImitations. Obvious fluctuations between plasma values derived with the two techniques have been intentionally Included elsewhere since both are presumed tú be accurate in those regions, and the variations therefore give some Indication of the rellability of the values.

\subsection{MHD Equilibrlum}

In this section we combine the ICE plasma and magnetic field observations with the MHD continuity, momentum, and energy equations to calculate certain unmeasured plasma ion properties at the ICE location and Infer some of the conditions upstream along streamlines where substantial mass is being added to the flow. Neglecting terms 

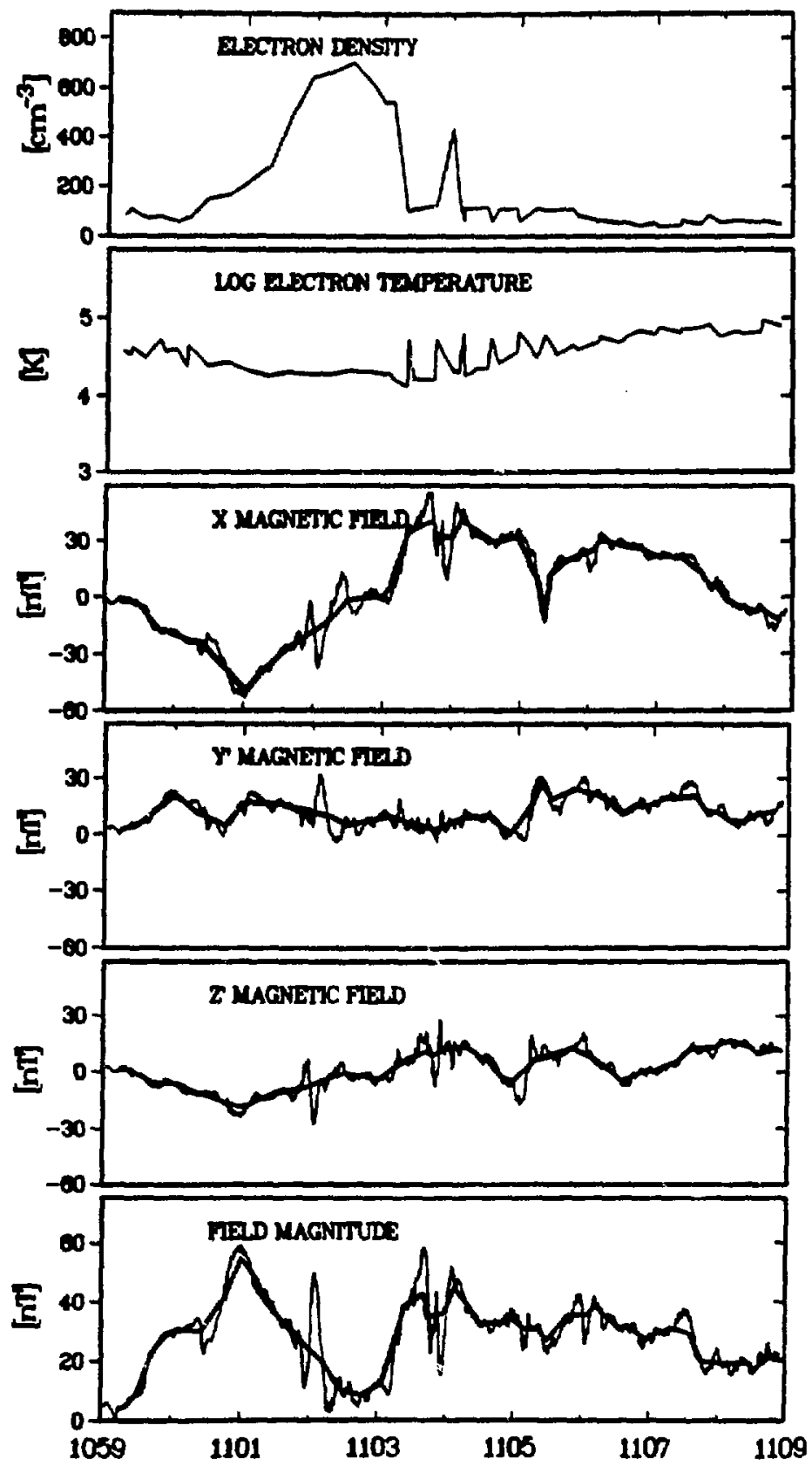

Fig. 4.4. Combined data set used in this study. Electron density and temperature are independently derived from the Los Alamos plasma electron and Observatoire de Parts radio wave data sets, Jet Propulsion Laboratory magnetometer data are shown in the bottom 4 panels in high time resolution (light lines) and averaged over the corresponding plasma measurement intervals (heavy lines). 
due to gravity and following the development of Siscoe [1982], the MHD continuity, momentum, and energy equations are written in conservation form

$$
\begin{aligned}
& \frac{\partial \rho}{\partial t}+\nabla \cdot(\rho \nabla)=s^{*} \\
& \frac{\partial}{\partial t}\left(\rho v+\varepsilon_{o^{\mu}} \tilde{I} \tilde{I}\right)+\nabla \cdot(\tilde{\tilde{P}}+\rho \nu v-\tilde{\tilde{T}})=0
\end{aligned}
$$

$$
\begin{aligned}
& \frac{\partial}{\partial t}\left(\frac{1}{2} \rho v^{2}+U+\frac{\varepsilon}{2} E^{2}+\frac{1}{2 \mu_{0}} B^{2}\right)+ \\
& \nabla \cdot\left[\left(\frac{1}{2} \rho v^{2}+U+\tilde{\tilde{P}}\right) \cdot v+q+\tilde{I}\right]=0
\end{aligned}
$$

where $p$ is the plasma mass density, 18 the plasma velocity, $U$ is the Internal energy, $\dot{\tilde{p}}$ is the plasma pressure tensor, $q$ is the heat flux, $\tilde{I}$ is the Poynting vector, $\tilde{\bar{T}}$ is the Maxwell stress tensor, $\tilde{E}$ and $\tilde{B}$ are the electric and magnetic fields, and $\varepsilon_{0}$ and $\mu_{0}$ are the permitivity and permeability of free space. The mass density source term, $s^{*}$, in the continuity equation (4.1) represents the addition of plck-up lons to the plasma by processes such as photoionization 
and charge exchange, and may be a very complicated and largely unknown function of both location and time.

The right-hand sides of equations 4.2 and 4.3 are Identically zero only if particles are plcked-up from being initially at rest. otherwise, the momentum and energy source terms (right-hand sides of equations 4.2 and 4.3 ) are $S^{\star}$ and $\frac{1}{2} s^{\star} w^{2}$, where is velocity of the neutral flow which is being lonized or charge exchanged, and pickedup by the flowing plasma. Typically, $|\lesssim| \lesssim 1 \mathrm{~km} / \mathrm{sec}$; therefore, ignoring the neutral flow's contributions to the momentum and energy densities is justiflable if pick-up velocities are appreciably greater than $1 \mathrm{~km} / \mathrm{sec}$ and charge exchange is ignored. The flow velocities at the time of pick-up calculated later in this section will be shown to be consistent with this condition across the tail. The divergence of the Maxwel1 stress tensor and Poynting vector terms from equations 4.2 and 4.3 can be rewritten

$$
\nabla \cdot \tilde{\tilde{T}}=\varepsilon_{\rho^{\mu}} \circ \frac{\partial \tilde{I}}{\partial t}+\rho_{c} \tilde{E}+\tilde{J} \times \tilde{B}
$$

and

$$
\nabla \cdot \bar{I}=-\tilde{E} \cdot \bar{J}-\frac{\partial}{\partial t}\left(\frac{\varepsilon_{o}}{2} E^{2}+\frac{1}{2 \mu_{o}} B^{2}\right)
$$

where $\rho_{c}$ is the charge denstty. For the draped magnetotail field geometry described in the previous section, the $\mathrm{Y}^{-}$- and $\mathrm{Z}^{-}$-axes were 
defined such that the average magnetic fleld vector lies in the $X-Y^{\prime}$ plane. In such a coordinate system the average $z^{-}$-directed magnetic field is zero, and varlations of the fleld and plasma properties should be princlpally functions of $X$ and $Y^{\prime}$. Consequently it is justifiable to make the approximations: $\partial / \partial Z^{-}=0$ and $B_{Z}=0$. Another characteristic of an $\mathrm{X}$-aligned magnetotall configuration is that $\partial B_{X} / \partial Y \gg \partial B_{Y} / \partial X$ throughout most of the tall. McComas et a1. [1986a] demonstrate this point, for example, for the Venus magnetota1l, which is also formed by mass loading and magnetic fleld draping of the solar wind and IMF. Including these simplifications, the cross tall current density, $\tilde{j}$, can be rewritten

$$
\tilde{\mathrm{J}} \approx \mathrm{J}_{\mathrm{Z}^{-}} \hat{\mathrm{Z}}^{-} \approx \frac{-1}{\mu_{0}} \frac{\partial \mathrm{B}_{\mathbf{x}}}{\partial \mathrm{Y}^{-}} \hat{\mathrm{z}}^{-}
$$

-hich is expressed purely in terms of measured quantities.

The plasma electron and fleld data avallable from the single ICE tall traversal are clearly insufficient to determine fully all terms in equations 4.1-4.3. Fortunately, these equations can be simplified substantially by making suitable approximations, and the approximate force balances within the tall can be determined. In the pressure term, we assume that the picked-up electrons and lons have had sufficient time to 1sotropize. For the electrons, at least, this assumption is consistent with observations (e.g., Bame et al. [1986]). Using this assumption, the divergence of the pressure tensor can be replaced with the gradient of a scalar 
pressure, which is simply the sum of the electron and lon pressures, $P=n_{e} k T_{e}+n_{1} k T_{1}$ where $k$ is Boltzman's constant. The internal energy of an 1sotropic plasma 1s, then, $U=(3 / 2)$. We further assume that the plasma remains electrically neutral, $\rho_{c}=0$, and that the heat flux is negligible within the tall, $q=0$. Finally, we assume that the plasma flows everywhere antiparallel to the $X-$ axis, $\nabla=v_{X} \hat{X}$, in accordance with global MHD simulations by Fedder et al. [1986] which show that there is only very slight deflection of the plasma flow throughout the global cometary interaction. This assumption cannot be precisely true for streamlines which are diverted about the conductive 1onosphere, however, such diversion only slightly increases the streamlines' path lengths compared to the distance back to ICE, and some such assumption is required to make the equations tractable with the 11mited ICE data avallable. In the MHD hydromagnet1c $\lim 1 \mathrm{t} \tilde{\mathrm{E}}=-\tilde{\mathrm{v}} \times \tilde{\mathrm{B}}$, which under the above assumptions becomes $\tilde{E}=-v_{X} B_{Y}, \hat{z}^{-}$. Combining equations 4.4-4.6 with equations $4.1-4.3$, Inserting these simplifications, and solving for the steady state solutions ylelds the greatly simplified MHD equations

$$
\begin{aligned}
& \frac{\partial}{\partial X}\left(\rho v_{X}\right)=s^{*} \\
& \frac{\partial}{\partial X}\left(\rho v_{X}^{2}+P\right)=-B_{Y}-J_{Z}-
\end{aligned}
$$




$$
\begin{aligned}
& \frac{\partial}{\partial Y^{\circ}}(P)=B_{X^{\prime}} Z^{-} \\
& \frac{\partial}{\partial X}\left(\frac{1}{2} \rho v_{X}^{3}+\frac{5}{2} P v_{X}\right)=-X^{B} Y^{-}-J_{Z^{-}}
\end{aligned}
$$

where equation 4.7 explicitly describes the addition of mass to the plasma flow, equations $4.8 \mathrm{a}$ and 4.9 describe the tallward acceleration of the plasma, and equation $4.8 \mathrm{~b}$ describes the pressure balance across the tall.

Since $J_{Z^{-}}$is simply the derivative of ${ }^{B} X$ with respect to $Y^{*}$, as given in equation 4.6 , equation $4.8 \mathrm{~b}$ can be rewritten in the more common pressure balance form

$$
\mathrm{nk}\left(\mathrm{T}_{1}+\mathrm{T}_{\mathrm{e}}\right)+\frac{\mathrm{B}_{\mathrm{x}}{ }^{2}}{2 \mu_{0}}=\mathrm{P}_{\mathrm{T}}
$$

for singly lonized lons where $\mathrm{P}_{\mathrm{T}}$ 1s the total pressure across the tail, a constant at any given $X$ distance downtail. In the pressure balance condition described by equation 4.10 , all quantities on the left-hand side are measured throughout the ICE tall traversai except the average ion temperature, $T_{1}$. In order to calculate $T_{1}$ as a function of location across the tall, we must first determine $P_{T}$ at the downtall distance of the ICE traversal. S1scoe et al. [1986] argued that the tall pressure is approximately the stagnation 
pressure of the solar wind since the plasma pressure at the comet essentlally stands off the solar wind flow. Here, we Improve this argument to Include the effects of the residual plasma flow within the near comet environs.

At any near comet location the local plasma and field pressures must stand off the difference between the upstream and the local dynamic pressures in order to be in equilibrium. Since the electromagnetic term in equation $4.8 \mathrm{a}$ is the $X$ derivative of some (possibly unknown) function, it can thereby be absorbed into the $\mathrm{X}$ derivative, along with the plasma pressure, on the left-hand side of the equation. Integrating this equation along a streamline (fixed $\mathrm{Y}^{\circ}$ and $\mathrm{Z}^{\circ}$ values) ylelds

$$
P_{c}=\rho_{s w} v_{s w}^{2}-\rho_{c} v_{c}^{2}
$$

where $P_{C}$ represents an 1sotropic pressure due to both the plasma and fleld, and $\rho_{c}$ and $v_{c}$ are the mass density and velocity assuming that all three quantities are taken at a single location within the comet. The upstream solar wind plasma and field pressures are only a few percent of the solar wind ram pressure $\left(\rho_{s w} v_{s w}^{2}\right)$, and are ignored in this equation.

Equation 4.11 holds for the pressure on the external surface of the magnetotall so that $P_{C}=P_{T}$ and all quantities in equations 4.10 and 4.11 are measured except for $T_{1}$. The solar wind electron density just prior to the ICE fly-through was $\sim 5 / \mathrm{cm}^{3}$, and just after was $\sim 7 / \mathrm{cm}^{3}$ so that we use $\mathrm{n}_{\mathrm{sw}}=6 \pm 1 \times 10^{5} / \mathrm{m}^{3}$. The 
electron density at the magnetotall boundary, at the location of the ICE entry and exit, is $n_{c}=75 \pm 25 \times 10^{6} / \mathrm{m}^{3}$, and we assume that the typlcal pick-up ion has a mass of 18 amu (water group) as Indicated by the results of OgIlvie et al. [1986], and Zwlckl et al. [1986]. The solar wind veloclty extrapolated from the top panel of Figure 4.2 is $v_{\mathrm{sw}}=460 \pm 25 \mathrm{~km} / \mathrm{s}$. The plasma velocity at the tall boundary is less than the Los Alamos measurable minlmum value of 30 $\mathrm{km} / \mathrm{s}$, and for the purposes of this calculation we use $v_{c}=20 \pm 10 \mathrm{~km} / \mathrm{s}$ which w1ll be shown to be a reasonable value later In this section. Inserting a11 these values into equation 4.11 , the most probable total tall pressure at the ICE crossing is $P_{T}=1.2 \times$ $10^{-9} \mathrm{~N} / \mathrm{m}^{2}$ with an upper bound of $2.6 \times 10^{-9}$, and a negative (unphysical) lower bound. The value used by siscoe et al. [1986] was $\sim 2.5 \times 10^{-9}$, a factor of two greater than the most probable value calculated here.

The measured plasma electron pressure is displayed in the top panel of Figure 4.5. The pressure maximizes in the central region of the current sheet, and is relatively constant throughout the two tall lobes. The cross tall component of the magnetic fleld pressure, $B_{X}^{2} / 2 \mu_{0}$, is displayed in the third panel of the figure. The fleld pressure varies in a manner generally opposite to the plasma electron pressure minimizing in the cross tail current sheet and maximizing in the portions of the lobes lmmediately adjacent to this sheet. Inserting $P_{T}=1.2 \times 10^{-9} \mathrm{~N} / \mathrm{m}^{2}$ 1nto equation 4.10 , and using these two measured pressure varlations, we calculate the variation of $T_{1}$ across the tall traversal. The second panel of 

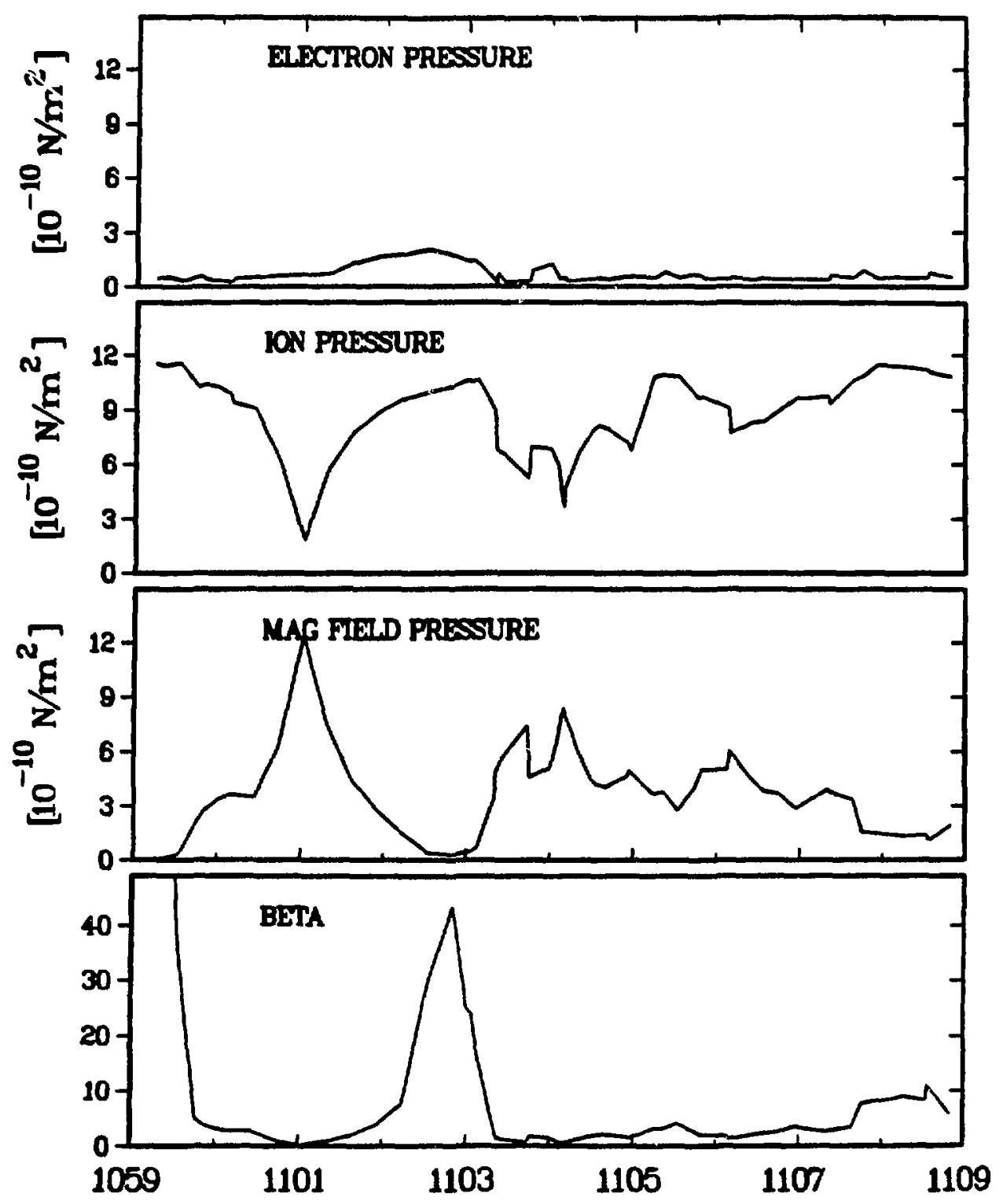

Fig. 4.5. Pressure variations across the magnetotail. The first and third panels show the plasma electron and magnetic fleld pressures while the second panel displays the plasma ion pressure profile infirred from equation 4.10. The total plasma beta is shown in the bot:nm panel. Note that the lobes (low beta regions) and current sheet (central high beta region) are easily discernible, as are inbound and outbound tall crossings at $\sim 10: 59: 40$ and $\sim 11: 07: 40$. 
Figure 4.5 displays this calculated lon pressure on the same scale as the electron plasma and magnetic fleld pressures. Note that the Ion pressure is apprectably larger than the electron pressure throughout the entire tall traversal, and accounts for the majority of the plasma pressure in the balance condition.

The bottom panel of Figure 4.5 shows the calculated beta of the plasma. In this panel the tall lobe entry at $\sim 10: 59: 40$ and exit at 11:07:40 are easily discernible, as 1s the crossing of the center of the current sheet at $\sim 11: 02: 50$. The plasma beta varles rather smoothly through the current sheet traversal maximizing at the center, while sheet entry and exit times can be somewhat arbitrarily defined at $\sim 11: 01: 20$ and $\sim 11: 03: 20$, respectively. The beta in the G-2 tall lobes is generally in the range from $1-4$ throughout the entire magnetotall traversal at $7800 \mathrm{~km}$ downtall, and rises to $\sim 40$ in the center of the current sheet. This situation is quite different from the deep Venus magnetotall where the lobe and current sheet betas are $\sim 10^{-1}$ and $\sim 10^{1}$ respectively [McComas et al., 1986a]; however, it seems likely that farther downstream in the G-Z magnetotall the beta may be reduced due to reacceleration of the plasma and the consequent decrease in the plasma density.

The derived Ion pressure profile in the second panel of Figure 4.5 is converted Into an equivalent temperature profile assuming the equation of state $P=n k T$ as above, and 18 plotted in the top panel of Figure 4.6. The 1on temperature is quite constant in the current sheet, with a value of $\sim 1.2 \times 10^{5} \mathrm{~K}$. Just outside the boundarles of the tall it is also relatively constant with a value of $1-1.5 \times$ 


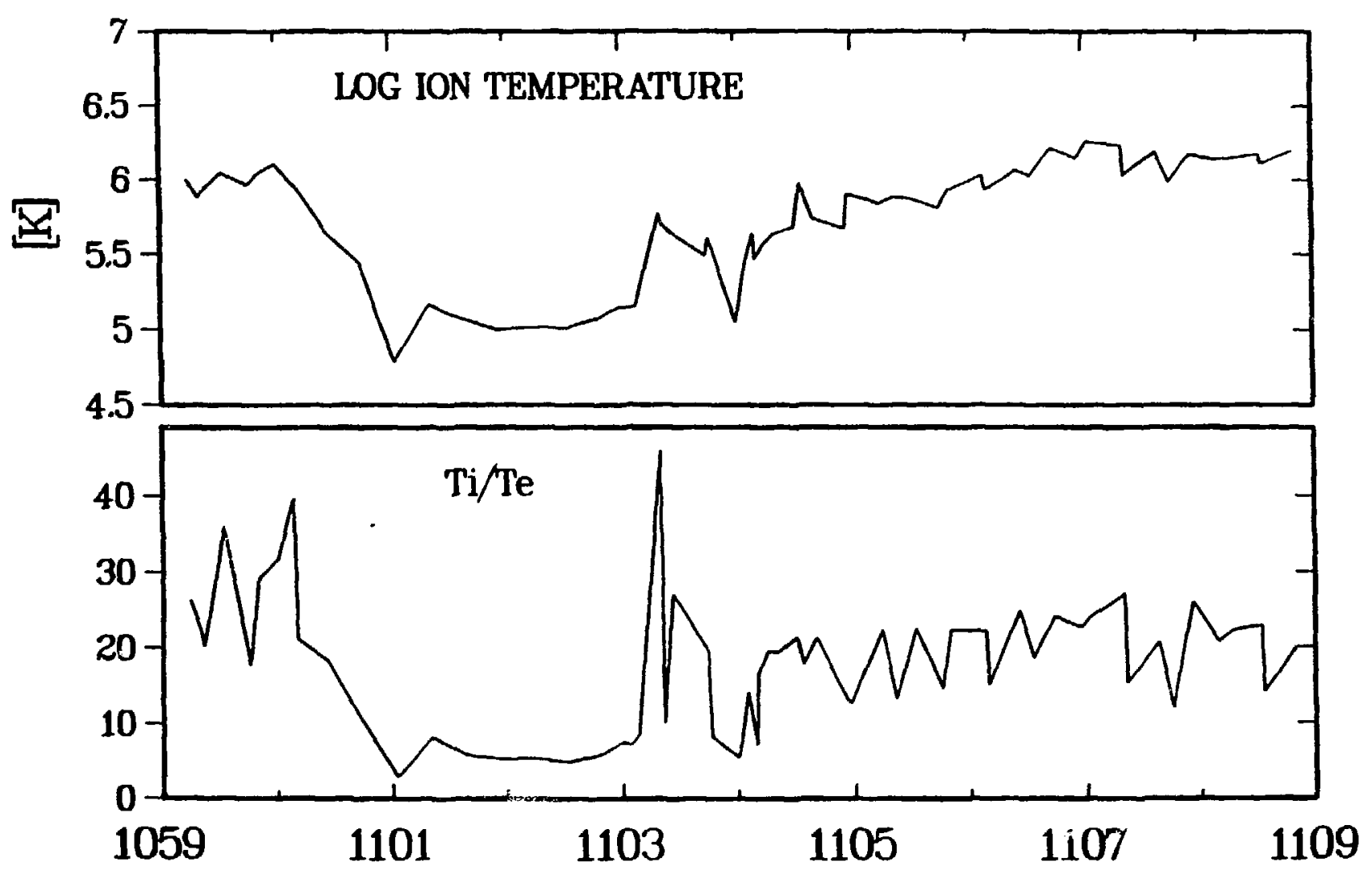

Fig. 4.6. Ion temperature derived from the ion pressure profile displayed in the previous figure. The bottom panel shows the ratio of this dervied temperature to the measured electron temperature. This ratio is relatively constant in the lobes and current sheet with values of approximately 20 and 6.5 , respectively. 
$10^{6} \mathrm{~K}$. Across the lobes, the Ion temperature varles relatively smoothly between these two values. The bottom panel of Figure 4.6 shows the ratio of $T_{1} / T_{e}$ across the tall. This ratio is also relatively constant within the current sheet with a value of $\sim 6.5$, Indicating that neglecting the electron pressure term in the current sheet would have caused about a $15 \%$ error in the calculation of the current sheet ton properties. The temperature ratio is also relatively constant, with a value of $\sim 20$, throughout most of the rest of the ten minute tail traversal. Such a constant ratio is rather surprising since the electron temperature is presumably determined by cooling rates from the typical photolonization energy of $\mathrm{H}_{2} \mathrm{O}$ [Zwlckl et al., 1986], while the ton temperature is a function of the flow speed in the average ion plck-up region, as we now describe.

The pick-up ion temperature is not just interesting in its own right, but also contalns significant information about the plasma properties at the average location where the cometary lons are picked-up. As pointed out by McComas et al. [1986b] and S1scoe et a1. [1986], the average Ion temperature in an Induced magnetotall is Indicative of the velocity of the bulk plasma flow at the average pick-up location. When a molecule is lonized, either by photolonization or charge exchange, the newly formed ton is pickedup by the motional electric fleld of the flowing, magnetized plasma. The relative motion of the new fon and the flow is essentially the plasma flow speed itself and, therefore, the lon is picked-up with an Initial energy equal to the translational energy of the ion in 
the plasma rest frame. Eventually, one degree of freedom of translational energy, whlch has been picked-up into some comblnation of parallel and perpendicular energies, is isotropized into three degrees of freedom of isotropic thermal energy

$$
\frac{1}{2} \mathrm{~m}_{1} \mathrm{v}_{\mathrm{pu}}^{2}=\frac{3}{2} \mathrm{kT}_{\mathrm{pu}}
$$

where $m_{1}$ is the mass of a pick-up ion $(18 \mathrm{amu})$ and $v_{\mathrm{pu}}$ is the plasma flow velocity at the pick-up location.

In the previous study of MHD equilibrlum within the G-Z magnetotail [S1scoe et al., 1986] the Ion temperature was assumed to be independent of the downtail, $X$, distance. Using this assumption, an Inferred ion temperature profile (such as is displayed in the top panel of Figure 4.6) can be directly converted into an inferred upstream pick-up velocity profile. In this study we improve the derivation of the relationship between the upstream conditions along each streamline, at the average location upstream from ICE where an Ion 1s picked-up, and at the ICE traversal, by retaining independent variations of the lon plasma temperature and density with $x$.

Since $v_{\mathrm{pu}}$ is a measure of the plasma velocity at the average location where an Ion is picked-up, half of the mass picked-up along each streamline is picked-up sunward of the average location and half tallward of this location. Thus, for constant cross-sention stream tubes $\left(\nabla=v_{X} \hat{X}\right)$, the number flux of plcked-up 1ons, nv, at 
the average plck-up location is only one half of that measured at: ICE,

$$
n_{p u} v_{p u}=\frac{1}{2} n_{I C E} v_{I C E}
$$

where ill four quantities are defined along a streamline, and $\mathbf{n}_{I C E}$ and $V_{I C E}$ are additionally defined along the ICE traversal. The average pick-up properties defined in this way are representative of averages over locations sunward from ICE, but are probably very close to the actual average locations along each streamline since the vast majority of the tall mass loading must occur sunward of ICE. The addition of mass tallward from these average plck-up locations acts to slow the plasma flow further and Increase the plasma denstty beyond the values at the average pick-up location.

The $X$-components of the momentum equation ( $4.8 \mathrm{a})$ and the energy equation ( 4.9 ) can be integrated along each streamline between the average plck-up locations upstream, $X_{p u}$, and the ICE traversal crossing distance, $X_{I C E}$, to yleld

$$
\begin{aligned}
& n_{I C E}\left[m_{1} v_{I C E}^{2}+k T_{I C E}\right]-n_{p u}\left[m_{1} v_{p u}^{2}+k T_{p u}\right]= \\
& \int_{x_{p u}}^{X_{I C E}}-B_{Y}-J_{Z^{-}} d X
\end{aligned}
$$

and

$$
n_{I C E} v_{I C E}\left[m_{1} v_{I C E}^{2}+5 k T_{I C E}\right]-n_{p u} v_{p u}\left[m_{1} v_{p u}^{2}+5 k T_{p u}\right]=
$$




$$
2 \int_{X_{p u}}^{X_{I C E}}-v_{X} B_{Y} \cdot J_{Z}+d X
$$

where the electron component of the pressure has been neglected since it is much smaller than the lon component as demonstrated in Figure 4.5. Comblning these equations with the relation between the pick-up velocity and temperature (equation 4.12), and the relation between the average pick-up location and ICE traversal number fluxes (equation 4.13) provides a set of four independent equations which relate the plasma and field properties upstream at the average pickup location, and at the ICE traversal. The plasma density, $\mathrm{n}_{\text {ICE }}$, is measured at each location along the ICE trajectory, $X=X_{I C E}$, while the ion temperature $T_{I C E}$ has been Inferred at each location. The remaining unknowns in this set of equations are: $v_{I C E}, v_{p u}, T_{p u}$, $n_{\mathrm{pu}}, \mathrm{X}_{\mathrm{pu}}$, and the electromagnet1c $(\tilde{J} X \tilde{B})_{X}$ force and plasma velocity, $v_{X}$, as functions of $X$.

In order to close this set of equations and derive the condition for the $\mathrm{X}$-directed force balance, the electromagnetic term, $\mathrm{B}_{\mathrm{Y}^{-}} \mathrm{J}_{\mathrm{Z}^{-}}$, as a function of $\mathrm{X}$ must first be determined. The cross tall current density, $\mathrm{J}_{\mathrm{Z}^{-}}$, is defined in equation 4.6 while the field variations across the tall along the ICE trajectory are displayed in Figure 4.4. The spatial derivative of $\partial \mathrm{B}_{\mathrm{X}} / \partial \mathrm{Y}^{-}$is extremely sensitive to the fine scale $B_{X}$ variations shown in the third panel of this figure. Such variations are not indicative of the global, average configuration of field draping within the tail, but rather probably represent smaller scale structures embedded within the tail, as discussed in the first section, and time 
dependent phenomena, such as waves and instabilities. In order to determine the more global, average configuration it is first necessary to smooth the high resolution $B_{X}$ data. We have examined the results of a number of smoothing intervals, and found that a 60 second running average best removes the high frequency fluctuations without suppressing large scale varlations across the tall. From this smoothed field dato the cross tall current density, $J_{Z^{-}}$, has been calculated.

Figure 4.7 displays the $X$-component of the electromagnetic $\tilde{J} \times \tilde{B}$ force, $\mathrm{B}_{\mathrm{Y}^{-}} \mathrm{J}_{\mathrm{Z}^{-}}$. Throughout the current sheet this force is negative (directed tafiward), and acts to accelerate the picked-up plasma and restraighten the kinked field configuration. In the lobes, the $\mathrm{X}$ component of $\tilde{\mathrm{J} x} \tilde{\mathrm{B}}$ is generally positive due to the fact that the fleld is st 111 being stretched and draped into a tall configuration through this region. Clearly there is also still a substantial amount of fine structure in Figure 4.7, and plasma properties derived from tt will contain substantial fluctuations superimposed on the average variations across the tail. In particular, the negative $(\tilde{\mathrm{JxB}})_{\mathrm{X}}$ region at $\sim 11: 06$ may be Indicative of a re-encounter with the current sheet, or may be due to an embedded structure or dynamic effects. This variation is left in since it is large scale; however, the meaningfulness of derived results from this short Interval for the general tall structure is questionable.

The variation shown in Figure 4.7 describes the $\bar{J} \times \bar{B}$ force as measured along the ICE traversal which is the only location where it 1s known. In equations 4.14 and 4.15 , however, the right-hand sides 


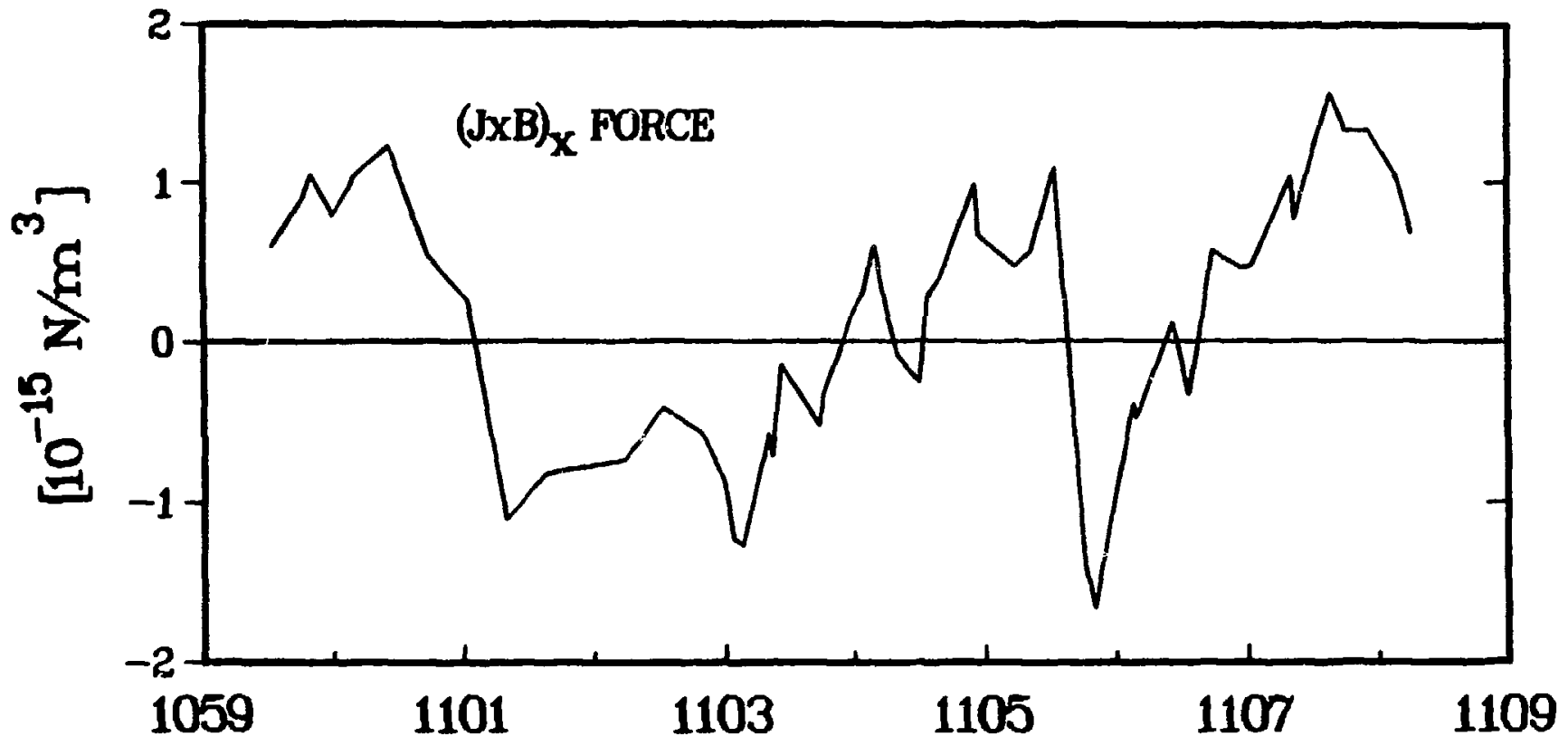

Fig. 4.7. X-component of the electromagnetic $\mathrm{J} \times \mathrm{B}$ force across the tail. The force is negative (tailward) throughout the current sheet, serving to reaccelerate the most highly mass loaded portion of the flow. In the lobes the force is generally positive (sunward) indicating that the field in these regions is still being stretched into a tall-1ike configuration. 
are Integrals over $X$ between $x_{p u}$ and $x_{I C E}$. In order to make these equations tractable with the limited ICE data set we make two simplifying approximations. First, we assume that $B_{Y}-J_{Z}$, can be approximated with a single value over the limited range from $x_{\text {pu }}$ to $\mathrm{X}_{\mathrm{ICE}}$. Upstream in the center of the current sheet the $\tilde{J} \times \tilde{B}$ force $1 \mathrm{~s}$ probably simflar to its value at ICE since the effects of field Iine tension and mass addicion are competing in the current sheet, and since the field configuration is draped all the way up to the nucleus; so the field configuration and forces should be only slowly varying functions of distance. In the lobes, on the other hand, both the mass addition and the $j \times \tilde{B}$ force act to slow the plasma substantially and Increase its density over that upstream while the field continues to stretch about the obstacle and fold into the ta11. The $\tilde{J} \times \tilde{B}$ force should, therefore, decrease with distance upstream, and be approximately zero sunward of the flaring tafl boundary. In order to model this complicated variation as a function of location across the tall as a function of a simple varying value of $B_{Y^{-}} J_{Z^{-}}$, we assume that this value follows the variation measured by $I C E$, as shown in Figure 4.7 , scaled by a factor which varies from one in the center of the current sheet to zero at the edges of the tall. This is by far the most suspect assumption in this derivation, however, its appropriateness w111 be further supported by Independent measurements of the derived plasma velocity at ICE.

The other assumption needed to close the set of equations deals with the lntegral of the plasma velocity with downtall distance. We 
assume that the Integral of $v_{X}$ over $X$ is approximately 1 ts average value times the $X$ distance: $\frac{1}{2}\left(v_{p u}+v_{I C E}\right)\left(X_{I C E}-X_{p u}\right)$. In the upstream region of the current sheet the average plck-up location is probably very close to the nucleus since the neutral denstty is largest there and drops off rapidly with distance. Upstream from the lobes the situation is somewhat less clear, but the average pick-up location is st111 probably near the location of maximum neutral density, $X=0$. Therefore, we use $\left(X_{I C E}-X_{p u}\right)=-7800 \mathrm{~km}$ across the tall.

Including these approximations, equations 4.14 and 4.15 can be combined with equations 4.12 and 4.13 to solve for the four unknowns: $v_{I C E}, v_{p u}, T_{p u}$, and $n_{p u}$ at each data point along the ICE trafectory. This set of equations is somewhat complicated, being quadratic in both $v_{p u}$ and $v_{I C E}$. Therefore, instead of solving them analytically, we choose to solve the set of equations with an iterative computer technique.

The results of this calculation are displayed in Figure 4.8 . The top panel shows the derived plasma velocity variation across the tall along the ICE trajectory. Through the current sheet the tallward velocity is somewhat variable with an average value $\sim 20$ $\mathrm{km} / \mathrm{sec}$. In the lobes the velocity is more variable, having an average value somewhere from -20 to $-30 \mathrm{~km} / \mathrm{sec}$, wh1ch 1ncreases slightly toward the sides of the tall. The large fluctuations across the plot are probably not iridicative of the average, global structure, but rather embedded fine structures and time dependent effects. St111, the general result that the velocity profile at the 


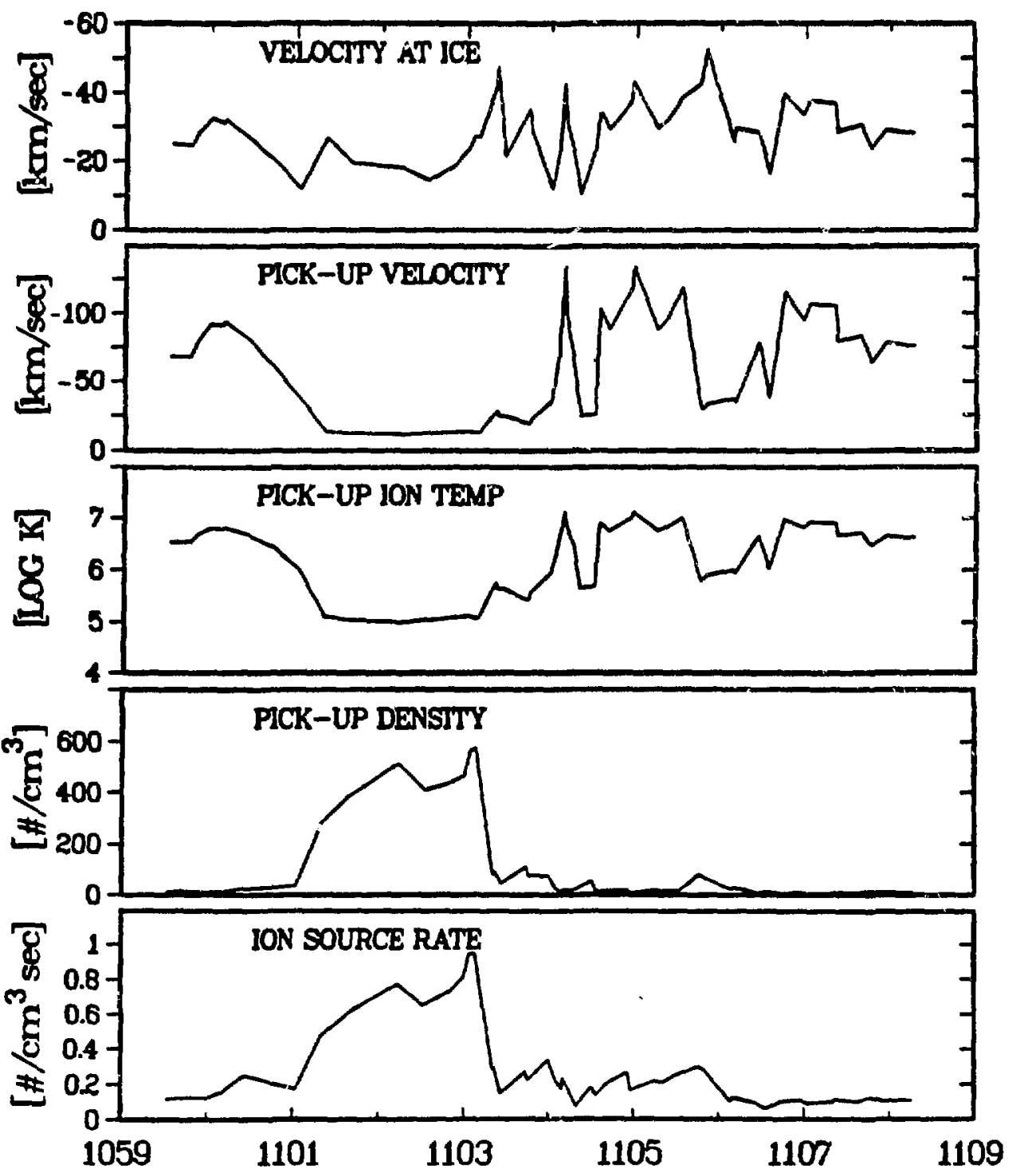

Fig. 4.8. Results of the observation based MHD calculation of the flow velocity at ICE and plasma properties upstream at the average pick-up location. The top two panels display the velocity profiles at ICE and the average pick-up location, and indicate that the velocity shear responsible for creating the draped tail configuration occurs upstream from the ICE tail traversal. The third and fourth panels show the average pick-up temperature and density, while the fifth panel displays the calculated ion source rate averaged over $7800 \mathrm{~km}$ from the average upstream pick-up location back to ICE. Sharp gradients between the current sheet and tail lobes in these various parameters indicate that the current sheet is downstream from a "prime mass loading region" which has a substantially enhanced source rate. 
ICE traversal distance is nearly flat is well resolved by our calculation.

The result that the plasma velocity along the ICE tall traversal is nearly constant can be independently verified with the ICE magnetic field measurements. In a steady state solution of Faraday's Induction Law, $\nabla \times \tilde{E}=0$. In the MHD hydromagnetic $11 \mathrm{mit}$ with $B_{Z^{-}}=0$, this corresponds to the statement that $v_{X^{-}} B_{Y^{-}}=$ constant. The fourth panel of Figure 4.4 shows that $\mathrm{B}_{\mathrm{Y}}$, is on average quite constant across the ten minute interval. The plasma velocity must also, therefore, be reasonably constant as derfved here.

As a further check, the observational lower 1imit for bulk velocity determinations at $G-Z$ with the Los Alamos electron analyzer is $\sim 30 \mathrm{~km} / \mathrm{s}$. Plasma electron data for the crossing indicate that a velocity in excess of this value was not consistently achieved for $\sim 9$ and $\sim 6$ minutes beyond the inbound and outbound edges of the magnetota11, respectively. The calculated plasma velocity displayed In the top panel of Figure $4.8 \mathrm{is,} \mathrm{therefore,} \mathrm{generally} \mathrm{in} \mathrm{excellent}$ agreement with the lack of direct velocity observations through the tall region. The variation of velocity across the tall is sensitive to the assumptions made above in general, and the treatment of the electromagnetic force term in particular. The good agreement between the derlved velocity and the ICE field and plasma observations supports the general validity of those assumptions.

The plasma parameters derived for the average upstream pick-up location, $X \approx 0$, are displayed in panels 2-4 of Figure 4.8. Panels 
2 and 3 show the calculated average lon pick-up velocity and equivalent ion temperature, wh1ch are related by equation 4.12 . The velocity and temperature are extremely constant through the current sheet with values of $\sim-12 \mathrm{~km} / \mathrm{sec}$ and $\sim 1 \times 10^{5} \mathrm{~K}$, respectively. These values become highly variable and rise outside the current sheet, in the lobes, to average values of $\sim-75 \mathrm{~km} / \mathrm{sec}$ and $\sim 4 \times 10^{6} \mathrm{x}$ near the edges of the tall. The difference between the velocitles upstream from the lobes and current sheet is fundamentally responsible for forming the magnetotall configuration observed at ICE since the large velocity shear drapes the solar wind's embedded magnetic field. In the Discussion section we will examine the 1mplications of the derived velocity shear upstream and the nearly constant velocity across the tall at ICE, and discuss some of the more detalled aspects of the tail formation process.

The derived ion density at the average upstream pick-up locations is plotted in panel 4. The region upstream from the current sheet has a density of $\sim 200-60010 n s / \mathrm{cm}^{3}$ wh1le the regions upstream from the lobes have decreasing densitles away from the current sheet, and an average value across the lobes of only $\sim 20$ $1 \mathrm{~cm}^{3}$. Comparing this panel with the measured density along the ICE trajectory (top panel of Figure 4.4) Indicates that the gradient in number density across the tall is much larger near the comet nucleus than it is back in the tail, and that the region of enhanced density also has sharper boundartes upstream near the nucleus.

From the derived density and velocity profiles at ICE and upstream, the average ion source rate, $s^{*} / m_{1}$, between these two 
locations, can be readily found. The time independent continuity equation (4.7) evaluated between ICE and the average pick-up location is

$$
\frac{n_{I C E} v_{I C E}-n_{p u} v_{p u}}{x_{I C E}-x_{p u}}=s^{*} / m_{1}
$$

and the results of this calculation are displayed in the bottom panel of Figure 4.8. It must be remembered that this is an average over $7800 \mathrm{~km}$ and, therefore, the source rate is probably much greater within the range close to the nucleus, $0 \gtrsim x \gtrsim-1000 \mathrm{~km}$. Still, the fon source rate is clearly much greater in the region upstream from the current sheet than from the lobes. The "prime mass loading" region discovered in this study is only $1500 \mathrm{~km}$ across in $\mathrm{Y}^{\top}$, and Implications of this small region of high density, slowly flowing plasma where a substantial portion of the mass loading occurs, are examined in more detall in the Discussion section.

\subsection{Cometary Mass Transport}

The mere existence of comet talls, and earthbound obseryations which often show tallward propagating features, Indicate that substantial numbers of cometary lons are transported down the high density Ion tall or magnetotall current sheet. At venus, the majority of the lost planetary lons are apparently transported down the magnetotail (see calculations by McComas et al. [1986a], and 
reference therein). At comets, the mass loading region is much more extended than at Venus since the cometary neutral atmosphere 18 not gravitationally bound, and a far smaller fraction of the plcked-up ions may be involved in draping the magnetic fleld into a magnetota11.

Calculations by Mendis et al. [1986], which combine the model of Schmidt and Wegmann [1982] and typlcal plasma and fleld values observed during the ICE encounter, yleld a cometary loss rate of $4.0 \times 10^{28}$ molecules/s. Direct measurements of the bow wave crossing locations by Jones et al. [1986] and Ind1rect measurements by Fuseller et al. [1986] yleld loss rates of 2.3-2.5 and $3 \times 10^{28}$, respectively. Within the errors of the varfous techniques, these three values are all consistent.

In this study we make a more detalled examination of where these cometary lons are plcked-up by the solar wind flow by examining the lon transport rate (number flux) from the direct ICE observations. The fon number flux, $\mathrm{nv}$, is a direct measure of the number of lons passing downtall per untt area and time. of course neutrals are not measured in the number flux, but all neutrals which are lonfzed sunward of the ICE traversal plane are carrled tallward through the plane as lons, and are counted in the number flux. These include all neutrals with an Inftial sunward velocity and those with an initial tallward velocity that are lonized sunward from the ICE traversal. Therefore, more than half of the neutral cometary efflux w111 pass through this plane as lons added to the flow. 
The rate at which lons are lost down the Glacobini-zinner magnetotall can be calculated by integrating the particle number flux over the tall cross-section. The cross-sectional tall geometry at the downtall distance of the ICE traversal is shown in Figure 4.3. For this calculation we use the measured density distribution shown in the top panel of Figure 4.4 and a constant downtall velocity of $-20 \mathrm{~km} / \mathrm{s}$, as discussed in the previous section. Combining these data, and integrating $1 \mathrm{n} 10^{4} \mathrm{~km}$ long strips parallel to the $Z^{\circ}$-axis we find that the total number of particles flowing down the magnetota11 is $\sim 2.6 \times 10^{26}$ molecules/s.

A number flux of $\sim 2.6 \times 10^{26 / s}$ lost down the magnetota11 represents only $\sim 1 \%$ of the total cometary loss rate quoted above. Such a result is not unreasonable in light of the extremely extended nature of the neutral atmosphere and, therefore, mass addition region. It does, however, bring to light the question of where the major mass plck-up and transport occurs.

To address this question we calculate the particle loss through a cylinder which encompasses the extended mass loading region, as drawn in Figure 4.9 The axis of the cylinder is parallel to the $X-$ axis and the unperturbed solar wind approaches the comet along the -X-direction. The cylinder has a radius of $\sim 2.4 \times 10^{5} \mathrm{~km}$ which extends out to the region along the flanks of the comet where no significant slowing assoclated with lon pick-up coyld be detected. The upper surface of the cyl1nder 11 es well upstream of the comet. We assume that the external solar wind flow was constant during the Inbound (outbound) portion of the ICE traversal with a number flux 


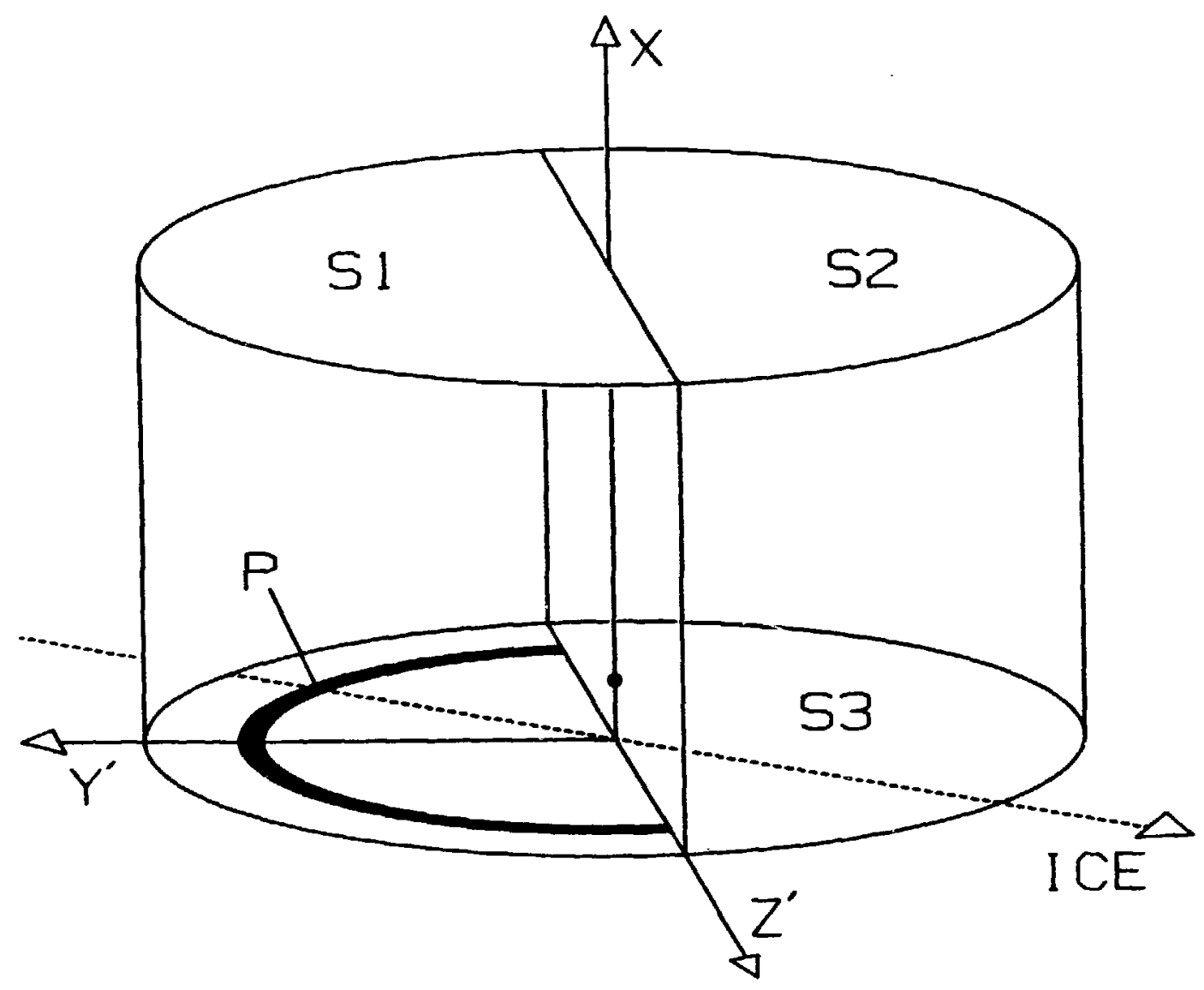

Fig. 4.9. Schematic diagram of the geometry used for calculating the cometary neutral gas efflux. The axts of the cylinder is aligned with the solar wind flow, and is wide enough to encompass the entire cometary mass loading region back to the plane of the ICE traversal. The difference in the number of particles crossing the top surface, $S 1+S 2$, and the bottom surface, $S 3$, must have been added to the flow within the cylinder. The number of particles crossing 53 is calculated by assuming uniformity within half circular annuli along the ICE trajectory. 
$n_{1} v_{1}\left(n_{2} v_{2}\right)$ passing through the surface $S 1$ (S2), and equal to that measured just outside the cylinder along the ICE trajectory. The ICE spacecraft traverses the comet in the plane of the bottom surface, 53 , of the cylinder. We assume that the plasma conditions are approximately cylindrically symmetric, as Indicated by MHD models of G-Z at this downtall distance [Fedder et al., 1986], and In particular, that the observed properties constitute average values for half circular annull at each point, 9 , along the trajectory. By 1ntegrating the plasma properties over these annul1, the total number of particles crossing surface $\$ 3$ is determined. If the comet is ejecting molecules at an approximately steady rate, $Q$, then the number of cometary particles picked-up by the flow, sunward of the ICE traversal, is

$$
Q=\sum_{a} n(t) v(t) A_{a}-\left(n_{1} v_{1} s 1+n_{2} v_{2} s 2\right)
$$

where $n(t)$ and $v(t)$ are the observed density and tallward velocity of the plasma, and $A_{a} 1 s$ the annulus area at each point $P$ along the trajectory.

The smoothed electron density profile for the entire G-Z crossing is shown on a logarithmle scale in the top panel of Figure 4.10. Ground based observations of comets integrate such density profiles along a line of sight, and it is not surprising that only the magnetotail and visible coma stand out in such observations. The fact that the density is large, however, does not 

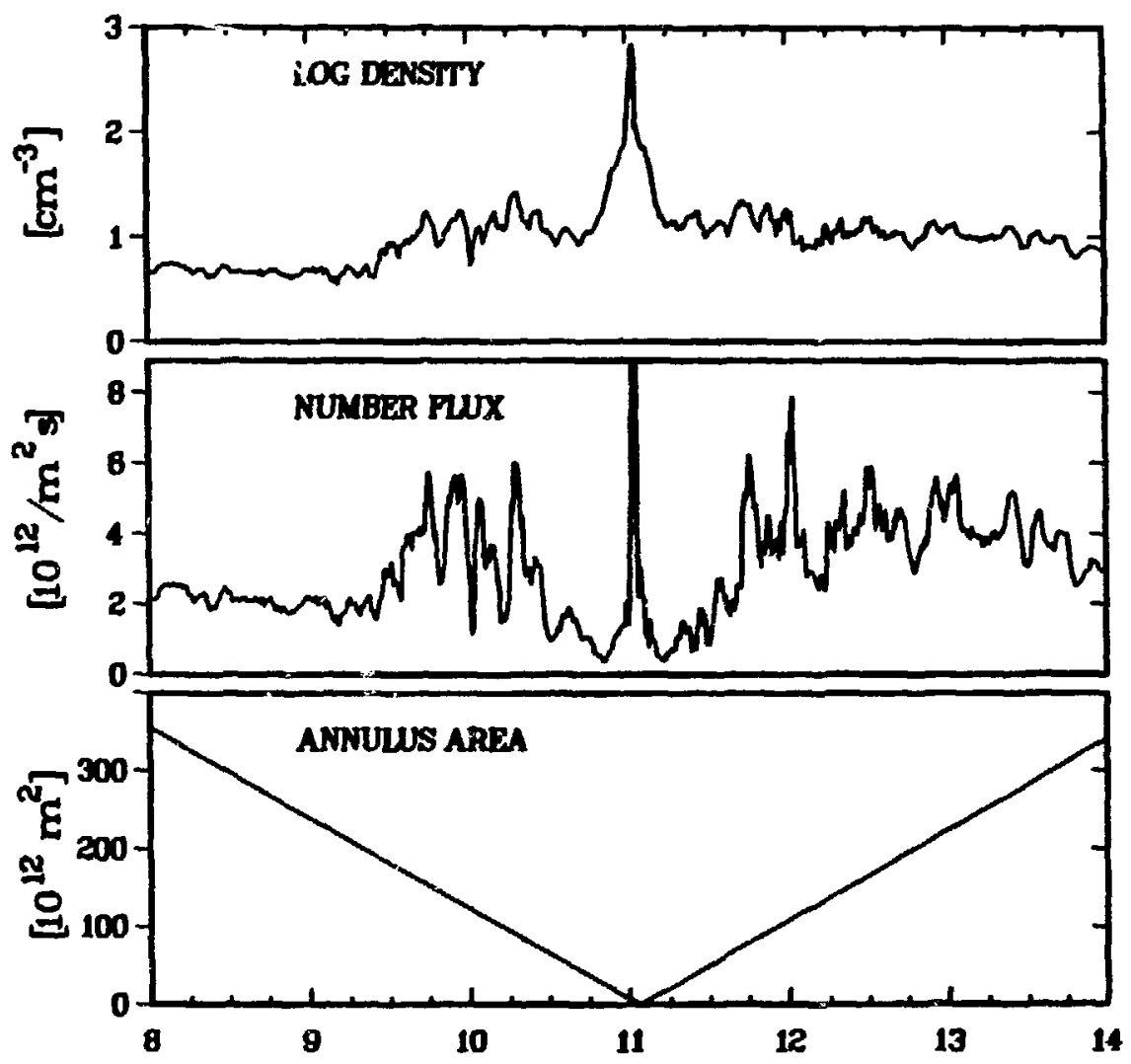

Fig. 4.10. Parameters used in the calculation of the cometary efflux. The plasma density is clearly maximized in the magnetotall (narrow feature at $\sim 11: 00$ ) and surrounding coma, which accounts for the high visibility of these regions. The middle panel displays the number flux, $n$, which also maximizes in the magnetotall current sheet, rising off the top of the plot to a value of $14 \times 10^{12} / \mathrm{m}^{2} \mathrm{~s}$. Data in both of the top two panels have been smoothed with a nine point running average except in the narrow tail region. The majority of the cometary particles are apparently lost in regions far from the comet nucleus, largely due to the increase in effective transport area with distance as depicted in the bottom panel. 
imply that the particle flux in these regions is large since the flow Is also slow there,

The calculated number flux of particles is shown in the middle panel of Figure 4.10. The flux is clearly highly variable but generally maximizes in the transition region and outer magnetosheath, far from the magnetotall 1tself. In fact, other than within the narrow tall current sheet, the flux was smaller in the magnetotall than in the surrounding solar wind. The portions of the profile from 8:00 to $\sim 8: 45$ and $\sim 13: 00$ to $14: 00$ are essent1ally unperturbed solar wind, as evidenced by the velocity profiles displayed in Figure 4.2 , and are used to fix the upstream values for the Inbound and outbound portions of the crossing. These values are $\sim 2$ and $\sim 4 \times 10^{12} / \mathrm{m}^{2} \mathrm{~s}$, respectively, and are used to determine the flow through surfaces $S 1$ and $S 2$ in Figure 4.9 and equation 4.17 . Velocity vartations provide a much more sensitive indicator of Ion plck-up than number density variations because. cometary pick-up lons are much heavier than the average solar wind lons (protons). The number density reflects only increases in the tocal number of added lons, and for example, a $1 \%$ addition of pick-up tons of average mass 20 1ncreases the number density by about the relative Instrumental resolution of the Los Alamos plasma analyzer, $1 \%$. However, the mass density is increased by $\sim 20 \%$, and the flow speed should be redused to about $80 \%$ of 1 ts unperturbed valie, providing an easily resolvable signature.

The bottom panel of Figure 4.10 displays the annulus area per 24 second plasma data sample. Th1s value was simply calculated from 
the location of the spacecraft as a function of time and the constant crossing velocity in the $Y^{\circ}-Z^{\circ}$ plane. Since it is the product of the bottom two panels which gives the rate of particle loss, the cuter portions of the crossing are clearly the dominant regions for particle transport.

Using the values of 2 and $4 \times 10^{12} / \mathrm{m}^{2}$ s for the upstream solar wind, as given above, we find a solar wind particle flux of $2.11 \times 10^{29} / \mathrm{s}$ entering the top of the cylinder. Integrating the loss rate of particles through surface $s 3$, we find a flux of $2.36 \times 10^{29} / \mathrm{s}$ exiting the bottom surface of the cylinder. Finally, using equation 17 to take the difference of these two large numbers, we find that the number of cometary lons plcked-up by the solar wind flow, sunward of the ICE traversal, $1 \mathrm{~s} 2.5 \pm 1.5 \times 10^{28} / \mathrm{s}$. The upper and lower bounds are arrived at by assuming number fluxes of 1.8 and $3.8 \times 10^{12} / \mathrm{m}^{2} \mathrm{~s}$ and 2.2 and $4.2 \times 10^{12} / \mathrm{m}^{2} \mathrm{~s}$ for the inbound and outbound legs.

Some cometary material is undoubtedly plcked-up tallward of the ICE fly through distance as discussed above. This effect should account for an Increase in the actual loss rate of the comet as compared to the numbers calculated here. The numbers derived with this method, therefore, represent lower bounds on the total cometary loss rate. The amount of materlal picked-up tallward of the ICE traversal should be less than or equal to that plcked-up sunward and, therefore, we find a total cometary particle loss rate of 2.5-5 $\times 10^{28}$ molesules/s, in excellent agreement with the vartous Independently derived values quoted above. 


\subsection{Summary and Discussion}

In this study we have used the combined plasma and magnetic fleld data sets from the ICE traversal of Comet Glacobln1-Zinner to make a detalled examination of the configuration and properties of the draped G-Z magnetotall in general, and its fleld reversing current sheet in particular. The general interaction of the solar wind and embedded IMF with cometary bodies is well understood in terms of mass loading and, consequently, slowing of the flow, and draping of the magnetic fleld due to the velocity shear between reglons at varylng transverse distances from the obstacle. The detalls of the global interaction and magnetotall formation process, however, are at present not well understood and are of considerable Interest to the space physics community.

At the time of the ICE crossing the G-Z magnetotall was rotated $\sim 10.5^{\circ}$ In the normal aberration sense, and $\sim 9.9^{\circ}$ above the ecliptic plane. The large difference in Inbound and outbound tall lobe sizes can then be explained as a purely geometric effect due to the fact that ICE traversed the tall slightly off-center. The angle of the cross tall current sheet from the ecliptic plane $\left(\sim 43^{\circ}\right)$ derived purely from magnetic fleld measurements is in excellent agreement with that derlved with spacecraft timing under a clrcular tall cross-section assumption $\left(\sim 47^{\circ}\right)$, and this agreement suggests that the tall may be quite c1rcular. A c1rcular geometry is not expected from the near-nucleus lon pick-up process, and suggests that some circularization of the tall has occurred downstream from there. The 
residual ram pressure within the cometary environs could supply just such a circularizing effect.

The cross tall ( $\left.Y^{*}\right)$ component of the MHD momentum equation was used in the MHD Equilibrium section to df ive the unmeasured ion properties required to supply pressure balance across the tall. The calculated ion pressure is much greater than the electron contribution across the entire tall. The plasma beta vartes across the tall from $\sim 1-4$ in the lobes to $\sim 40$ in the central current sheet. The beta calculations can be used to denote the location of the Inbound and outbound edges of the tall where beta rises sharply.

Combining the electron plasma and magnetic fleld measurements with the X-components of the MHD equations made it possible to infer the plasma properties of the near-nucleus region where the average Ion is picked-up. These inferred properties, displayed in Figure 4.8, Indicate that the strongest mass loading is confined to a region only $\sim 1500 \mathrm{~km}$ across. In this narrow reglon the plasma flow velocity drops to $\sim-12 \mathrm{~km} / \mathrm{sec}$, a factor of $6-7$ slower than the flow about $1000 \mathrm{~km}$ farther on elther side of the obstacle. At the same time, the plasma density $18 \sim 200-600 / \mathrm{cm}^{3}$ in this region, a factor of 10-30 times that of the adjacent reglon. Similarly, the Inferred Ion source function, displayed in the bottom panel of Figure 4.8 , shows sharp rises at the edges of this region even though the function has been 1ntegrated over $7800 \mathrm{~km}$ from the average plck-up location back to ICE. If the primary mass loading region has an $\mathrm{x}$ extent of $1500 \mathrm{~km}$, similar to its $\mathrm{Y}^{-}$extent, then the approximate ratio of Ion source rates is $(7800 / 1500)$ times the ratio of values 
displayed in this panel $(0.7 / 0.15)$. Th1s calculation yields a ratio of Ion source rates inside/outside of the prime mass loading region of $\sim 24$.

Such a sharp gradient in the Ion source rate and related plasma parameters is perhaps somewhat unexpected. MHD cometary simulations such as those by Schm1dt and Wegmann [1980, 1982] and Fedder et al. [1986] assume that the source term is a spherically symmetric function of distance, $R$, from the comet's nucleus and 18 proportional to $R^{-2} \exp \left(-R / R_{0}\right)$. Therefore, such simulations should do a much poorer fob of modeling cometary magnetotalls than the more global cometary Interaction. Other models (e.g., Huebner [1985] and Bolce et al. [1986]) allow for detalled chemistry interactions in the cometary coma, and of ten show very strong gradients in total ion density (see for example Figure 3 in Huebner [1985]), but do not model the full MHD interaction with the inflowing solar wind. In the classical cometary plcture, and most of both types of models an impenetrable boundary or contact surface serves to separate the solar wind from the majority oi the lonized cometary material. Mendis et a1. [1986] calculate a boundary or lonopause radius of $\sim 600 \mathrm{~km}$ for Comet G1acobini-zinner at the time of the ICE crossing, which is very similar to our inferred radius of $\sim 750 \mathrm{~km}$ for the prime mass loading region.

In the classic picture of cometary magnetotall formation the magnetotail plasma sheet is simply a tailward extension of the near-nucleus lonospheric region, and is comprised of cold $\left(T_{1} \lesssim 1000^{\circ} \mathrm{K}\right)$ and totally unmagnetized lons. This is expected to 
occur because the near-nucleus lonization rate is substantially larger than the recombination or loss rate and, therefore, tallward Ion flow is required to maintain a steady state configuration. In this study we find no evidence for such an extended region of purely cold, Ionospheric plasma back at the ICE traversal distance of $\mathbf{- 7 8 0 0}$ $\mathrm{km}$. The magnetotall plasma sheet or current sheet data from G-Z Indicate that the region $1 \mathrm{~s}$ magnet1zed throughout, and is comprised of lons about 100 times hotter $\left(\sim 10^{5} \mathrm{~K}\right)$ than typical expected lonospheric temperatures. For the tall to maintaln 1 ts form $\sim 8000$ $\mathrm{km}$ downstream from the comet nucleus at least a rough pressure balance must, exist and, therefore, relatively hot ion temperatures within the current sheet are unavoldable since the electron pressure 1s so small.

While no evidence exists for an extended region of purely cold lonospheric plasma at the ICE downstream traversal distance a well formed lonospheric obstacle upstream, near the nucleus, may be present. In1tial results from the Comet Halley encounters at varying distances upstream from the nucleus (see the special Nature 1ssue [Vol. 321]) demonstrate the existence of the; cold Ionospheric region which is, at most, only slightly magnetized $(|\mathrm{B}| \lesssim 2 \mathrm{nT})$. Furthermore, the simflarity of the calculated fonopause radius and the inferred radius of the prime mass loading region suggests that the two may be closely related.

One possible explanation for both the observed current sheet properties and the comparatively large source rate inferred in the prime mass loading region upstream is based on the penetration of 
the solar wind through the cometary lonopause and Into the cometary 1onosphere. While most models assume the cometary lonopause to be perfectly impenetrable, this seems rather unlikely. Stagnation streamlines may have sufficiently slow flow that the magnetic fleld has time to diffuse Inward as $1 \mathrm{~s}$ observed at Venus, although the convective flow is outward from the nucleus rather than inward toward the planet. Alternately, other processes such as the Kelvin-Helmholtz instability may act at the cometary ionopause allowing penetration of the fleld and flow as suggested by Ershkovich and Mendis [1983]. The solar wind flow and fleld which partlally penetrates the lonopause would have access to the much larger lonospheric ton densitles, and consequently larger source rates. The ICE observation that the magnetic field has a substantial component across the tall and the large lon temperature Inferred from a pressura baliance condition indicate that at least $7800 \mathrm{~km}$ back in the tall, no extension of an lonopause boundery which delineates a region of cometary lons that have never been plcked-up by the solar wind was encountered. Rather, unless this region is very narrow, and was simply missed by ICE, the observations Indicate that current sheet lons, which pass the ICE traversal distance, may all have been plcked-up within the higher density region at the cometary lonosphere.

Another possiblifty is that the large source rate inferred in the prime mass loading region could be caused by physical variations In the neutral source structure at the nucleus. Observations by the Vega and Glotto spacecraft show clear evidence for gas/dust jets 
emanating from the sunlit side of the nucleus, and fissuring of the nucleus surface. Such jets could have the effect of forming very strong gradients in the neutral, and therefore Ion, densities. Such an enhanced region could account for the large source rate in the prime mass loading region as demonstrated by our analysis.

Finally, the coma chemistry models could account for the large Ion source rate. Complicated cometary chemistry processes extend out to some thousands of $\mathrm{km}$ from the cometary nucleus, and provide strong gradients in certain lonic abundances. It could simply be that the prime mass loading region is demarked by particularly strong gradients in the ion density.

For whatever reason the plasma flow is preferentially mass loaded within a small region upstream the implications of this region for the formation, density, and length of the cometary magnetota11, and visible Ion tall (magnetotall current sheet) are fundamental. At the distance of the ICE traversal the tallward plasma velocity is relatively constant at $\sim-20 \mathrm{~km} / \mathrm{sec}$ across the entire ta11. Upstream, at the average plck-up location along each streamline, the flow in the prime mass loading region is much slower than that upstream from the lobes and immediately adjacent portions of the lonlzed coma. Therefore, the velocity shear, which causes the draped magnetic configuration, and thereby the magnetotall 1tself, must occur upstream from the ICE encounter where current sheet velocities are appreclably smaller and lobe and lonized coma velocities appreclably larger.

The ICE downtall traversal distance curns out to be a 
fortuitous area for the spacecraft to have passed through for magnetotall studies, In that the flow velocity is relatively constant across the tall. The plane that ICE crossed the tail in, therefore, essentially separates the reglons of tall formation and dissipation. Beyond this distance the still accelerating plasma in the current sheet should be overtaking the surrounding plasma and the field configuration should be reapproaching a straight (nondraped) configuration.

The 1uferred approximate flow speeds in the current sheet (near the edges of the lobes) in the prime mass loading region and at ICE

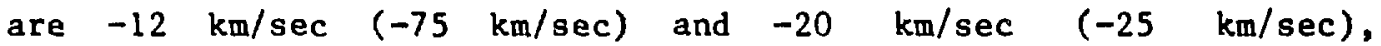
respectively. The average flow velocttles between these two locations are, therefore, $\sim-16 \mathrm{~km} / \mathrm{sec}$ and $\sim-50 \mathrm{~km} / \mathrm{sec}$. Using these average speeds, the times for an average plck-up ion to reach the ICE crossing plane are $\sim 8.1$ and $\sim 2.5$ minutes for the current sheet and lobes, respectively. This indicates that the plasma in the current sheet is lagging the plasma in the lobes by $\sim 5.6$ minutes.

If the current sheet plasma continues to accelerate at $\sim 8$ $\mathrm{km} / \mathrm{sec}$ in $\sim 8$ minutes, and the lonized coma velocity beyond the major mass loading region stays fixed at $\sim-25 \mathrm{~km} / \mathrm{sec}$, the draped magnetotall configuration would be entirely dissipated (1.e., the field would be completely stralghtened out) within some tens of thousands of kllometers downstream from the nucleus. of course, as the field restraightens the $\overline{\mathrm{J}} \times \tilde{B}$ force acting to accelerate the plasma is reduced so that a slightly draped configuration should be expected to extend out to somewhat greater distances. 
As the current sheet plasma accelerates, 1ts density decreases in order to conserve the number flux. In addition, as the fleld configuration straightens, the current sheet plasma becomes less confined in the $\mathrm{Y}^{-}$-direction, and plasma pressure gradients and particle parallel velocities tend to spread out the plasma in the current sheet and further decrease 1ts density. As the current sheet density decreases with disfance, the tall becomes less visible. The tall scale length given above is in basic agreement with the fact that a visible Ion tall was observed in CCD Images from earth in the $\mathrm{H}_{2} \mathrm{O}^{+} 619.8 \mathrm{~nm}$ line some $9600 \mathrm{~km}$ tallward from the nusleus at the time of the ICE encounter [Strauss et al., 1986], as discussed in greater detall by Slavin et al. [1986b] and MeyerVernet et al. [1986c]. Our calculated tall scale length is also in agreement with the general lack of observations of a tallward lonic extension $\mathrm{frcm} \mathrm{G-Z}$ greater than a few tens of thousands of $\mathrm{km}$.

At more active comets such as Halley, which has a neutral gas production rate $\sim 20-40$ times that of $G-2$, the solar wind must become even more slowed in the prime mass loading region, and the fleld configuration must become more highly kinked. Without knowing the decalls of how an enhanced gas production rate would affect the prime mass loading region versus the immediately surrounding region, it is difficclt to ascertain exactly how the comet's tall would be affected. However, it is clear that such a comet should have substantially slower current sheet flows. Since the density is proportional to the gas production rate, and inversely proportional to the flow velocity, ion densitles in such fon talls should be 
substantially enhanced over $G-Z^{\circ} s$, and these talls should be much more visible. The length of more active comets talls should also be appreclably greater slnce any further slowing near the nucleus would cause the current sheet plasma to fall even further behind the lobe flow throughout the near portions of the magnetota11. For any reasonable $\tilde{J} \times \bar{B}$ reacceleration rate this difference should take appreclably longer to be made up.

Smaller comets such as G-Z may also go through active phases Jue to surface effects such as cracking and large variations in the gas production rate. Evidence described above for observations of surface fissures and large scale gas/dust jets at comet Halley Indicate that the cometary source function can be far from constant or symmetric. In addition, variations in the upstream solar wind parameters undoubtedly have an effect on tall formation, and one might expect that higher speed flows would enhance the tail formation and field draping process by increasing the plasma flow outside of the prime mass loading region. Such variations might account for the difference between this apparition of $G-Z$ and the last, in 1959, which regularly exhibited a long visible Ion ta11.

While the very small prime mass loading region has an ion source rate $\sum_{24}$ times that anywhere else in the extended cometary environs, and is apparently responsible for producing the narrow, well-defined comet ta11, the total, Integrated mass addition in this small area is $\leqslant 1 \%$ of the steady state mass added to the flow throughout the extended cometary region. Consequently, the most visible aspect of cometary apparitions, the lon tail, or magnetotall 
current sheet, is relatively unimportant in the global cometary process of mass loading the solar wind, and removing the large cometary efflux.

The generally good agreement between global cometary models and the results from the ICE Glacobla1-21nner encounter 1ndicate that the detalled physics of the comet nucleus and near-nucleus region are not of fundamental Importance to the large scale cometary Interaction and the majority of the cometary mass plck-up. This study, however, indlcates that a detalled knowledge of the nucleus and near-nucleus region are of fundamental importance to the understanding of the magnetotall formation process and the large variations in visible ion talls which have intrigued mankind for millenia. We look forward to the many valuable and detalled studies which will undoubtedly spring from the Halley armada data sets upstream from the comet nucleus, and hope that a truly fundamental knowledge of comets and cometary nuclel w111 be made attainable soon through the reallzation of the Comet Rendezvous Asterold F1yby (CRAF) mission.

\subsection{Chapter Acknowledgments}

We gratefully acknowledge comments and suggestions on this paper from C. T. Russe11, J. G. Luhmann, M. F. Thomsen, and R. D. Zwlckl and are particularly grateful to G. L. Siscoe for his many valuable suggestions. The research performed at the Jet Propulsion Laboratory of the California Institute of Technology was carried out under contract to NASA, while work done at Los Alamos 
was performed under the ausplces of the United States Department of Energy with support from NASA under S-04039-D. 


\section{Chapter 5}

Summary and Discusston

\subsection{Summary}

Planetary and cometary magnetotalls are some of the largest, most interesting, and most often observed manifestations of the solar wind's interaction with solar system bodies. Generally, magnetotalls are formed out of the flowing solar wind's interaction with various relatively stationary MHD obstacles.

Although there are a number of general simflarities between the mantfestations of magnetotalis and their fleld rever: Ing current sheets at the earth, Venus, and Comet Glacobinf-Zinner, many important differences also exist. These are based on fundamental differences in the nature of the planetary/cometary bodles and, therefore, the obstacles they present to the solar wind. The numerous new results of this dissertation, highlighted in the three sections below, represent a wealth of new information about the detailed manifestations of the magnetotalls and fleld reversing magnetotall current sheets at these three planetary/cometary bodles. Through the detalled examination of the various magnetotall and current sheet configurations and properties, it is hoped that this dissertation has served to convey some of the richness of varlety which magnetotalls and their field reversing current sheets display throughout the solar system. 


\subsubsection{Earth}

The section on the terrestrial magnetotall (Chapter 2) examines the detalled geometries and current density distributions in the near earth cross tall current sheet during an interval of relative geomagnetic quiet. In this way a typical, time independent configuration of the tall is studied, and the details of how the currents flow within the current sheet are determined. The major new accomplishments and results of this section are summarized here.

1) Combined magnetic fleld and three-dimensional plasma data from the co-orbiting satellites ISEE-1 and -2 were used to examine the current sheet geometries at the times of three sheet crossings. From the measured fleld orlentations, the Inclinations of the field lines within the current sheet were Inferred. This analysis ylelded inclinations of $15^{\circ}, 59^{\circ}$, and $72^{\circ}$ and indicasizs that large field inclinations within the current sheet may be cownonplace, even at relatively geomagnetically inactive times. Further, this work stimulated an equilibrium analysis of the magnetotall [Volgt and Hilmer, 1986] which theoretically explains the large observed Inclinations.

2) A new data analysis technique was developed in this section tu calculate the variable current sheet crossing velocities from the co-orbiting, dual satellite, magnetometer data sets, and the inferred field inclinations. This technique allows the spatial varlations in the fleld to be separated from temporal fluctuation, and thereby resolves them. 
3) Integrating the varlable velocity profiles over the 1ntervalis of the current sheet crossings ylelds current sheet thicknesses wh1ch are determined Independent of sheet motions for the first t1me. For the relatively quiet cases studied here the total sheet th1cknesses are in the range from $\sim 1-5 \times 10^{4} \mathrm{~km}$ (tens to hundreds of ion gyroradii), where the thinner sheets are assoctated with cases of larger fleld Inclinations.

4) While the current sheet is thick on the size scale of an Ion gyroradius, this chapter demonstrated that it is appreciably thinner than the plasma sheet as defined by the plasma data. The cross tail current sheet and piasma sheet are, therefore, separate entities, with the current sheet being embedded in the thicker plasma sheet.

5) Separation of the spatial and temporal variations of the magnetic field through the current sheet crossings made it possible to calculate the detalled current density distributions in the cross tall current sheet for the first time. These distributions are displayed in Figures 2.6, 2.9, and 2.10, and the peak current densities range from $\sim 5-50 \mathrm{nA} / \mathrm{m}^{2}$.

6) Current density distributions calculated in this section appear to be characterized by two distinct regions. The central $\sim 1 / 3$ of the distributicns contain relativeiy large current densities which peak very near to the center of the current sheet. Outside this region the distributions generally display shoulders of lesser current densities which decay with outward distance. 
7) Superimposed on the general shapes of the distributions there is considerable fine scale structure which produces large amplitude variations in the current density with scale sizes $\sim 2-6 \times 10^{3} \mathrm{~km}$ (a few to tens of fon gyroradi1). These peaks may indicate that the cross tall current sheet consists of numerous fine scale current filaments, possibly caused by ion kinetic effects.

\section{0 .2 Venus}

The Venus section (Chapter 3) concentrates on determining the average configuration and properties of the Venus magnetotail. This goal had not been previously achieved due to the extremely large varlabllity of the tall location and magnetlc conflguration. In this dissertation the varlability is used to provide the statistical sampling needed to reconstruct the average, global, tall and current sheet configurations. The mafor new accomplishments and results of this section are summarlzed here.

1) Ploneer Venus Orbiter observations within the Venus magnetotall routinely show a great deal of varlabllity and, for example, the magnetic field data indicate that the spacecrait traverse? between oppositely directed lobes many times in a single orbital pass. Some of this varlabllity in apparent location within the magnetotail can undoubtedly be attributed to vartations in the upstream solar wind flow direction. A large portion of the varlability, however, must be attributed to another source. In this chapter the effects of draping a highly varlable upstream magnetic configuration into the tall were proposed to account 
for the varlability. As the upstream cone angle of the IMF fluctuates, the relative amounts of magnetic flux within the two tail lobes at any given downtall distance are highly varlable (see Figure 3.1). Therefore, the current sheet is forced to Elap from side to side within the Venus magnetotall in an effort to malntain a pressure balance condition across the tall. This large scale flapping occurs on the rapid timescale of upstream IMF fluctuations and has confused the results of previous studies of the Venus magnetotall, which order the observations by their locations in spacecraft coordinates.

2) The 9423 one-minute averaged magnetic field measurements used in this statistical study were examined for evidence of reverse draping within the Venus magnetotall. Seventy-nine percent of the observed data were shown to be in the sense of a normal, draped magnetotail while twenty-one percent pointed in a reversed draped sense. The same procedure was repeated on 2701 one-minute data samples of purely magnetosheath field, and reverse draping was observed in sixteen percent of those data indicating that that fraction of apparent reverse draping was probably due to simple field rotations and normal fieid draping. It was therefore concluded that only a very small fraction of the data was Indicative of actual reverse draping of the field ( $\leq 5 \%)$ and that magnetic ieconnection $1 \mathrm{~s}$ probably not an important physical process in the Venus magnetotail.

3) A good statistical correlation was found between the magnetic field draping angle and the relative magnitude of the field, 
compared to the field strength in the immediately adjacent portions of the magnetosheath. The cross tall current sheet was Identifled with the region of low relative fleld strength and with fleld vectors which polnt nearly across the tail (perpendicular to $X$ ) while the lobes were Identifled with reglons of large relative fleld strength and fleld vectors which point nearly parallel and antiparallel to the tall (X) axis.

4) The Venus magnetotall configuration was statistically confirmed to consist of only two draped magnetic lobes separated by a single, fleld reversing current sheet. The average field draping angles in the outer reglons of the lotes were determined to be $-78.4^{\circ}$ and $73.4^{\circ}$ measured from the cross-flow direction toward the $+\mathrm{X}$-direction. The $5^{\circ}$ difference between these two values is attributable to the effect of the average solar wind spiral angle draplng into the tall.

5) Based on the large varlability of the magnetic fleld data, a new statistical data analysis technique was developed. This technique uses uniform sampling sipplied by the large fluctuations in the location of the cross tall current sheet to determine the relative frequency of various fleld draping angles within the ta11. From these relative frequencles, and knowing the full width of the tail, a coordinate system was constructed which statistically determines the cross tall locacion ( $\left.Y^{*}\right)$ of each data point. In essence, these derived coordinates measure distances from the center of the cross tall current sheet rather than In spacecraft coordinates and thereby allow for a good 
determination of the average magnetic topology of the Venus magnetotall for the first time.

6) In the new, derived coordinates a number of magnetic field properties as functions of cross tall location were determined for the first time. In particular, the tallward/sunward magnetic fleld, $B_{X}$, and the cross tall magnetic fleld, $B_{Y *}$, were both determined as functions of $Y^{*}$. ${ }^{B} X$ was shown to vary smoothly from one lobe orlentation to the other through the current sheet wh1le $\mathrm{B}_{\mathrm{Y}}$ was found to be a double humped function, similar in appearance to cometary simulation results.

7) The extensive data set used in this study was also ordered according to downtall distance in order to find the average variation of $B_{Y *}$ with $X$. The best linear fit to this variation was derived to be $\mathrm{B}_{\mathrm{Y}^{*}}(\mathrm{X})$, averaged over $\mathrm{Y}^{*},=11.53+0.68 \mathrm{X}$, where the fleld is measured in $n T$ and $X$ is in $R_{V}$.

8) From the derived variations of $B_{Y *}$ as a function of $X$, and $B_{X}$ as a function of $Y^{*}$, both components of the cross tall current density, $J_{Z}$, as a function $Y^{*}$ are calculated. $J_{Z}$ was found to vary from $\sim 1.5 \mathrm{nA} / \mathrm{m}^{2}$ in the center of the current sheet to $\sim 0.1 \mathrm{nA} / \mathrm{m}^{2}$ at the edges of the current sheet.

9) Combining $B_{X}, B_{Y^{*}}$, and $J_{Z}$, all as functions of $Y^{*}$, gave the distribution of JxB forces across the tall. This electromagnetic force points tallward and Inward toward the tall center at all locations across the tall as displayed in F1gure 3.15.

10) From the derived, average magnetic field varlations down the 
tall, and the condition of continulty of the tangential electric fleld, the variation of tine bulk downtall plasma velocity and plasma acceleration were then determined. The plasma velocity and acceleration vary from $\sim-250 \mathrm{~km} / \mathrm{sec}$ and $\sim-1 \mathrm{~km} / \mathrm{sec}^{2}$ at $-8 R_{V}$ to $\sim-470 \mathrm{~km} / \mathrm{sec}$ and $\sim-7 \mathrm{~km} / \mathrm{sec}^{2}$ at $-12 \mathrm{R}$, respectively.

11) This derived plasma acceleration profile, JxB force distribution, and the MHD momentum equation were then combined to determine the average current sheet and lobe plasma densities $\left(\sim 0.9 \mathrm{amu} / \mathrm{cm}^{3}\right.$ and $\left.\sim 0.07 \mathrm{amu} / \mathrm{cm}^{3}\right)$ and the approxtmate average Ion temperature for the tall $\left(\sim 9 \times 10^{7} \mathrm{~K}\right.$ for a purely $0^{+}$tall). The typlcal plasma parameters derived in this chapter represent the only avallable data on the average plasma properties of the Venus magnetotall since none of the PVO plasma analyzers has a sufficlent sensitivity and energy range to resolve the plasma properties. The average properties derived here ware shown to be unobservable by the PVO plasma Instrumentation, and therefore to be consistent with their general lack of observations.

12) The plasma betas calculated from the derived plasma properties are $\sim 10$ for the current sheet and $\sim 0.1$ for the lobes. These results are consistent with the expectation that varying quantities of magnetic flux at a given downtall distance will cause the cross tall current sheet to move within the tall and account for the large observed varlability of the magnetic fleld data.

13) From the derived tallward plasma velocity and lobe and current sheet plasma densities, the mass flux down the Venus magnetota11 
was calculated. This flux, integrated over the whole ta11, is $\sim 1 \times 10^{26} \mathrm{amu} / \mathrm{sec}$, and sets a reasonable bound on the neutral gas loss rate of the Venus atmosphere.

\subsubsection{Comet Giacob1n1-Z1nner}

The Comet Giacobini-zinner section (Chapter 4) combines the high resolution magnetic fleld and plasma electron data from the ICE tail traversal, for the first time. With the combined data and using the MHD equations, the detalls of the G-Z magnetotall are examined, and 1ts configuration and properties are derived. The major new accomplishments and results of this chapter are summarized here.

1) Plasma electron moments from the Immedlately adjacent regions of solar wind, Inbound and outbound along the ICE trajectory, were Interpolated across the cometary encounter 1nterval. This procedure ylelded best upstream values for the plasma moments at the time of the ICE tall encounter $(\sim 11: 00)$ of $n_{\mathrm{SW}}=6 / \mathrm{cm}^{3}$, $v_{S W}=460 \mathrm{~km} / \mathrm{sec}$, and $\sim-10.5^{\circ}$ solar wind flow angle (in the sense of normal aberration). These values set the upstream conditions for the tall crossing interval.

2) Using the interpolated flow angle and the Inbound and outbound tail boundary crossings, the orientation of the magnetotall at the time of the ICE traversal was determined under a circular ta11 cross-section assumption. The ortentation of the current sheet within the tail was then calculated using the additional assumption that the current sheet bisects the tail, and the 
result was an angle of rotation of the current sheet within only $4^{\circ}$ of the $43^{\circ}$ angle derlved from the fleld data. This agreement indicetes that the two assumptions made about the tall configuration were quite good. The large difference between the Inbound and outbound lobe crossing Intervals was then simply explalned in our derived tall/ICE crossing geometry by the slightly off-center trajectory of the spacecraft through the tall.

3) The total pressure on the external surface of the tall was derived from Bernoulit's equation and the upstream plasma conditions. This derived pressure is $\sim 1.2 \times 10^{9} \mathrm{~N} / \mathrm{m}^{2}$ and supplied the boundary condition on the pressure balance within the tail.

4) The pressure balance condition within the tall was derived from the cross tail component of the MHD momentum equation and applied to the magnetic field and plasma electron measurements across the tall. Th1s analysis gave the required Ion pressure (and therefore temperature) profile for a balanced, steady state condition to exist across the tall. The derived ion pressure is much greater than the electron pressure and varies in an Inverse sense from the magnetic field pressure.

5) The tallward/sunward component of the electromagnetic JxB force was caiculated across the tail from smoothed magnetic fleld data, and the distribution was shown to consist of two distinct reglons. In the current sheet the force is tallward, Eaccelerating the most heavily mass loaded portions of the 
fleld, while in the outer lobes the force is sunward, Indicating that the lobe fleld is still being stretched and laminated into the draped tall configuration.

6) The number flux of particles passing the ICE location along any streamline was shown to be twice that of upstream at the average pick-up location on that streamline. This relation has 1mportant Implications for the properties upstream, near to the comet nucleus. Beyond the average pick-up location along each streamline mass addition continues to slow the plasma flow.

7) The measured fleld and electron properties and derived ion properties and JxB force across the tall were combined with the axial component of the MHD momentum equation, the relation between the upstream and local number fluxes, the relation between pick-ıp velocity and temperature, and the MHD energy equation. This analysis ylelded four equations in four unknowns which were solved with an iterative computer technique. Outputs from this analysis provided the first determinations of 1) the flow velocity profile at ICE, 2) the velocity profile upstream at the average pick-up location, 3) the Ion temperature profile upstream at the average pick-up location, and 4) the density profile upstream at the average pick-up location.

8) The flow velocity at ICE was shown to be quite constant ( -20 to $-30 \mathrm{~km} / \mathrm{sec}$ ) across the entire tall. This indicates that the ICE traversal distance was a spectal region for the spacecraft to have flown through, in that tall formation is occurring sunward 
of this distance while tall dissipation must be occurring tallward from $1 t$.

9) The plasma properties in the average upstream region where tons are plcked-up along each streamline are very different in regions upstream from the current sheet versus upstream from the lobes. Upstream from the current sheet our analysis finds a region of prime mass loading where the lon density rises to $\sim 400$ to $500 / \mathrm{cm}^{3}$ while the plasma flow speed drops to only $\sim-12 \mathrm{~km} / \mathrm{sec}$. Upstream from the lobes, at the average plck-up locations, the density is $10 \mathrm{w}\left(\sim 20 / \mathrm{cm}^{3}\right)$ and the $\mathrm{flow}$ speed high $(\sim-75 \mathrm{~km} / \mathrm{sec})$.

10) The difference between the prime mass loading region upstream from the current sheet and the regions upstream from the lobes is further accentuated by the calculation of the source term in the MHD continulty equation from the divergence of the mass flux with downtall distance. This calculation yielded a region where the ion source rate is $\sim 24$ times that of the regions only $\sim 1000 \mathrm{~km}$ to either side of $1 \mathrm{t}$. Some possible causes of such a regton were discussed and, in particular, the possibility of it being due to diffusion into the cometary lonosphere was examined. This model was also substantiated by the lack of an extended region of purely cold, unmagnetized lonospheric ions back at the ICE traversal distance.

11) The approximate acceieration rates of the lobe and current sheet plasmas between the average pick-up lozations and ICE were used to examine the approximate scale length of the G-Z magnetotall. 
The result of a few tens of thousands of k1lometers is in good agreement wlth earth-based optical observations. In addition, the Implications of the prime mass loading region and calculated acceleration rates were examined to attempt to describe why some comets have extensive visible ion talls and others do not.

12) The total number flux of particles traveling down the G-2 tall was calculated from the observed density and calculated velocity distributions. This integrated flux is $2.6 \times 10^{26} \mathrm{H}_{2} \mathrm{O}^{+} / \mathrm{sec}$, and represents only $u 1 \%$ of the total mass loss rate of the comet. Consequertly, the highly visible Ion tall is relatively Inconsequential in the global process of the solar wind's removal of material form the comet.

13) In order to find which locations are important in the more global mass plck-up and removal process, the number flux of particles for the entire ICE encounter was analyzed. The total derived Ion loss rate for the entire encourtter was found to be $\sim 2.5-5 \times 10^{28} \mathrm{H}_{2} \mathrm{O}^{+} / \mathrm{sec}$ in excellent agreement with values derived by other, Independent techniques. The dominant mass loading appears to occur in the large fluctuations of the transition region and outer magnetosheath.

\subsection{General Discussion}

Despite the very large differences between the three budies which form the magnetotalis studied in this dissertation, there are a number of common aspects which merit further discussion. This final section compares the general configurations, use of data, and 
a number of specific results from the three chapters with the intent of elucidating their commonality. Each of the three main chapters in this dissertation was written in such a way that it can stand alone as a separate Journal of Geophysical Research paper. Consequently, each of the three also contalns separate discussions of how the results fit into the broader plcture of space plasma physics.

All three magnetotalls examined in this dissertation consist of high beta current sheets which serve to separate two lower beta lobes of essentially oppositely directed magnetic fields. In all cases this general topology is traceable to the flow of the solar wind past the obstacle. The upstream solar wind (or magnetosheath flow if a shock is formed) can interact with the obstacle In a number of ways: magnetic interconnection between the IMF and planetary fleld (earth); viscous drag along the magnetopause (earth) or lonopause (Venus and comets) boundarles; mass loading of the flow (Venus and comecs). In all of these cases, however, momentum and energy are transferred from the solar wind flow to the bodies with the effect of stretching out magnetic flelds roughly parallel and anti-parallel to the solar wind flow vectrr.

This roughly antiparallel field configuration requires the formation of a self-consistent field reversing magnetotall current sheet where cross tall currents flow in accordance with Ampere's Law. Within the current sheets, plasma densitles and betas are relatively large, and the fleld undergoes a rotation from one lobe's orientation to the other. Electromagnet1c $3 \times B$ forces in 
magnetota1ls in general, and within their current sheets in particular, act to reduce the kinking of thi: fleld configuration efther to a dipole (near earth) or stralght IMF (distant earth, Venus, and comets) configuation, and thereby disst.pate the tall. only the continued Input of energy and momentum from the solar wind flow can act to sustain the tall configurations as the long lived MHD structures whtch populate the solar system.

The earth, Venus, and G- Z data sets tach provided quite dfferent opportunities for examining the structure and properties of the three magnetotalls and their fleld reversing current sheets. At Venus the data set contained $\sim 10^{4}$ one minute averaged magnetic field data samples, and allowed for the reconstruction of the average magnetic configuration of the Venus magnetota1l. At earth and Comet $G-Z$, case studies and a single encounter traversal were used to examine the detalled tail structures on a crossing by crossing basis. In the earth study, magnetic fleld data from two co-orbiting satellites were combined to separace the spatial and temporal varlations of the plasma sheet, while in the G-Z study, two plasma electron data sets were combined with the fleld data to derlve the properties of the average plck-up location upstream. In all three studies, multiple measurements, either statistically with a single instrument or Individually with multiple 1astruments, were employed to determine the magnetotall structures in far more detail than would be possible with any of the single data sets alone. 
the Importance of these varlous dynamic effects, and determine the approximate time independent configuration of the ta11s.

The magnetic fleld orlentations in all three magnetotalls were found to be strongly affected by the angle of the upstream IMF in the plane perpendicular to the solar wind (clock angle). Fleld Iines in the terrestrial current sheet crossings studied were Inclined with respect to the nominal current sheet orlentations, and were found to generally point in the sense of the upstream IMF. Evidently the partial magnetic interconnection between the earth's field and the IMF allows the orlentation of the upstream configuration to affec't the internal magnetic field orientation (probably by setting up convection cells in the lobes). At Venus and Comet Glacobini-Zinner, the situation is even much more clear cut. Draping of the upstream fleld forms the field reversing current sheets, and, consequently, current sheet axes at these bodies are necessarlly orlented normal to the IMF directions.

Current sheets in the three magnetotalls studied here were all found to be thick compared to the asymptotic thermal ion gyroradif for those talls. The typlcal thicknesses range from $\sim 50^{+}$gyroradil at Venus to $\sim 19 \mathrm{H}_{2} \mathrm{O}^{+}$gyrorad11 at $\mathrm{G}-\mathrm{Z}$ and $\sim 30-100$ proton gyrorad11 for the central terrestrial current sheet. All of these values are larger than might be expected from the simple minded plcture of lons gyrating back and forth across a Harris type neutral sheet. Other physical processes such as gradient drifts of the particles, and the effects of a normal component of the fleld are undoubtedly also Important in these current sheets. 
Normal field components are observed at all three current sheets. These components range from $\sim 4 \mathrm{nT}$ at Venus to $\sim 10 \mathrm{nT}$ and G-2 and $\sim 10-20 \mathrm{nT}$ for the current sheet crossings studied at the earth. Compared to the typlcal lobe field values in the talls, these normal components are $\sim 1 / 3, \sim 1 / 4$, and $\sim 1 / 2$, respectively. Such strong normal components are Indicative of tall configurations which are relatively stable locally against reconnection.

In the case of G-Z, very few of the high resolution fleld vectors ( $1 / 3$ second) observed along the ICE trajectory were pointed In the opposite sense to the average field direction, while at Venus, only $\sim 5 \%$ of the fleld vectors were pointed in such a manner. These results Indicate that at least at the time and location that ICE crossed the G-2 current sheet, and generally in the -8 to-12 $R_{V}$ range of the Venus tail, reconnection is not a dominant physical process. Beyond the ICE intercept distance at G-Z and the range of tall coverage at Venus, the two draped field configurations in these magnetotalls should be restralghtening, and conditions for reconnection should be becoming even poorer. In contrast, while the near earth reglons of the terrestrial tail are not conducive to reconnection at the geomagnetically quiet times studied here, a more tall-like configuration (with decreasing normal component) occurs with distance down the tall. At some distance, reconnection must occur, on average, in order to malntain a steady state interconnection between the earth's field and the IMF which is constantly belng convected past. This deep tall reconnection may have Important Implications for energization of current sheet 
particles at earth, while at induced magnetotails Ions are energized primarily by the plck-up process.

Peak cross tail current densities determined for the three current sheets vary over a range of about 100 . The typlcal earth, Venus, and $G-2$ peak cross tall current densities are $\sim 5-50 \mathrm{nA} / \mathrm{m}^{2}$, $\sim 1.5 \mathrm{nA} / \mathrm{m}^{2}$, and $\sim 100 \mathrm{nA} / \mathrm{m}^{2}$, respectively. At Venus and $\mathrm{G}-\mathrm{Z}$ these cross tall currents give rise to peak tallward JxB forces of $\sim 1 \times 10^{-17} \mathrm{~N} / \mathrm{m}^{3}$ and $\sim 1 \times 10^{-15} \mathrm{~N} / \mathrm{m}^{3}$. Not surprisingly, the derived mass flux, or mass loss rate down the $G-Z$ tall is $\sim 50$ times that at Venus $\left(\sim 5 \times 10^{27} \mathrm{amu} / \mathrm{sec}\right.$ versus $\left.\sim 1 \times 10^{26} \mathrm{amu} / \mathrm{sec}\right)$, indicating that a much more mass loaded plasma exists in the G-Z tall, consistent with the much larger JxB force. Even though the G-Z magnetotall conducts away only $\sim 1 \%$ of the total cometary efflux, the near nucleus mass loading which accounts for the tall is still far more effective than the mass loading of the gravitationally bound Venus atmosphere and Ionosphere. The near-nucleus mass loading environment at $\mathrm{G}-\mathrm{Z}$, while not gravitationally bound, exhibits a region of concentrated mass loading which in some ways mimics the Venus obstacle. This region evidently accounts for the narrow, well-defined nature of the tall, and greatly enhances the similaritles between the solar wind Interactions with Venus and Comet Glacobini-Zinner.

Field reversing current sheets comprise a fascinating class of space plasma physics structures within the solar system, and quite possibly in many astrophysical applications. In the magnetotalls of the earth, Venus, and Comet Glacobini-Zinner, these structures present numerous interesting properties which have been examined in 
detall in the work of this dissertation. Knowledge gained from these examinations paves the way to a lifetime of research to better understnad the vast and st111 11ttle known realms of current sheets and other plasma structures throughout the solar system and beyond. 
The dissertation of David John McComas is approved.
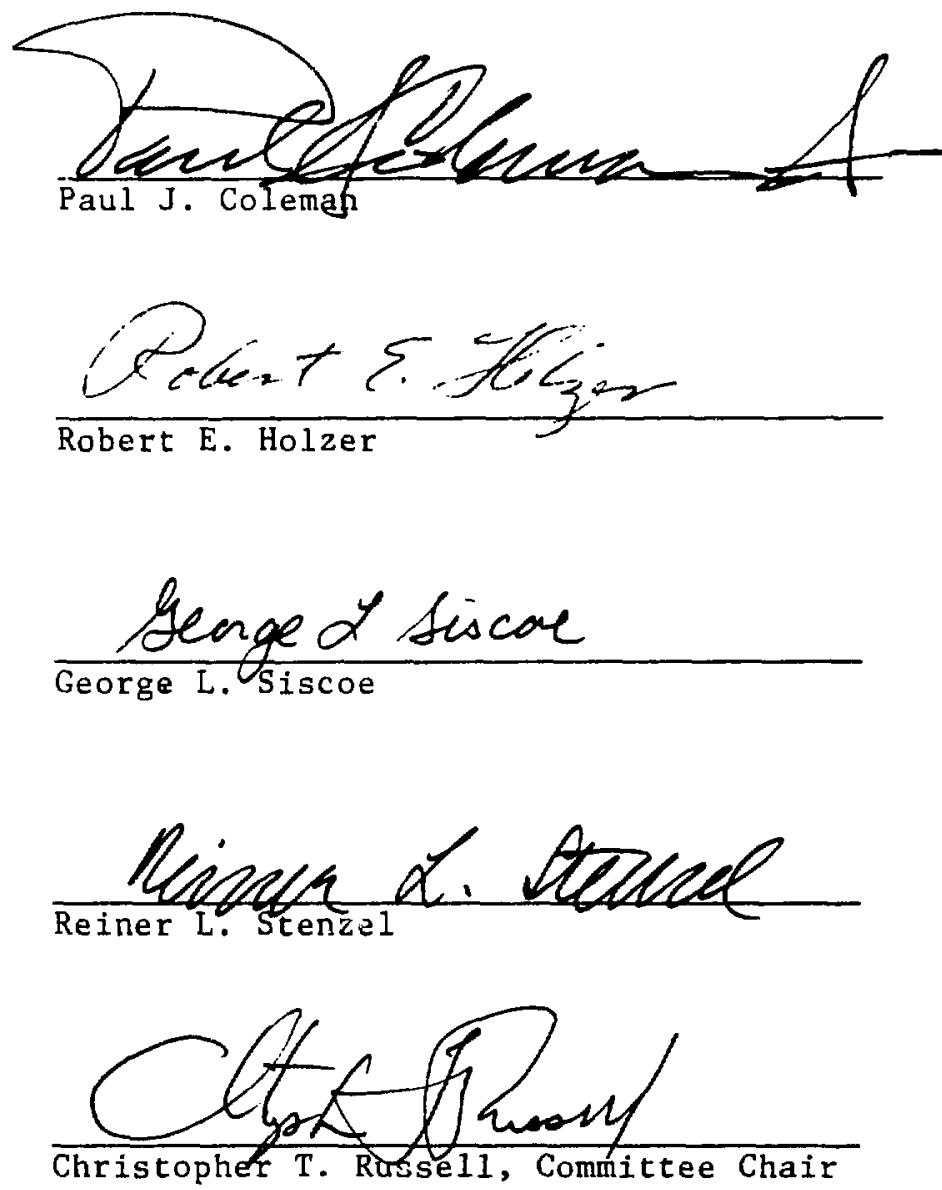

University of California, Los hngeles

1986 


\section{ACKNOWLEDGMENTS}

The American Geophysical Union is gratefully acknowledged for granting permission to reproduce material in Chapters 2 and 3 . ("The Near Earth Cross Tail Current Sheet: Detailed ISEE-1 and -2 Case Studies," McComas, D. J., C. T. Russell, R. C. Elphic, S. J. Bame; J. Geophys. Res., 91, 4287-4301, 1986, copyright by the American Geophysical Union; and "The Average Magnetic Fiela Draping and Consistent Plasma Properties of the Venus Magnetotail," McComas, D. J., H. E. Spence, C. T. Russe11, M. A. Saunders; J. Geophys. Res., 91, 7939-7953, 1986, copyright by the American Geophysical Union.) I an sincerely thankful to my co-authors on these papers, and to J. T. Gosling, S. J. Bame, J. A. Slavin, E. J. Smith, and J.-L. Steinberg, co-authors of "The Giacobini-Zinner Magnetotail: Tall Configuration and Current Sheet," submitted for publication. To C. T. Russell, W. I. Newman, and G. L. Siscoe, who provided invaluable graduate Instruction and guidance on all aspects of this dissertation, and to my wife, Richelle Wolff McComas, who provided tireless clerical and editorial support, I wish to give special thanks. Finally, I gratefully acknowledge Los Alamos National Laboratory, operated under the auspices of the United States Department of Energy, and my supervisors, D. N. Baker and W. D. Evans, and P.J. Coleman, head of the Los Alamos Branch of the Institute for Geophysics and Planetary Physics, for providing financial and administrative support throughout my graduate education. 
References

Alexander, C. J. and C. T. Russell, Solar cycle dependence of the location of the Venus bow shock, Geophys. Res. Lett., 12, 369, 1985 .

Alfven, H., On the theory of comet talls, Tellus, 9, 92, 1957.

Baker, D. N., E. W. Hones, Jr., J. B. Payne, and W. C. Feldman, A high-time resolution study of interplanetary parameter correlations with AE, Geophys. Res. Lett., 8, 179, 1981.

Bame, S. J., J. R. Asbridge, H. E. Felthauser, R. A. Olson, I. B. Strong, Electrons in the plasma sheet of the Earth's magnetic tall, Phys. Rev. Letters, 16, 138, 1966.

Bame, S. J., J. R. Asbridge, H. E. Felthauser, E. W. Hones, Jr., I. B. Strong, Characteristics of the plasma sheet in the Earth's magnetota11, J. Geophys. Res., 72, 113, 1967.

Bame, S. J., J. R. Asbridge, H. E. Felthauser, J. P. Glore, H. L. Hawk, and J. Chavez, ISEE-C solar wind plasma expertment, IEEE Trans. Geosc1. Electr., GE-16, 160, 1978.

Bame, S. J., R. C. Anderson, J. R. Asbridge, D. N. Baker, W. C. Feldman, J. T. Gosling, E. W. Hones, Jr., D. J. Mc.Comas, and $R$. D. Zwick1, Plasma reglmes in the deep geomagnetic tail: ISEE-3, Geophys. Res. Lett., 10, $912,1983$.

Bame, S. J., R. C. Anderson, J. R. Asbridge, D. N. Baker, W. C. Feldman, S. A. Fuselier, J. T. Gosling, D.J. McComas, M. F. Thomsen, D. T. Young, and R. D. Zwick1, Comet Glacobini-Zinner: A plasma description, Sclence, 232, 356, 1986.

Bargatze, L. F., D. N. Baker, R. L. McPherron, and E. W. Hones, Jr., Magnetosphertc Impulse response for many levels of geomagnet1c activity, J. Geophys. Res., 90, 6387, 1985.

Bleber, J. W. and E. C. Stone, Energetlc electron bursts $1 \mathrm{n}$ the magnetopause electron layer and in interplanetary space, in Magnetospheric Boundary Layers edited by B. Battrick pp. 131-135, ESA, Par1s, 1979 .

Blermann, L., Kometenschwelfe und solare korpuskularstrahlung, Z. Astrophys, 29, 274,1951 .

Bolce, D. C., W. F. Huebuer, and J. J. Keady, A model of Comet P/G1acobin1-Z1nner, Geophys. Res. Lett., 13, 381, 1986. 
Brandt, J. C., Observations and dynamics of plasma talls, in Comets, edited by L. L. Wilkening, University of Arizona Press, Tucson, 1982 .

Cloutler, P. A., R. E. Darleli, and D. M. Butler, Atmospheric Ion wakes of Venus and Mars in the solar wind, Planet. Space Sc1., 22, $967-990,1974$.

Cloutler, P. A., Solar wind interaction with planetary lonospheres, in Solar Wind Interactions with Planets Mercury, Venus and Mars,

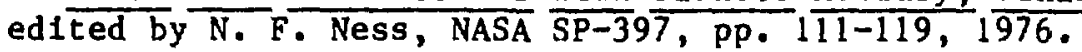

Cowley, S. W. H., The adlabatic flow model of a neutral sheet, Cosmic Electrodynamics, 2, 91,1971 .

Cowley, S. W. H., A self-consistent model of a slmple magnetlc neutral sheet system surrounded by a cold, collisionless plasma, Cosmic Electrodynamics, 3, 449, 1972 .

Daly, ".H., T. R. Sanderson, K. -P. Henzel, S. H. H. Cowley, R. $i$ Hynds, and E. J. Smith, Gyroradius effects on the energetic lons in the tall lobes of Comet P/Giacobini-zinner, Geophys. Res. Lett., 13, 419, 1986.

Dolginov, Sh. Sh., L. N. Zhuzgov, V. A. Sharova, and V. B. Buzin, Magneilc field and magnetosphere of the planet Venus, Cosmic Res., 16, 657, 1978 .

Ershkovich, A. I., and D. A. Mendis, On the penetration of the solar wind Into the cometary Ionosphere, The Astrophys. J., 269, 743, 1983.

Falrfield, D. H., On the average configuration of the geomagnetic ta11, J. Geophys. Res., 84, 1950, 1979.

Falrfield, D. H., A statistical determination of the shape and position of the geomagnetic neutral sheet, J. Geophys. Res., 85, 775,1980 .

Falrfield, D. H., R. P. Lepping, E. W. Hones, Jr., S. J. Bame, and J. R. Asbridge, Simultaneous measurements of magnetotall dynamics by IMP spacecraft, J. Geophys. Res., 86, 1396, 1981.

Fedder, J.A., J. G. Lyon, and J. L. Glulianl, Jr., Numerical simulations of comets: predictions for Comet Giacobini-Zinner, EOS, $67,17,1986$.

Forbes, T. G., E. W. Hones, Jr., S. J. Bame, J. R. Asbridge, G. Paschmann, N. Sckopke, and C. T. Russe11, Substorm-related plasma sheet motions as determined from differential timing of plasma changes at the ISEE satellites, J. Geophys. Res., 86, 3459,1981 . 
Foster, J. L., D. H. Falrfield, K. W. Ogllvie, and T. J. Rosenberg, Relationship of interplanetary parameters and occurrence of magnetospheric substorms, J. Geophys. Res., 76, 6967, 1971 .

Frandsen, A. M. A., B. V. Connor, J. van Amersfoort, and E. J. Smith, The ISEE-C vector hellum magnetometer, IEEE Trans. Geosc1. Electr., GE-16, 195, 1978.

Fuseller, S. A., W. C. Feldman, S. J. Bame, E.J. Smith, and F. L.Scarf, Heat flux observations and the location of the transition region boundary of Glacobini-zinner, Geophys. Res. Lett., 13, 247, 1986.

Gosling, J.T., J.R. Asbridge, S. J.Bame, M. F. Thomsen, and R. D. Zwickl, Large amplitude, low frequency plasma fluctuations at Comet Glacob1n1-Z1nner, Geophys. Res. Lett., 13, 267, 1986.

Hones, E. W. Jr., Transient phenomena in the magnetota1l and thelr relation to substorms, Space Sclence Reviews, 23 393, 1979.

Hones, E. W., Jr., J. Blrn, S. J. Bame, G. Paschmann, and C. T. Russe11, On the three-dimensional magnet1c structure of the plasmold created in the magnetotall at substorm onset, Geophys. Res. Lett., 9, 203, 1982.

Hones, E. W., Jr., J. Birn, D. N. Baker, S. J. Bame, W. C. Feldman, D. J. McConas, and R. D. Zwick1, Detalled examination of a plasmold in the distant magnetotall with ISEE-3 Geophys. Res. Lett. , 11, 1046, 1984 .

Huebner, W. F., Cometary coma, G. H. F. Dickerson et al. editors, Molecular Astrophys1cs, D. Reldel Pub. Co., 311, 1985.

Intriligator, D. S., J. H. Wolfe, and J. D. Mihalov, The Ploneer Venus Orbiter plasma analyzer experiment, IEEE Trans. on Geosclence and Remote Sensing, GE-19, $1,39,1980$.

Intriligator, D. S., and F. L. Scarf, Wave-particle Interactions in the Venus wake and tall, J. Geophys. Res., 89, 47, 1984.

Ip, W. H., and W. I. Axford, Theorles of physlcal processes in the cometary comae and Ion talls, in Comets, edited by L. L. Wilkening, University of Artzona Pres. Tucson, 1982.

Jones, D. E., E. J. Smith, J.A. Slavin, B. T. Tsurutan1, G. L. Siscoe, and D. A. Mendis, The bow wave of Comet Glacobini-zinner: ICE magnetic field observations, Geophys. Res. Lett., 13, 243, 1986.

Knoll, R., G. Epstein, S. Hoang, G. Huntzinger, J. L. Steinberg, J. Fainberg, F. Grena, S. R. Mosier, and R. G. Stone, The 
3-dImenstonal radio mapping experiment (SBH) on ISEE-C, IEEE Trans. Geosc1. Electr., GE-16, 199, 1978.

Luhmann, J. G., C. T. Russell, J. R. Spreiter and S. S. Stahara, Evidence for mass-loading of the Venus magnetosheath Adv. Space Res., 5(4), 307, 1985 .

Lu1, A. T. Y., C. I. Meng, and S. I. Akasofu, Wavy nature of the magnetotail neutral sheet, Geophys. Res. Lett., 5, 279, 1978.

Lu1, A. T. Y., Characteristics of the cross-tall current in the Earth's magnetotail, Magnetospheric Currents, Geophysical Monograph, Vol. 28, 158, 1983.

McComas, D. J. and C. T. Russe11, The near Earth current sheet: ISEE-1 and -2 case studies, Proc. Conf. Achlevements of the IMS, ESA, SP-217, 205, 1984 .

McComas, D.J., H. E. Spence, C. T. Russe11, and M. A. Saunders, The average magnetic fleld draping and consistent plasma properties of the Venus magnetotall, J.Geophys. Res., 91, 7939, 1986a.

McComas, D. J., H. E. Spence, and C. T. Russell, The average configuration of the Induced Venus magnetota11, accepted to Chapman Conference Proceedings on Magnetota11 Physics, $1986 \mathrm{~b}$.

McPherron, R. L., C. T. Russe11, and M. P. Aubry, Satell1te studles of magnetospheric substorms on August 15, 1969, 9. Phenomenologlcal model for substorms, J. Geophys. Res.s 78, $3131,1973$.

Mend1s, D. A., and H. L. F. Houp1s, The cometary atmosphere and 1 ts Interaction with the solar wind, Rev. Geophys., 20, 885, 1982.

Mendis, D. A., E. J. Sm1th, B. T. Tsurutan1, J. A. Slavin, D. E. Jones, and G. L. Siscoe, Comet-solar wind Interaction: Dynamical length scales and models, Geophys. Res. Lett., 13, 239, 1986.

Meyer-Vernet, N., P. Couturier, S. Hoang, C. Perche, J. L. Stelnberg, J. Fainberg, and C. Meetre, Plasma diagnosis from thermal nolse and $11 m i t s$ on dust flux or mass in comet P/G1acobini-Zinner, Science, 232, 370, 1986a.

Meyer-Vernet, N., P. Couturler, S. Hoang, C. Perche, and J. L. Stelnberg, Physical parameters for hot and cold electron populations in Comet Glacobini-zinner with the ICE radio experiment, Geophys. Res. Lett., 13, 279, 1986b.

Meyer-Vernet, N., M. A. Strauss, J. L. Stelnberg, H. Spinrad, and P. J. McCarthy, Comet P/Giacoblni-Zinner electron and $\mathrm{H}_{2} \mathrm{O}^{+}$ 
column densities from ICE and ground-based observations, submitted to Astronomical Journal, $1986 \mathrm{c}$.

Mihalov, J.D. and A. Barnes, Evidence for the acceleration of Ionospheric $0^{+}$in the magnetosheath of Venus, Geophys. Res. Lett., 8, 1277, 1981.

Mihalov, J. D., and A. Barnes, The distant interplanetary wake of Venus: plasma observations of Ploneer Venus, J. Geophys. Res., 87, 9045,1982 .

Moses, J. J., N. U. Crooker, D. J. Gorney, and G. L. Siscoe, High-latitude convection on open and closed field lines for large IMF $B_{y}$, submitted to the Journal of Geophyical Research, 1985.

Ogllvie, K. W., M. A. Coplan, P. Bochsler, and J. Gelss, Ion composition results during the International Cometary Explorer encounter with Giacobin1-Z1nner, Sclence, 232, 374, 1986.

Russell, C. T., The solar wind and magnetospheric dynamics, correlated tnterplanetary and magnetospherlc observations, edited by D. E. Page, 3, D. Rtede1, Hingham, Mass., 1974 .

Russe11, C. T., The magnetosphere of Venus: evidence for a boundary layer and a magnetota11, Geophys. Res. Lett., 3, 589, 1976.

Russell, C. T., R. C. Elphic, J.G. Luhmann, and J. A. Slavin, on the search for an Intrinsic magnetic fleld at Venus, Proc. Lunar Planet Conf. 11th, 1896, 1980a.

Russe11, C. T., R. C. Snare, J. D. Means, and R. C. Elphic, Ploneer Venus Orbiter Fluxgate Magnetometer, IEEE Trans. GE-18, 32, $1980 \mathrm{~b}$.

Russe11, C. T., J. G. Luhmann, R. C. Elphic, and F. L. Scarf, The distant bow shock and magnetotall of Venus: magnetic fleld and plasma wave observations, Geophys. Res. Lett., 즈, 843, 1981 .

Russe11, C. T., M. A. Saunders, and J. G. Luhmann, Mass-loading and the formation of the Venus tall, Adv. Space Res., $\underline{5}, 177-184$, 1985.

Saunders, M. A., and C. T. Russe11, Average dimension and magnetic structure of the distant Venus magnetotall, in press $\mathrm{J}$. Geophys. Res., 1986.

Saunders, M. A., C. T. Russell, I. G. Luhmann, Interactions with planetary lonospheres and atmospheres: a review, submitted to Proc. of Comparative Study of Magnetospheric Systems, 1985. 
Schmidt, H. L. and R. Wegmann, MHD-calculations for cometary plasmas, Computer Physics Communications, 19, 309, 1980.

Schm1dt, H. U., and R. Wegmann, Plasma flow and magnetlc flelds in comets, in Comets, (ed. L. L. Wilkening), University of Arizona Press; Tucson, Arizona; p. 538, 1982.

Sibeck, D. G., G. L. Siscoe, J. A. Slavin, E. J. Smith, B. T. Tsurutani, and R. P. Lepping, The distant magnetotail's response to a strong interpianetary magnetic fleld by: twisting, flattening, and fleld line bending, J. Geophys. Res., 90, 4011,1985 .

S1scoe, G. L., Solar system magnetohydrodynamics, Proceedings of the 1982 Boston College Theory Institute in Solar-Terrestrial Physics, R. L. Carovillano and J. M. Forbes, Ed Itors, D. ReIdel Pub. Co., Dordrecht-Holland, 198 ?.

Siscoe, G. L., J. A. Slavin, E. J. Smith, B. T. Tsurutani, D. E. Jones, and D. A. Mend1s, Statics and dynamics of Glacobini-Zinner magnetic tall, Geophys. Res. Lett., 13, 287 , 1986.

Slavin, J.A., E. J. Smith, B. T. Tsurutani, G. L. Siscoe, D. E. Jones, and D. A. Mend1s, Glacob1n1-Z1nner magnetoLa11: ICE magnetic field observations, Geophys. Res. Lett., 13, 283, $1986 \mathrm{a}$.

Slavin, J. A., B. A. Goldberg, E. J. Smith, D. J. McComas, S. J. Bame, M A. Strauss, and H. Splnrad, The structure of a cometary type I tal1: ground-based and ICE observations of P/Giacobini-ZInner, submitted to Geophys. Res. Lett., 1986b.

Slavin, J. A., E. J. Smith, D. S. Intriligator, A comparative study of distant magnetotail structure at Venus and Earth, Geophys. Res. Lett., 11, 1074, 1984 .

Slavin, J. A., D. S. Intriligator, and E. J. Smith, Initial PVo Observations of Magnetic Field Draping at Venus Due to Mass Loading: Implications for Magnetotall Formation at Venus and Comets, Submitted to J. Geophys. Res., 1985.

Smith, E. J., B. T. Tsurutanl, J. A. Slavin, D. E. Jones, G. L. Siscoe, and D. A. Mendis, ICE encounter with Giacobini-Zinner: Magnetic fleld observations, Sclence, 232, $382,1986$.

Speiser, T. W., Particle trajectories in model current sheets 1. Analytic solutions, J. Geophys. Res., 70, 4219, 1965. 
Spretter, J. R. and S. S. Stahara, Solar wind flow past Venus: Theory and comparisons, J.Geophys. Res., 85, 7715, 1980 .

Strauss, M. A., P. J. McCarthy, and H. Spinrad, Optical spectrophotometry of Comet P/Giacobini-Zinner and emission profiles of $\mathrm{H}_{2} \mathrm{O}^{+}$, Geophys. Res. Lett., 13, 389, 1986.

Tsurutant, B. T., and E. J. Smith, Strong hydromagnetic turbulence associated with Comet Giacobini-Zinner, Geophys. Res. Lett., 13, $259,1986 a$.

Tsurutant, B. T., and E. J. Sm1th, Hydromagnetic waves and instabilities associated with cometary ion pick-up: ICE observations, Geophys. Res. Lett., 13, 263, 1986b.

Voigt, G. -H. and R. Hilmer, The influence of the IMF $B_{Y}$ component on the earth's magneto-hydrostatic magnetotail, Accepted to Chapman Conference Proceedings on Magnetotail Physics, 1986.

Zwick1, R. D., D. N. Baker, S. J. Bame, W. C. Feldman, S. A. Fuselier, W. F. Huebner, D. J. McComas, and D. T. Young, Three component plasma electron distributions in the intermediate lonized coma of Comet Giacobini-zinner, Geophys. Res. Lett., 13, 401, 1986. 